\title{
Arylation of ortho-hydroxyarylenaminones by sulfonium salts and arenesulfonyl chlorides: An access to isoflavones.
}

Satenik Mkrtchyan ${ }^{a *}$, Viktor O. laroshenko ${ }^{a, b, c *}$

aLaboratory of Homogeneous Catalysis and Molecular Design at the Centre of Molecular and Macromolecular Studies, Polish Academy of Sciences, Sienkiewicza 112, PL-90-363 Łodź, Poland. E-mail: iva108@gmail.com, viktori@cbmm.lodz.pl

bDipartimento di Chimica e Biologia "A. Zambelli" Università di Salerno Via Giovanni Paolo II, 84084, Fisciano (SA), Italy.

'Department of Chemistry, University of Helsinki, A.I. Virtasen aukio 1, 00014 Helsinki, Finland. 


\section{Table of Contents}

(A) Experimental Section $\quad$ S3

(A-1) Scope of reagents used

(A-2) Reaction condition screening for arylation of ortho-hydroxyarylenaminones

(B) Copies ${ }^{1} \mathrm{H}$ and ${ }^{13} \mathrm{C}$ NMR spectra 


\section{(A) Experimental Section.}

\section{A-1. Scope of the reagents used.}

Scope of ortho-hydroxyarylenaminones 1:

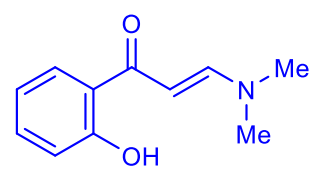

1a<smiles>Cc1ccc(O)c(C(=O)/C=C/N(C)C)c1</smiles>

1b

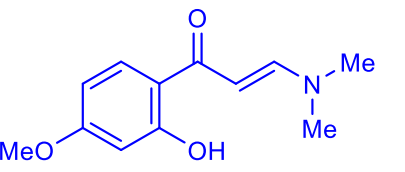

$1 \mathrm{c}$

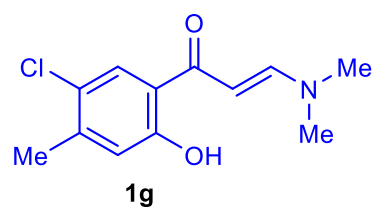

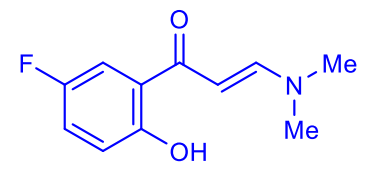

1d

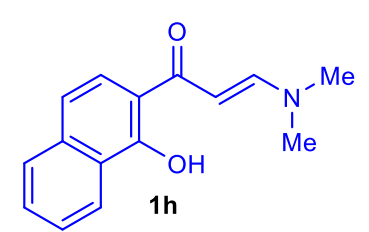

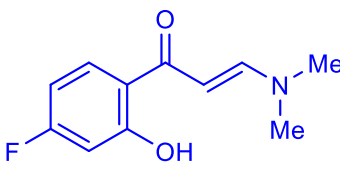

$1 e$

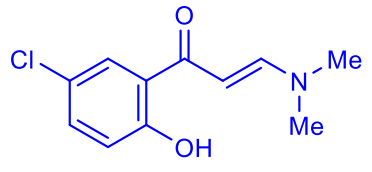

$1 f$

Scheme 1. List of ortho-hydroxyarylenaminones 1 used for preparation of 3-arylchromones. 
Scope of O-substituted ortho-hydroxyarylenaminones 6:

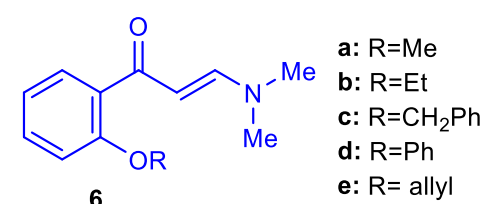

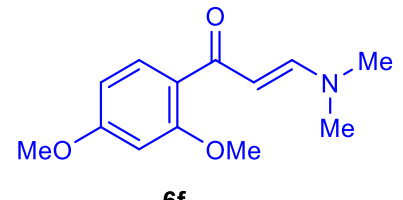

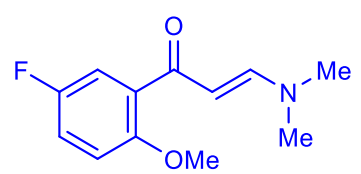

$6 \mathrm{~g}$

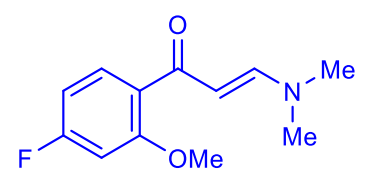

$6 \mathrm{~h}$

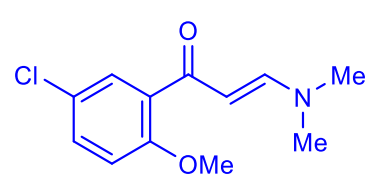

$6 \mathbf{i}$

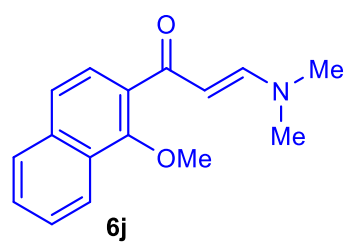

Scheme 2. List of O-substituted ortho-hydroxyarylenaminones 6 used for preparation of 3-arylchromones.

\section{Scope of dimethyl(aryl)sulfoniums 2:}
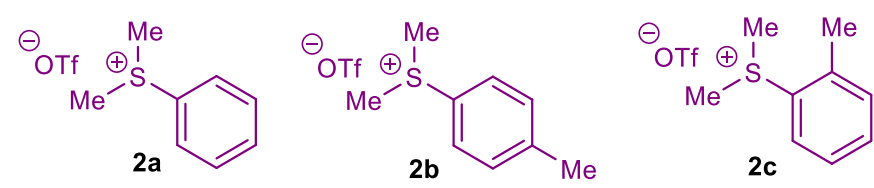

2c
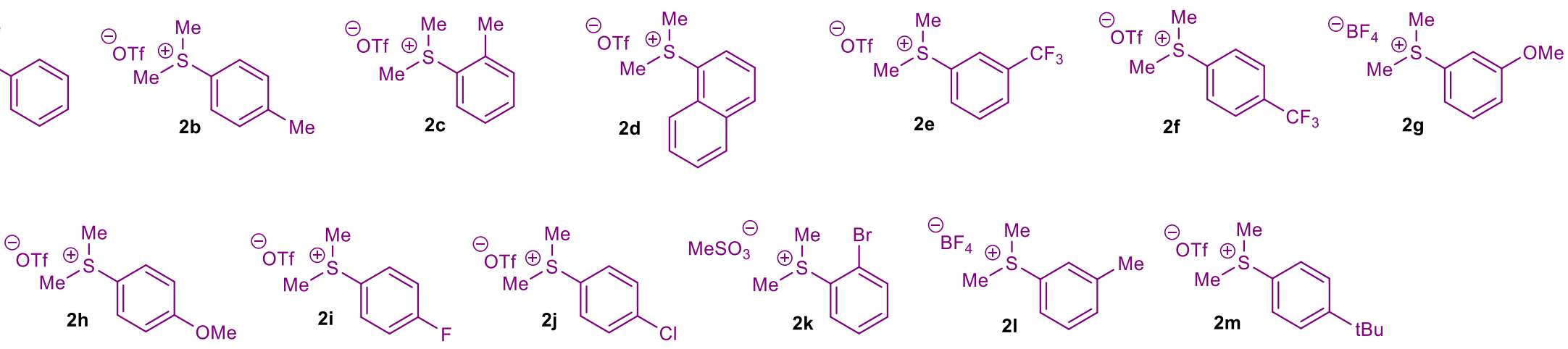

Scheme 3. List of dimethyl(aryl)sulfonium salts $\mathbf{2}$ used for preparation of 3-arylchromones. 
Scope of triarylsulfoniums 3 :
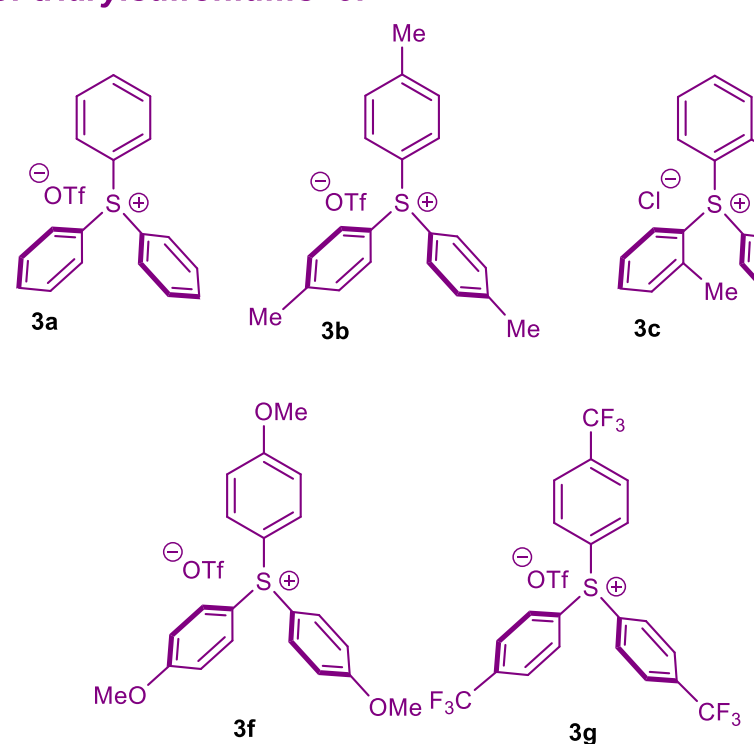
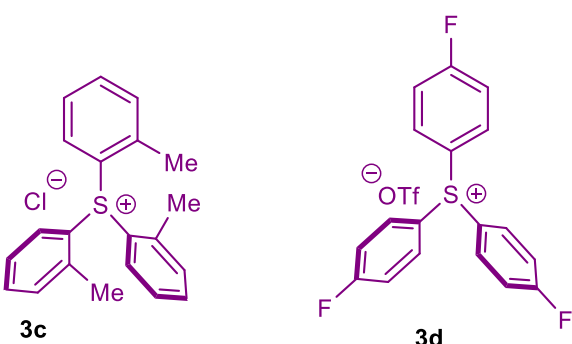

3d
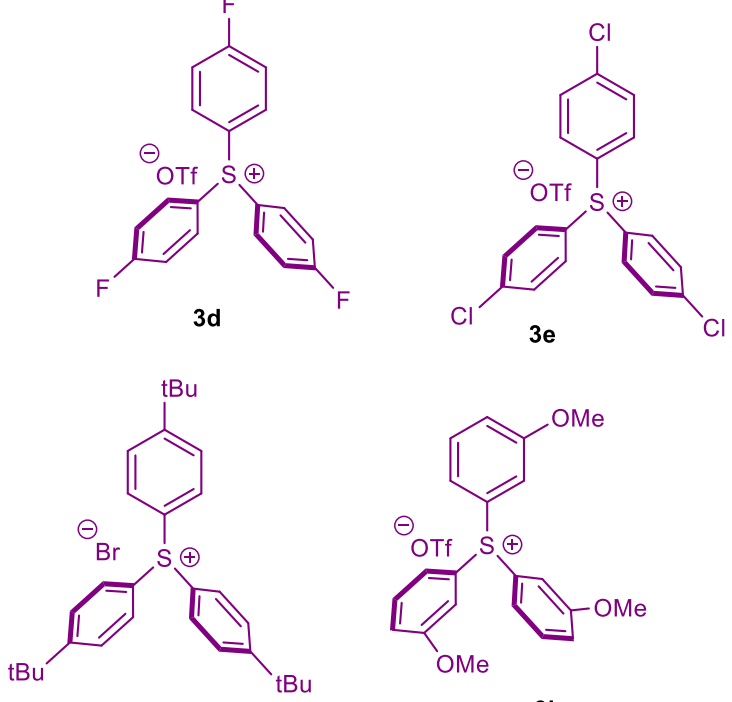

$3 \mathrm{~h}$

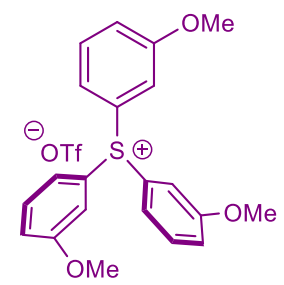

$3 \mathbf{i}$

Scheme 4. List of triarylsulfonium salts $\mathbf{3}$ used for preparation of 3-arylchromones.

Scope of arenesulfonyl chlorides 4:<smiles>O=S(=O)(Cl)c1ccccc1</smiles><smiles>Cc1ccc(S(=O)(=O)Cl)cc1</smiles><smiles>O=S(=O)(Cl)c1cccc2ccccc12</smiles>
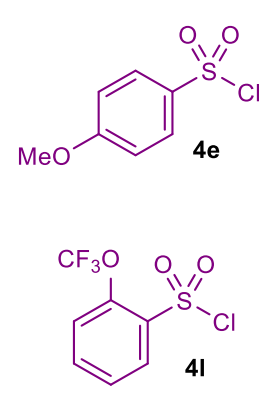
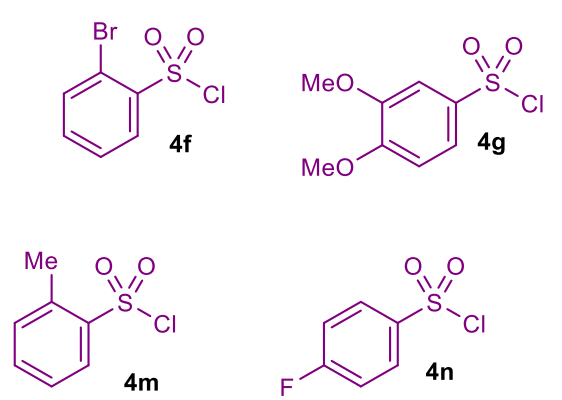

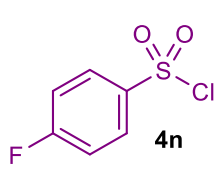

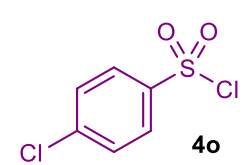
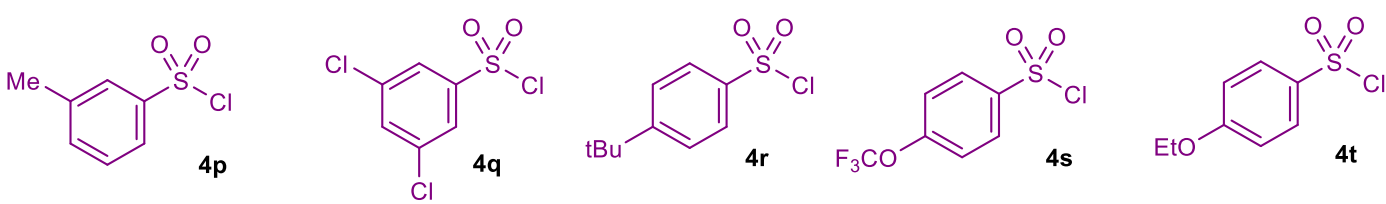

Scheme 5. List of arenesulfonyl chlorides $\mathbf{4}$ used for preparation of 3-arylchromones. 


\section{A-2. Reaction condition screening for arylation of ortho-hydroxyarylenaminones.}

Table S1. Optimization of the reaction conditions.

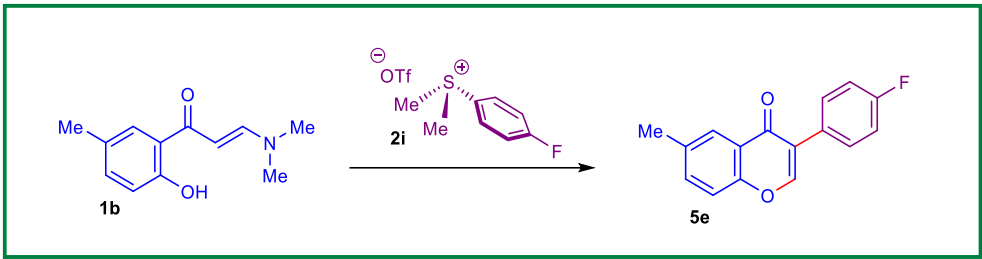

\begin{tabular}{|c|c|c|c|}
\hline \multicolumn{4}{|c|}{ Reaction $(\mathbf{a})^{b}$} \\
\hline entry & reagent (equiv)/base & Solvent/temperature/time & yield $(\%) 5 e^{a}$ \\
\hline 2 & sulfonium salt $(1.5) / \mathrm{Cs}_{2} \mathrm{CO}_{3}(1.5)$ & $\mathrm{CH}_{3} \mathrm{CN} / 120^{\circ} \mathrm{C} / 10 \mathrm{~h}$ & $19^{d}$ \\
\hline 4 & sulfonium salt $(1.5) / \mathrm{K}_{2} \mathrm{CO}_{3}(1.5)$ & $\mathrm{CH}_{3} \mathrm{CN} / 0^{\circ} \mathrm{C}$ to r.t. to $120^{\circ} \mathrm{C} / 0.5 \mathrm{~h}+1 \mathrm{~h}+12 \mathrm{~h}$ & $20^{d}$ \\
\hline 5 & sulfonium salt (1.5)/K $\mathrm{K}_{2} \mathrm{CO}_{3}(1.5)$ & DMSO $/ 0^{\circ} \mathrm{C}$ to r.t. to $90^{\circ} \mathrm{C} / 0.5 \mathrm{~h}+1 \mathrm{~h}+7 \mathrm{~h}$ & $39 \mathrm{~d}, \mathrm{e}$ \\
\hline 6 & sulfonium salt (1.5)/ $/ \mathrm{Cs}_{2} \mathrm{CO}_{3}(1.5)$ & DMSO $/ 0^{\circ} \mathrm{C}$ to r.t. to $90^{\circ} \mathrm{C} / 0.5 \mathrm{~h}+1 \mathrm{~h}+7 \mathrm{~h}$ & $42^{\mathrm{d}, \mathrm{e}}$ \\
\hline 9 & sulfonium salt (1.3)/ $\mathrm{Cs}_{2} \mathrm{CO}_{3}(1.5)$ & $\mathrm{DMF} / 0^{\circ} \mathrm{C}$ to r.t. to $90^{\circ} \mathrm{C} / 0.5 \mathrm{~h}+1 \mathrm{~h}+5 \mathrm{~h}$ & 89 \\
\hline 10 & sulfonium salt $(1.3) / \mathrm{Cs}_{2} \mathrm{CO}_{3}(1.5)$ & $\mathrm{DMF} / 0^{\circ} \mathrm{C}$ to r.t. to $110^{\circ} \mathrm{C} / 0.5 \mathrm{~h}+1 \mathrm{~h}+5 \mathrm{~h}$ & 86 \\
\hline 11 & sulfonium salt $(1.3) / \mathrm{CS}_{2} \mathrm{CO}_{3}(1.5)$ & $\mathrm{DMF} / 0^{\circ} \mathrm{C}$ to r.t. to $120^{\circ} \mathrm{C} / 0.5 \mathrm{~h}+1 \mathrm{~h}+5 \mathrm{~h}$ & 87 \\
\hline 12 & sulfonium salt (1.3)/ $/ \mathrm{Cs}_{2} \mathrm{CO}_{3}(1.5)$ & $\mathrm{DMF} / 0^{\circ} \mathrm{C}$ to r.t. to $90^{\circ} \mathrm{C} / 0.5 \mathrm{~h}+1 \mathrm{~h}+3 \mathrm{~h}$ & 76 \\
\hline 13 & sulfonium salt $(1.3) / \mathrm{K}_{2} \mathrm{CO}_{3}(1.5)$ & $\mathrm{DMF} / 0^{\circ} \mathrm{C}$ to r.t. to $90^{\circ} \mathrm{C} / 0.5 \mathrm{~h}+1 \mathrm{~h}+5 \mathrm{~h}$ & 68 \\
\hline 14 & sulfonium salt $(1.3) / \mathrm{K}_{2} \mathrm{CO}_{3}(1.5) / \mathrm{TEMPO}(2.0)$ & $\mathrm{DMF} / 0^{\circ} \mathrm{C}$ to r.t. to $90^{\circ} \mathrm{C} / 0.5 \mathrm{~h}+1 \mathrm{~h}+5 \mathrm{~h}$ & 81 \\
\hline
\end{tabular}

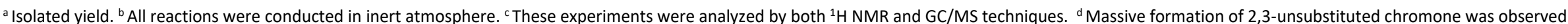
e Some amount of starting ortho-hydroxyarylenaminone was recovered.

Table S2. Optimization of the reaction conditions. 


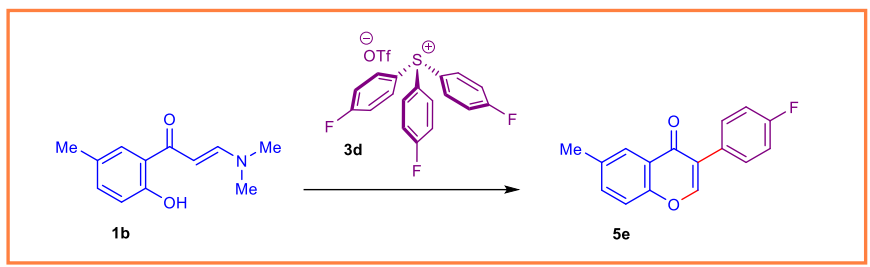

\begin{tabular}{|c|c|c|c|c|}
\hline \multicolumn{5}{|c|}{ Reaction $(\mathbf{b})^{\mathbf{b}}$} \\
\hline entry & reagent (equiv)/catalysts (equiv)/base (equiv) & light source & solvent/time & yield $(\%) \mathbf{5 e}^{\mathrm{a}}$ \\
\hline 1 & sulfonium salt (1.5)/fac-Ir(ppy) $)_{3} \mathrm{I}(0.01) / \mathrm{Na}_{2} \mathrm{CO}_{3}(1.1)$ & Blue LED & $\mathrm{MeOH} / 15 \mathrm{~h}$ & 31 \\
\hline 2 & sulfonium salt (1.5)/fac-Ir(ppy) ${ }_{3} \mathrm{I}(0.01) / \mathrm{NaOAc}(1.8)$ & Blue LED & Acetone/10h & 40 \\
\hline 3 & sulfonium salt (1.5)/fac- $\operatorname{Ir}(\mathrm{ppy})_{3} \mathrm{I}(0.01) / \mathrm{NaOAc}(1.8)$ & Blue LED & $\mathrm{MeOH} / 15 \mathrm{~h}$ & 11 \\
\hline 4 & sulfonium salt (1.5)/fac-Ir(ppy) ${ }_{3} \mathrm{I}(0.01) / \mathrm{NaOAc}(1.8)$ & Blue LED & $\mathrm{CH}_{3} \mathrm{CN} / 15 \mathrm{~h}$ & 71 \\
\hline 5 & sulfonium salt (1.5)/fac- $\operatorname{Ir}(\mathrm{ppy})_{3} \mathrm{I}(0.01) / \mathrm{NaOAc}(1.8)$ & Blue LED & DMSO /10h & 83 \\
\hline 6 & sulfonium salt (1.5)/fac-Ir(ppy) ${ }_{3}$ I (0.01)/NaOAc (1.8) & Blue LED & DMSO /4h & 84 \\
\hline 7 & 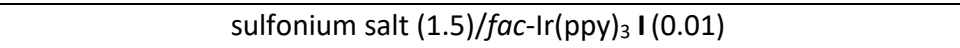 & Blue LED & DMSO /4h & 55 \\
\hline 8 & sulfonium salt $(1.5) / \operatorname{Ir}\left[\mathrm{dF}\left(\mathrm{CF}_{3}\right) \mathrm{ppy}\right]_{2}(\mathrm{dtbbpy}) \mathrm{PF}_{6} \mathrm{II}(0.01)$ & Blue LED & DMSO /4h & 67 \\
\hline 9 & sulfonium salt (1.5)/Ir[dF(CF $)$ ppy $]_{2}(\mathrm{dtbbpy}) \mathrm{PF}_{6} \mathrm{II}(0.01) / \mathrm{Na}_{2} \mathrm{CO}_{3}(1.5)$ & Blue LED & DMSO /4h & 60 \\
\hline 10 & sulfonium salt (1.5)/Ir[dF(CF 3 )ppy $]_{2}(\mathrm{dtbbpy}) \mathrm{PF}_{6} \mathrm{II}(0.01) / \mathrm{NaOAc}(1.8)$ & Blue LED & DMSO /4h & 89 \\
\hline 11 & sulfonium salt (1.5)/Ru(bpy) ${ }_{3} \mathrm{Cl}_{2} * 6 \mathrm{H}_{2} \mathrm{O} \mathrm{III}(0.02)$ & Blue LED & DMSO/12h & 41 \\
\hline 12 & sulfonium salt (1.5)/Ru(bpy) ${ }_{3} \mathrm{Cl}_{2} * 6 \mathrm{H}_{2} \mathrm{O}$ III $(0.02) / \mathrm{NaOAc}$ (1.8) & Blue LED & $\mathrm{MeOH} / 12 \mathrm{~h}$ & 74 \\
\hline 13 & sulfonium salt (1.5)/Ru(bpy) ${ }_{3} \mathrm{Cl}_{2}{ }^{*} 6 \mathrm{H}_{2} \mathrm{O}$ III $(0.02) / \mathrm{NaOAc}(1.8)$ & Blue LED & $\mathrm{CH}_{3} \mathrm{CN} / 12 \mathrm{~h}$ & 88 \\
\hline$\underline{14}$ & sulfonium salt (1.4)/Ru(bpy) ${ }_{3} \underline{C l}_{2}{ }^{*} 6 \mathrm{H}_{2} \underline{O}$ III (0.02)/NaOAC (1.8) & Blue LED & $\underline{\mathrm{CH}_{3}} \underline{\mathrm{CN} / 3 \mathrm{~h}}$ & $\underline{92}$ \\
\hline 15 & sulfonium salt (1.4)/Ru(bpy) ${ }_{3} \mathrm{Cl}_{2}{ }^{*} 6 \mathrm{H}_{2} \mathrm{O}$ III $(0.02) / \mathrm{NaOAc}$ (1.8) & Blue LED & $\mathrm{CH}_{3} \mathrm{CN} / 3 \mathrm{~h}$ & 80 \\
\hline 16 & sulfonium salt (1.4)/Ru(bpy) ${ }_{3} \mathrm{Cl}_{2} * 6 \mathrm{H}_{2} \mathrm{O}$ III $(0.02) / \mathrm{Na}_{2} \mathrm{CO}_{3}$ (1.8) & Blue LED & $\mathrm{CH}_{3} \mathrm{CN} / 3 \mathrm{~h}$ & 43 \\
\hline 17 & sulfonium salt (1.4)/Ru(bpy) ${ }_{3} \mathrm{Cl}_{2} * 6 \mathrm{H}_{2} \mathrm{O}$ III $(0.02) / \mathrm{NaOAc}(1.8) / \mathrm{TEMPO}(1.8)$ & Blue LED & $\mathrm{CH}_{3} \mathrm{CN} / 3 \mathrm{~h}$ & $0^{c}$ \\
\hline 18 & sulfonium salt (1.4)/Ru(bpy) ${ }_{3} \mathrm{Cl}_{2}{ }^{*} 6 \mathrm{H}_{2} \mathrm{O}$ III $(0.02) / \mathrm{NaOAc}(1.8)$ & NO & $\mathrm{CH}_{3} \mathrm{CN} / 3 \mathrm{~h}$ & $0^{c}$ \\
\hline 19 & sulfonium salt (1.4)/ $\mathrm{NaOAc}(1.8)$ & Blue LED & $\mathrm{CH}_{3} \mathrm{CN} / 3 \mathrm{~h}$ & $0^{c}$ \\
\hline 20 & sulfonium salt (1.4)/Ru(bpm) ${ }_{3} \mathrm{Cl}_{2}{ }^{*} 6 \mathrm{H}_{2} \mathrm{O}$ IV (0.02)/NaOAc (1.8) & Blue LED & $\mathrm{CH}_{3} \mathrm{CN} / 4 \mathrm{~h}$ & 71 \\
\hline 21 & sulfonium salt (1.4)/Ru(bpz) ${ }_{3} \mathrm{Cl}_{2} * 6 \mathrm{H}_{2} \mathrm{O} \mathrm{V}(0.02) / \mathrm{NaOAc}(1.8)$ & Blue LED & $\mathrm{CH}_{3} \mathrm{CN} / 4 \mathrm{~h}$ & 77 \\
\hline 22 & sulfonium salt (1.4)/[Cu(dpp) $\left.{ }_{2}\right] \mathrm{PF}_{6} \mathrm{VI}(0.02) / \mathrm{NaOAc}(1.8)$ & Green LED & $\mathrm{CH}_{3} \mathrm{CN} / 12 \mathrm{~h}$ & 34 \\
\hline 23 & sulfonium salt $(1.4) /\left[\mathrm{Cu}(\mathrm{dpp})_{2}\right] \mathrm{PF}_{6} \mathrm{VI}(0.02) / \mathrm{Na}_{2} \mathrm{CO}_{3}(1.8)$ & Green LED & DMSO/12h & 48 \\
\hline 24 & sulfonium salt $(1.4) /\left[\mathrm{Cu}(\text { dap })_{2}\right] \mathrm{PF}_{6} \mathrm{VII}(0.02) / \mathrm{Na}_{2} \mathrm{CO}_{3}(1.8)$ & Green LED & $\mathrm{CH}_{3} \mathrm{CN} / 12 \mathrm{~h}$ & 30 \\
\hline 25 & sulfonium salt (1.4)/[Cu(dap) $\left.{ }_{2}\right] \mathrm{PF}_{6} \mathrm{VII}(0.02) / \mathrm{K}_{2} \mathrm{HPO}_{4}(2)$ & Green LED & DMSO/12h & 14 \\
\hline 26 & sulfonium salt (1.4)/Eosin Y VIII (0.03)/NaOAc (1.8) & Green LED & DMSO/12h & 31 \\
\hline 27 & sulfonium salt (1.4)/Eosin Y VIII (0.03) & Green LED & $\mathrm{MeOH} / 12 \mathrm{~h}$ & 37 \\
\hline
\end{tabular}

${ }^{\mathrm{a}}$ Isolated yield. ${ }^{\mathrm{b}}$ All reactions were conducted at room temperature in inert atmosphere. ${ }^{\mathrm{c}}$ These experiments were analyzed by both ${ }^{1} \mathrm{H}$ NMR and GC/MS techniques. 
Table S3. Optimization of the reaction conditions.

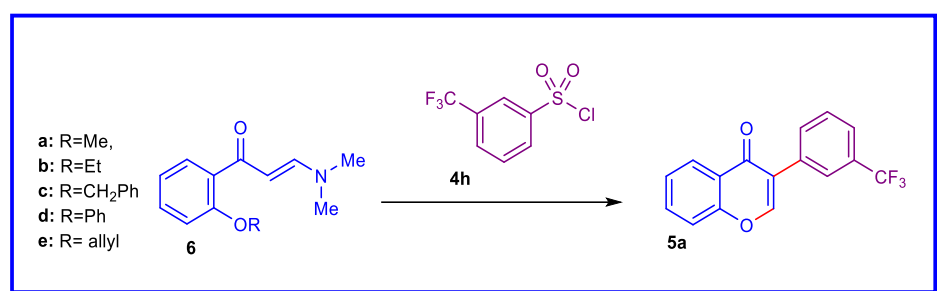

\begin{tabular}{|c|c|c|c|c|}
\hline \multicolumn{5}{|c|}{ Reaction (c) ${ }^{\mathbf{b}}$} \\
\hline entry & reagent (equiv)/catalysts (equiv)/base (equiv) & light source & solvent/time & yield $(\%) \mathbf{5} a^{a}$ \\
\hline 1 & arenesulfonyl chloride (2.8)/ $\mathrm{Ru}(\mathrm{bpy})_{3} \mathrm{Cl}_{2} * 6 \mathrm{H}_{2} \mathrm{O} \mathrm{III}(0.02) / \mathrm{K}_{2} \mathrm{HPO}_{4}(3)$ & Blue LED & $\mathrm{CH}_{3} \mathrm{CN} / 12 \mathrm{~h}$ & $39^{d}$ \\
\hline 2 & arenesulfonyl chloride (2.8)/ $\mathrm{Ru}(\mathrm{bpy})_{3} \mathrm{Cl}_{2} * 6 \mathrm{H}_{2} \mathrm{O} \mathrm{III}(0.02) / \mathrm{K}_{2} \mathrm{HPO}_{4}(3)$ & Haushold Light (26W) & $\mathrm{CH}_{3} \mathrm{CN} / 12 \mathrm{~h}$ & $28^{d}$ \\
\hline 3 & arenesulfonyl chloride (2.8)/ $\mathrm{Ru}(\mathrm{bpy})_{3} \mathrm{Cl}_{2}{ }^{*} 6 \mathrm{H}_{2} \mathrm{O} \mathrm{III}(0.02) / \mathrm{iPr}_{2} \mathrm{NEt}$ (4) & Blue LED & $\mathrm{CH}_{3} \mathrm{CN} / 12 \mathrm{~h}$ & $0^{d}$ \\
\hline 4 & arenesulfonyl chloride (2.8)/Ru(bpy) ${ }_{3} \mathrm{Cl}_{2} * 6 \mathrm{H}_{2} \mathrm{O}$ III $(0.02) / 2,4,6$-collidine (4) & Blue LED & $\mathrm{CH}_{3} \mathrm{CN} / 12 \mathrm{~h}$ & $0^{d}$ \\
\hline 5 & arenesulfonyl chloride (2.8)/Ir[dF(CF $)$ ppy $]_{2}(\mathrm{dtbbpy}) \mathrm{PF}_{6} \mathrm{II}(0.01) / \mathrm{Na}_{2} \mathrm{CO}_{3}(3)$ & Blue LED & EtOH:acetone $(1: 2) / 12 \mathrm{~h}$ & $11^{\mathrm{d}}$ \\
\hline 6 & arenesulfonyl chloride $(2.8) / \operatorname{Ir}\left[\mathrm{dF}\left(\mathrm{CF}_{3}\right) \text { ppy }\right]_{2}(\mathrm{dtbbpy}) \mathrm{PF}_{6} \mathrm{II}(0.01) / \mathrm{Na}_{2} \mathrm{CO}_{3}(3)$ & Blue LED & $\mathrm{CH}_{3} \mathrm{CN} / 12 \mathrm{~h}$ & $44^{d}$ \\
\hline 7 & arenesulfonyl chloride (2.8)/Ir[ $\left.\mathrm{dF}\left(\mathrm{CF}_{3}\right) \mathrm{ppy}\right]_{2}(\mathrm{dtbbpy}) \mathrm{PF}_{6} \mathrm{II}(0.01) / \mathrm{K}_{2} \mathrm{HPO}_{4}(3)$ & Blue LED & $\mathrm{CH}_{3} \mathrm{CN}: \mathrm{H}_{2} \mathrm{O}(5: 1) / 12 \mathrm{~h}$ & $12^{\mathrm{d}}$ \\
\hline 8 & arenesulfonyl chloride (2.8)//r[dF(CF $)$ ppy $]_{2}(\mathrm{dtbbpy}) \mathrm{PF}_{6} \mathrm{II}(0.01) / \mathrm{K}_{2} \mathrm{HPO}_{4}(3)$ & Blue LED & $\mathrm{CH}_{3} \mathrm{CN} / 12 \mathrm{~h}$ & $20^{d}$ \\
\hline 9 & arenesulfonyl chloride (2.8)/Eosin $\mathrm{Y}$ VIII $(0.03) / \mathrm{K}_{2} \mathrm{HPO}_{4}(3)$ & Blue LED & $\mathrm{CH}_{3} \mathrm{CN} / 12 \mathrm{~h}$ & $25^{d}$ \\
\hline 10 & arenesulfonyl chloride (2.8)/Eosin Y VIII $(0.03) / \mathrm{K}_{2} \mathrm{HPO}_{4}(3)$ & Blue LED & EtOH:Acetone $(1: 2) / 12 \mathrm{~h}$ & $21^{d}$ \\
\hline 11 & arenesulfonyl chloride (2.8)/Eosin $\mathrm{Y} \mathrm{VIII}(0.03) / \mathrm{K}_{2} \mathrm{HPO}_{4}(3)$ & Blue LED & $\mathrm{CH}_{3} \mathrm{CN}: \mathrm{H}_{2} \mathrm{O}(5: 1) / 12 \mathrm{~h}$ & $28^{d}$ \\
\hline 12 & arenesulfonyl chloride (2.8)/Eosin Y VIII $(0.03) / \mathrm{K}_{2} \mathrm{HPO}_{4}(3)$ & Blue LED & $\mathrm{CH}_{3} \mathrm{CN}: \mathrm{H}_{2} \mathrm{O}(5: 1) / 3 \mathrm{~h}$ & $17^{d}$ \\
\hline 13 & arenesulfonyl chloride (2.8)/Eosin Y VIII (0.03) & Blue LED & $\mathrm{CH}_{3} \mathrm{CN}: \mathrm{H}_{2} \mathrm{O}(5: 1) / 12 \mathrm{~h}$ & $4^{d}$ \\
\hline 14 & arenesulfonyl chloride $(2.2) / f a c-I r(p p y)_{3} I(0.01) / \mathrm{Na}_{2} \mathrm{CO}_{3}(2)$ & Blue LED & DMSO/12h & 23 \\
\hline 15 & arenesulfonyl chloride (2.2)/fac-Ir(ppy) ${ }_{3} \mathrm{I}(0.01) / \mathrm{Na}_{2} \mathrm{CO}_{3}(2)$ & Blue LED & $\mathrm{DMF} / 12 \mathrm{~h}$ & 67 \\
\hline 16 & arenesulfonyl chloride (2.2)/fac-Ir(ppy) ${ }_{3}$ I (0.01)/ $\mathrm{Na}_{2} \mathrm{CO}_{3}(2)$ & Blue LED & $\mathrm{CH}_{3} \mathrm{CN} / 12 \mathrm{~h}$ & 79 \\
\hline 17 & arenesulfonyl chloride $(2.2) / \operatorname{Ir}\left[\mathrm{dF}\left(\mathrm{CF}_{3}\right) \text { ppy }\right]_{2}(\mathrm{dtbbpy}) \mathrm{PF}_{6} \mathrm{II}(0.01) / \mathrm{Na}_{2} \mathrm{CO}_{3}(2)$ & Blue LED & $\mathrm{CH}_{3} \mathrm{CN} / 12 \mathrm{~h}$ & 83 \\
\hline 18 & arenesulfonyl chloride (2.2)/Ir[ $\left.\mathrm{dF}\left(\mathrm{CF}_{3}\right) \mathrm{ppy}\right]_{2}(\mathrm{dtbbpy}) \mathrm{PF}_{6} \mathrm{II}(0.01) / \mathrm{Na}_{2} \mathrm{CO}_{3}(2)$ & Blue LED & $\mathrm{CH}_{3} \mathrm{CN} / 12 \mathrm{~h}$ & $80^{f}$ \\
\hline 19 & arenesulfonyl chloride (2.2)/Ir[ $\left.\mathrm{dF}\left(\mathrm{CF}_{3}\right) \mathrm{ppy}\right]_{2}(\mathrm{dtbbpy}) \mathrm{PF}_{6} \mathrm{II}(0.01) / \mathrm{Na}_{2} \mathrm{CO}_{3}(2)$ & Blue LED & $\mathrm{CH}_{3} \mathrm{CN} / 12 \mathrm{~h}$ & $85^{f}$ \\
\hline 20 & arenesulfonyl chloride (2.2)/Ir $\left[\mathrm{dF}\left(\mathrm{CF}_{3}\right) \mathrm{ppy}\right]_{2}(\mathrm{dtbbpy}) \mathrm{PF}_{6} \mathrm{II}(0.01) / \mathrm{Na}_{2} \mathrm{CO}_{3}(2)$ & Blue LED & $\mathrm{CH}_{3} \mathrm{CN} / 12 \mathrm{~h}$ & $68^{g}$ \\
\hline 21 & arenesulfonyl chloride (2.2)/Ru(bpy) ${ }_{3} \mathrm{Cl}_{2} * 6 \mathrm{H}_{2} \mathrm{O} \mathrm{III}(0.02) / \mathrm{Na}_{2} \mathrm{CO}_{3}(2)$ & Blue LED & DMSO/12h & 33 \\
\hline 22 & arenesulfonyl chloride $(2.2) / \mathrm{Ru}(\mathrm{bpy}){ }_{3} \mathrm{Cl}_{2} * 6 \mathrm{H}_{2} \mathrm{O} \mathrm{III}(0.02) / \mathrm{Na}_{2} \mathrm{CO}_{3}(2)$ & Blue LED & $\mathrm{DMF} / 12 \mathrm{~h}$ & 65 \\
\hline 23 & arenesulfonyl chloride $(2.2) / \mathrm{Ru}(\mathrm{bpy}){ }_{3} \mathrm{Cl}_{2} * 6 \mathrm{H}_{2} \mathrm{O} \mathrm{III}(0.02) / \mathrm{Na}_{2} \mathrm{CO}_{3}$ (2) & Blue LED & $\mathrm{CH}_{3} \mathrm{CN} / 12 \mathrm{~h}$ & 91 \\
\hline 24 & arenesulfonyl chloride $(2.2) / \mathrm{Ru}(\mathrm{bpy})_{3} \mathrm{Cl}_{2} * 6 \mathrm{H}_{2} \mathrm{O} \mathrm{III}(0.02) / \mathrm{Na}_{2} \mathrm{CO}_{3}$ (2) & Blue LED & $\mathrm{CH}_{3} \mathrm{CN} / 4 \mathrm{~h}$ & 90 \\
\hline 25 & arenesulfonyl chloride (1.8)/Ru(bpy) ${ }_{3} \underline{C l}_{2}{ }_{2}{ }_{6} \mathrm{H}_{2} \mathrm{O}_{2} \mathrm{III}(0.02) / \mathrm{Na}_{2}{ }_{2} \mathrm{CO}_{3}(2)$ & Blue LED & $\underline{\mathrm{CH}_{3}} \underline{\mathrm{CN} / 4 h}$ & $\underline{90}$ \\
\hline 26 & arenesulfonyl chloride (1.8)/Ru(bpy) ${ }_{3} \underline{\mathrm{Cl}}_{2}{ }_{2}{ }_{6} \mathrm{H}_{2}{ }_{2} \mathrm{O} \mathrm{III}(0.02) / \mathrm{Na}_{2} \underline{\mathrm{CO}}_{3}{ }_{2}$ (2) & Blue LED & $\underline{\mathrm{CH}_{3}} \underline{\mathrm{CN} / 4 \mathrm{~h}}$ & $85^{e}$ \\
\hline 27 & arenesulfonyl chloride (1.8)/Ru(bpv) ${ }_{3} \mathrm{Cl}_{2}{ }_{2}{ }_{6} \mathrm{H}_{2} \mathrm{O}$ III $(0.02) / \mathrm{Na}_{2} \underline{\mathrm{CO}}_{3}$ (2) & Blue LED & $\underline{\mathrm{CH}_{3} \mathrm{CN} / 4 \mathrm{~h}}$ & $91^{f}$ \\
\hline 28 & arenesulfonyl chloride (1.8)/Ru(bpy) ${ }_{3} \underline{\mathrm{Cl}}_{2}{ }_{2}{ }_{6} \mathrm{H}_{2} \mathrm{H}_{2} \mathrm{OIII}(0.02) / \mathrm{Na}_{2} \underline{\mathrm{CO}}_{3}{ }_{2}$ (2) & Blue LED & $\underline{\mathrm{CH}_{3}} \underline{\mathrm{CN} / 4 \mathrm{~h}}$ & $61^{g}$ \\
\hline 29 & arenesulfonyl chloride (1.8)/Ru(bpy) ${ }_{3} \mathrm{Cl}_{2}{ }_{2}{ }_{6} \mathrm{H}_{2} \mathrm{O} \mathrm{III} \mathrm{(0.02)/ \textrm {Na } _ { 2 }} \underline{\mathrm{CO}}_{3}(2)$ & Blue LED & $\underline{\mathrm{CH}_{3}} \underline{\mathrm{CN} / 4 \mathrm{~h}}$ & $10^{\mathrm{h}}$ \\
\hline
\end{tabular}




\begin{tabular}{|c|c|c|c|c|}
\hline 30 & 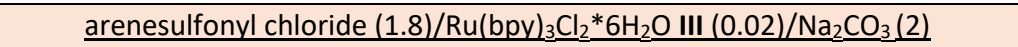 & Blue LED & $\underline{\mathrm{CH}_{3}} \underline{\mathrm{CN} / 4 h}$ & $42^{d}$ \\
\hline 31 & arenesulfonyl chloride (1.8)/Ru(bpy) ${ }_{3} \mathrm{Cl}_{2}{ }_{2} 6 \mathrm{H}_{2} \mathrm{O}$ III $(0.02) / \mathrm{Na}_{2} \mathrm{CO}_{3}(2)$ & Blue LED & $\mathrm{CH}_{3} \mathrm{CN} / 10 \mathrm{~h}$ & $57^{d}$ \\
\hline 32 & arenesulfonyl chloride $(1.8) / \mathrm{Ru}(\mathrm{bpm})_{3} \mathrm{Cl}_{2} * 6 \mathrm{H}_{2} \mathrm{O}$ IV $(0.02) / \mathrm{Na}_{2} \mathrm{CO}_{3}(2)$ & Blue LED & $\mathrm{CH}_{3} \mathrm{CN} / 4 \mathrm{~h}$ & 61 \\
\hline 33 & arenesulfonyl chloride $(1.8) / \mathrm{Ru}(\mathrm{bpz})_{3} \mathrm{Cl}_{2} * 6 \mathrm{H}_{2} \mathrm{O} \mathrm{V}(0.02) / \mathrm{Na}_{2} \mathrm{CO}_{3}$ (2) & Blue LED & $\mathrm{CH}_{3} \mathrm{CN} / 4 \mathrm{~h}$ & 57 \\
\hline 34 & arenesulfonyl chloride $(1.8) /\left[\mathrm{Cu}(\mathrm{dpp})_{2}\right] \mathrm{PF}_{6} \mathrm{VI}(0.02) / \mathrm{Na}_{2} \mathrm{CO}_{3}(2)$ & Green LED & $\mathrm{CH}_{3} \mathrm{CN} / 12 \mathrm{~h}$ & 0 \\
\hline 35 & arenesulfonyl chloride $(1.8) /\left[\mathrm{Cu}(\mathrm{dpp})_{2}\right] \mathrm{PF}_{6} \mathrm{VI}(0.02) / \mathrm{K}_{2} \mathrm{HPO}_{4}(2.5)$ & Green LED & DMSO/12h & 0 \\
\hline 36 & arenesulfonyl chloride $(1.8) /\left[\mathrm{Cu}(\text { dap })_{2}\right] \mathrm{PF}_{6} \mathrm{VII}(0.02) / \mathrm{K}_{2} \mathrm{HPO}_{4}(2.5)$ & Green LED & $\mathrm{CH}_{3} \mathrm{CN} / 12 \mathrm{~h}$ & 0 \\
\hline 37 & arenesulfonyl chloride $(1.8) /\left[\mathrm{Cu}(\text { dap })_{2}\right] \mathrm{PF}_{6} \mathrm{VII}(0.02) / \mathrm{Na}_{2} \mathrm{CO}_{3}(2)$ & Green LED & $\mathrm{CH}_{3} \mathrm{CN} / 12 \mathrm{~h}$ & 0 \\
\hline 38 & arenesulfonyl chloride $(2.2) / \mathrm{Ru}(\mathrm{bpy})_{3} \mathrm{Cl}_{2} * 6 \mathrm{H}_{2} \mathrm{O} \mathrm{III}(0.02) / \mathrm{Na}_{2} \mathrm{CO}_{3}(2) / \mathrm{TEMPO}(3)$ & Blue LED & $\mathrm{CH}_{3} \mathrm{CN} / 4 \mathrm{~h}$ & $0^{c}$ \\
\hline 39 & arenesulfonyl chloride $(2.2) / \mathrm{Ru}(\mathrm{bpy})_{3} \mathrm{Cl}_{2} * 6 \mathrm{H}_{2} \mathrm{O} \mathrm{III}(0.02) / \mathrm{Na}_{2} \mathrm{CO}_{3}(2)$ & NO & $\mathrm{CH}_{3} \mathrm{CN} / 4 \mathrm{~h}$ & $0^{c}$ \\
\hline 40 & arenesulfonyl chloride $(2.2) / \mathrm{Na}_{2} \mathrm{CO}_{3}(2)$ & Blue LED & $\mathrm{CH}_{3} \mathrm{CN} / 4 \mathrm{~h}$ & $0^{c}$ \\
\hline 41 & arenesulfonyl chloride (2.2)/Ru(bpy) ${ }_{3} \mathrm{Cl}_{2} * 6 \mathrm{H}_{2} \mathrm{O} \mathrm{III}(0.02) / \mathrm{Na}_{2} \mathrm{CO}_{3}(2) / \mathrm{TEMPO}$ (3) & Blue LED & $\mathrm{CH}_{3} \mathrm{CN} / 4 \mathrm{~h}$ & $0^{\mathrm{c}, \mathrm{f}}$ \\
\hline 42 & arenesulfonyl chloride (2.2)/Ru(bpy) ${ }_{3} \mathrm{Cl}_{2} * 6 \mathrm{H}_{2} \mathrm{O} \mathrm{III}(0.02) / \mathrm{Na}_{2} \mathrm{CO}_{3}(2)$ & NO & $\mathrm{CH}_{3} \mathrm{CN} / 4 \mathrm{~h}$ & $0^{\mathrm{c}, \mathrm{f}}$ \\
\hline 43 & arenesulfonyl chloride $(2.2) / \mathrm{Na}_{2} \mathrm{CO}_{3}(2)$ & Blue LED & $\mathrm{CH}_{3} \mathrm{CN} / 4 \mathrm{~h}$ & $0^{\mathrm{c}, \mathrm{f}}$ \\
\hline 44 & arenesulfonyl chloride (2.2)/Ru(bpy) ${ }_{3} \mathrm{Cl}_{2} * 6 \mathrm{H}_{2} \mathrm{O} \mathrm{III}(0.02) / \mathrm{Na}_{2} \mathrm{CO}_{3}(2) / \mathrm{TEMPO}$ (3) & Blue LED & $\mathrm{CH}_{3} \mathrm{CN} / 4 \mathrm{~h}$ & $0^{c, d}$ \\
\hline 45 & arenesulfonyl chloride (2.2)/Ru(bpy) ${ }_{3} \mathrm{Cl}_{2} * 6 \mathrm{H}_{2} \mathrm{O} \mathrm{III}(0.02) / \mathrm{Na}_{2} \mathrm{CO}_{3}(2)$ & NO & $\mathrm{CH}_{3} \mathrm{CN} / 4 \mathrm{~h}$ & $0^{c, d}$ \\
\hline 46 & arenesulfonyl chloride $(2.2) / \mathrm{Na}_{2} \mathrm{CO}_{3}(2)$ & Blue LED & $\mathrm{CH}_{3} \mathrm{CN} / 4 \mathrm{~h}$ & $0^{c, d}$ \\
\hline
\end{tabular}

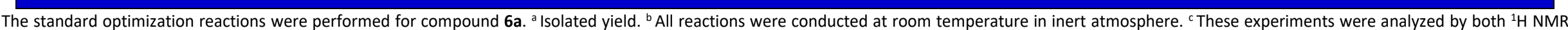

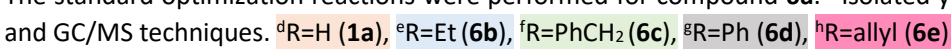


(B) Copies ${ }^{1} \mathrm{H}$ and ${ }^{13} \mathrm{C}$ NMR spectra. 


\section{Compound 5a (NMR in $\left.\mathrm{CDCl}_{3}\right)$}

SpinWorks 4: ISM 32-1
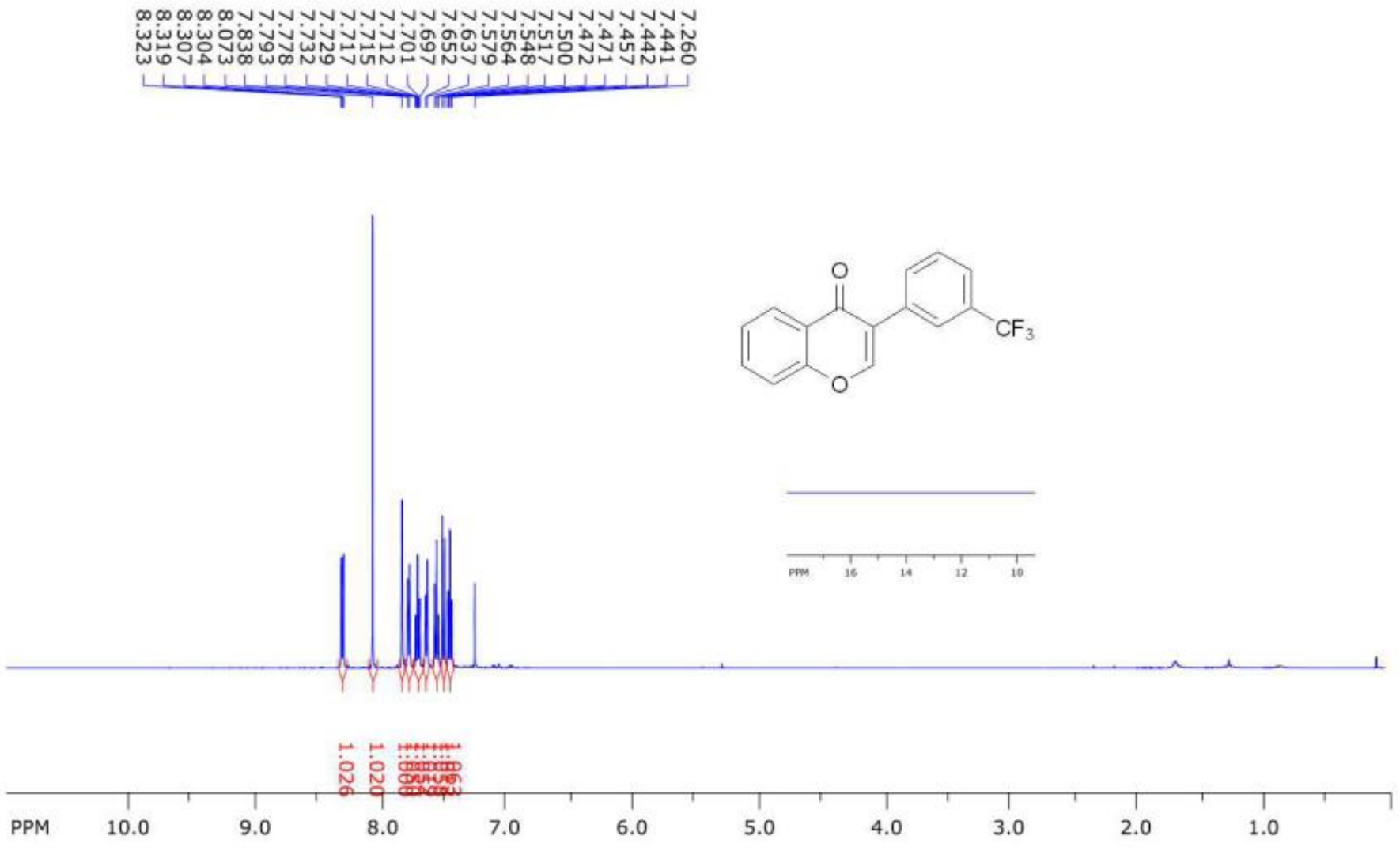

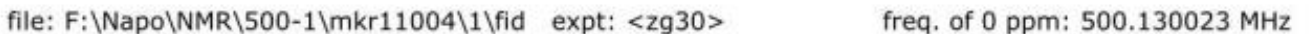

transmitter freq.: $500.133001 \mathrm{MHz}$

time domain size: 65536 points

width: $12335.53 \mathrm{~Hz}=24.6645 \mathrm{ppm}=0.188225 \mathrm{~Hz} / \mathrm{pt} \quad$ Hz/cm: $219.727 \mathrm{ppm} / \mathrm{cm}: 0.43934$

number of scans: 24 


\section{Compound 5a (NMR in $\left.\mathrm{CDCl}_{3}\right)$}

SpinWorks 4: ISM 32-1
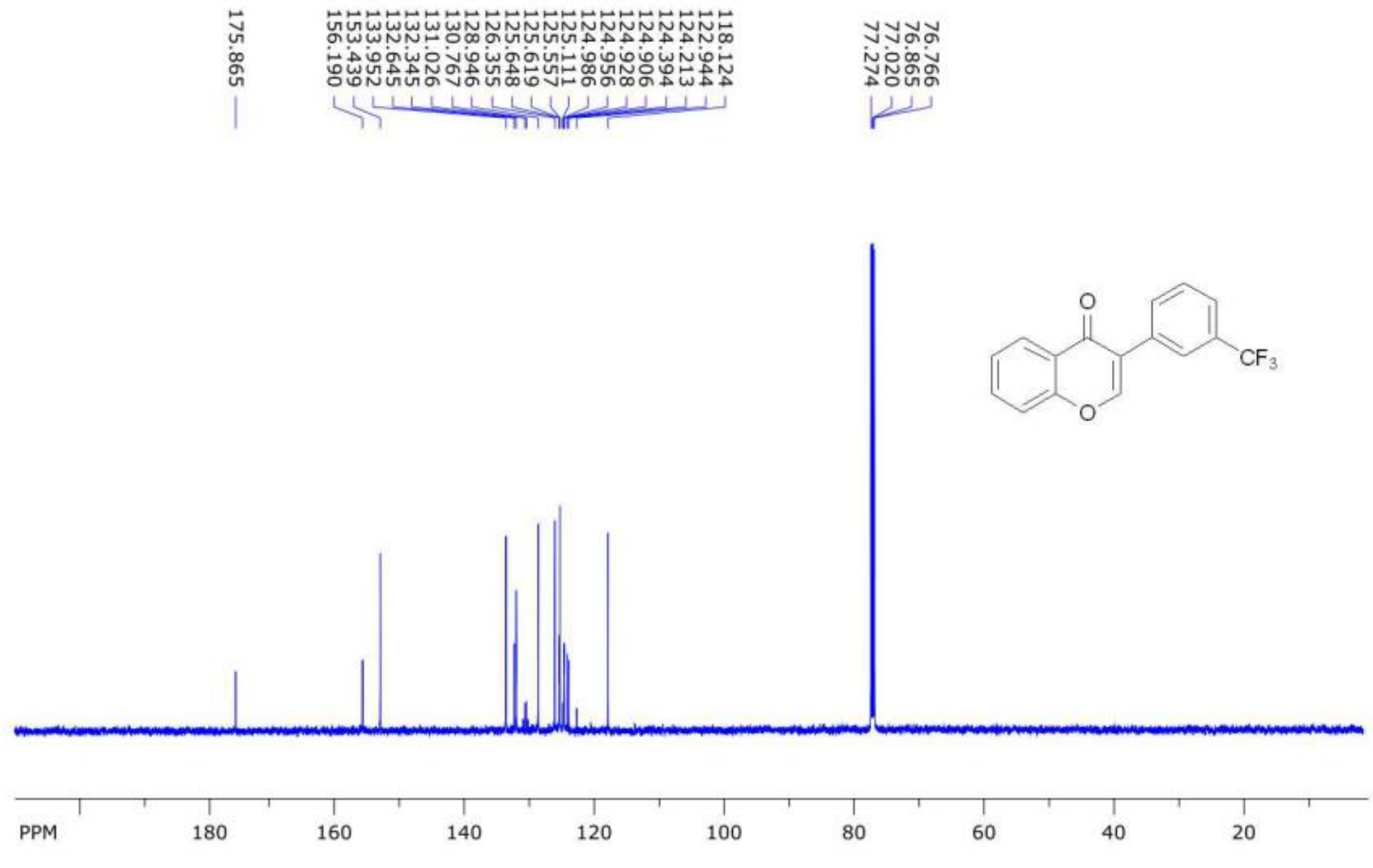

file: $F: \backslash N a p o \backslash N M R \backslash 500-1 \backslash m k r 11004 \backslash 2 \backslash f i d$ expt: <zgpg30> transmitter freq.: $125.772879 \mathrm{MHz}$

time domain size: 65536 points

width: $36057.69 \mathrm{~Hz}=286.6889 \mathrm{ppm}=0.550197 \mathrm{~Hz} / \mathrm{pt}$

number of scans: 512

freq. of 0 ppm: $125.757794 \mathrm{MHz}$ processed size: 32768 complex points

LB: 2.000 GF: 0.0000

$\mathrm{Hz} / \mathrm{cm}: 1052.756 \mathrm{ppm} / \mathrm{cm}: 8.37029$ 


\section{Compound 5b (NMR in $\mathrm{CDCl}_{3}$ )}

SpinWorks 4: ISM 207 1H CDCl3
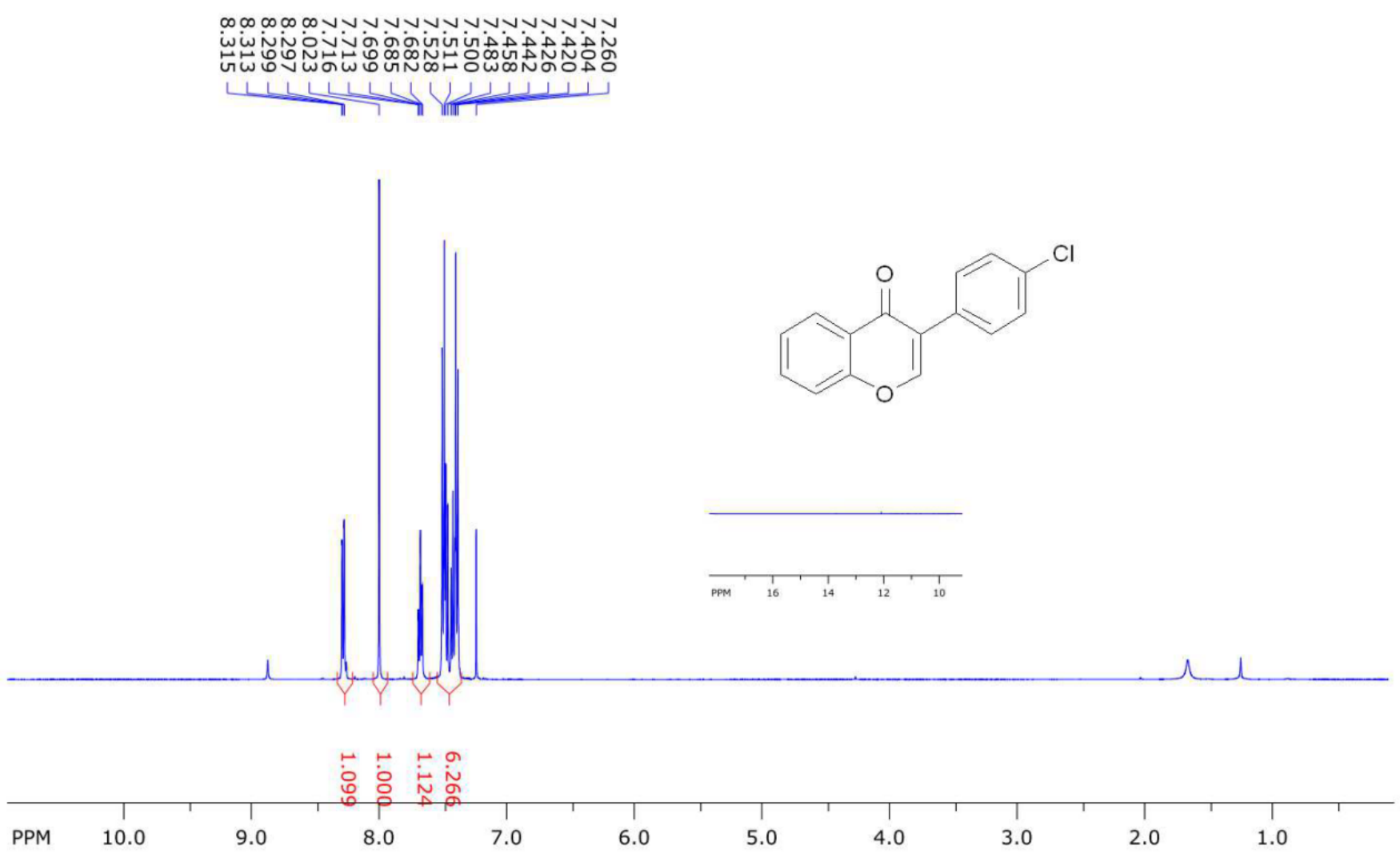

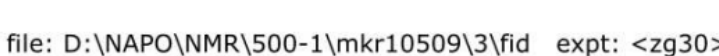
transmitter freq.: $500.133001 \mathrm{MHz}$

time domain size: 65536 points

freq. of $0 \mathrm{ppm}$ : $500.130023 \mathrm{MHz}$

width: $12335.53 \mathrm{~Hz}=24.6645 \mathrm{ppm}=0.188225 \mathrm{~Hz} / \mathrm{pt}$

processed size: 65536 complex points

LB: 0.300 GF: 0.0000

number of scans: 32

$\mathrm{Hz} / \mathrm{cm}: 217.236 \mathrm{ppm} / \mathrm{cm}: 0.43436$ 


\section{Compound 5b (NMR in $\left.\mathrm{CDCl}_{3}\right)$}

SpinWorks 4: ISM $20713 \mathrm{C} \mathrm{CDCl} 3$

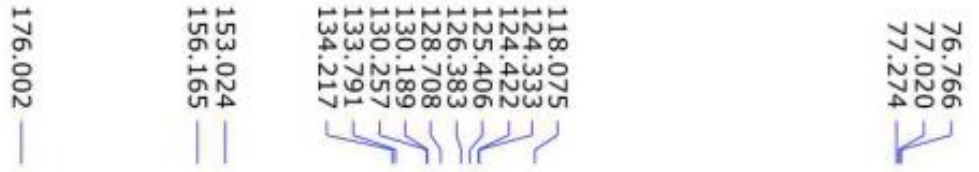
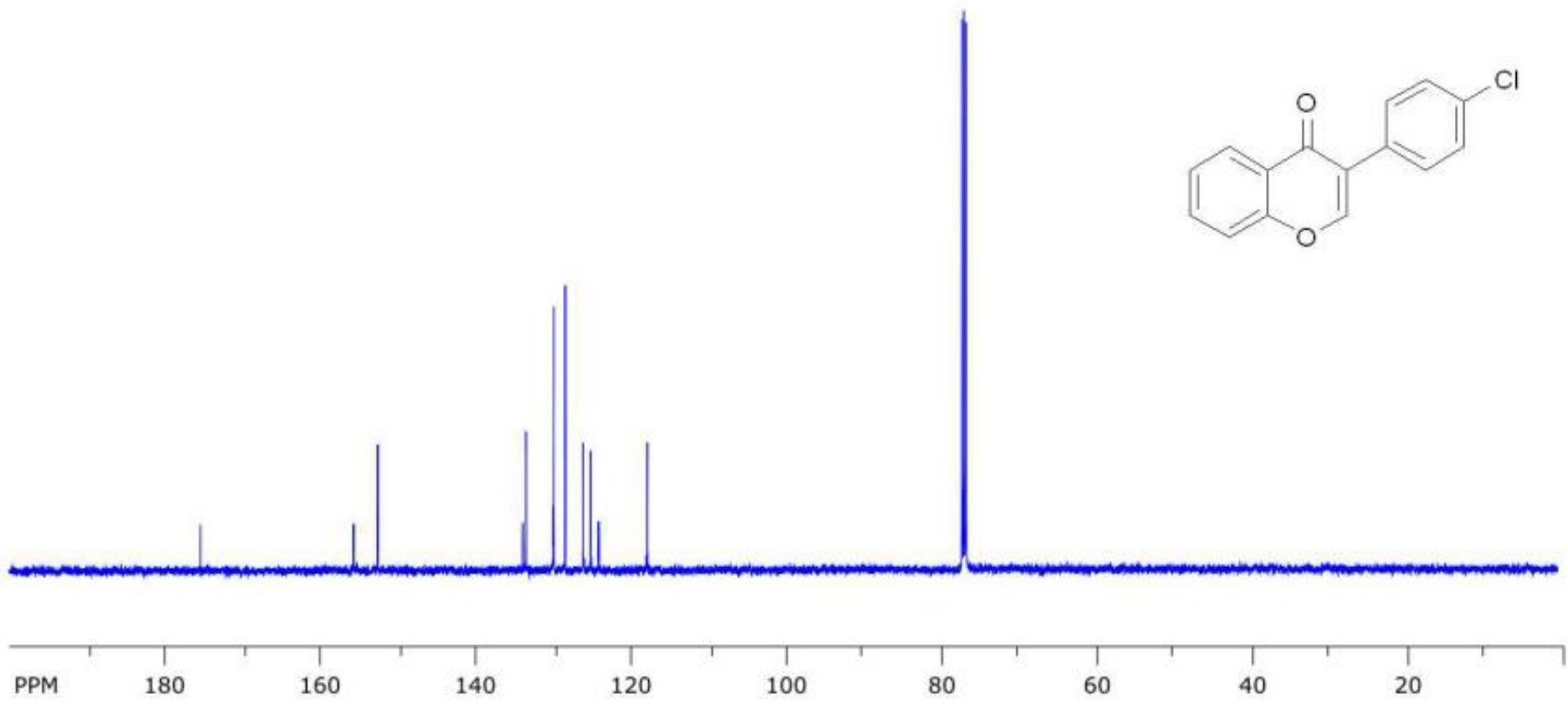

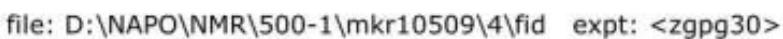
transmitter freq.: $125.772879 \mathrm{MHz}$

time domain size: 65536 points

width: $36057.69 \mathrm{~Hz}=286.6889 \mathrm{ppm}=0.550197 \mathrm{~Hz} / \mathrm{pt}$

number of scans: 512

freq. of $0 \mathrm{ppm}: 125.757794 \mathrm{MHz}$

processed size: 32768 complex points

LB: 2.000 GF: 0.0000

$\mathrm{Hz} / \mathrm{cm}: 1009.934 \mathrm{ppm} / \mathrm{cm}: 8.02983$ 


\section{Compound 5c (NMR in $\left.\mathrm{CDCl}_{3}\right)$}

SpinWorks 4: IVA $2871 \mathrm{H}$
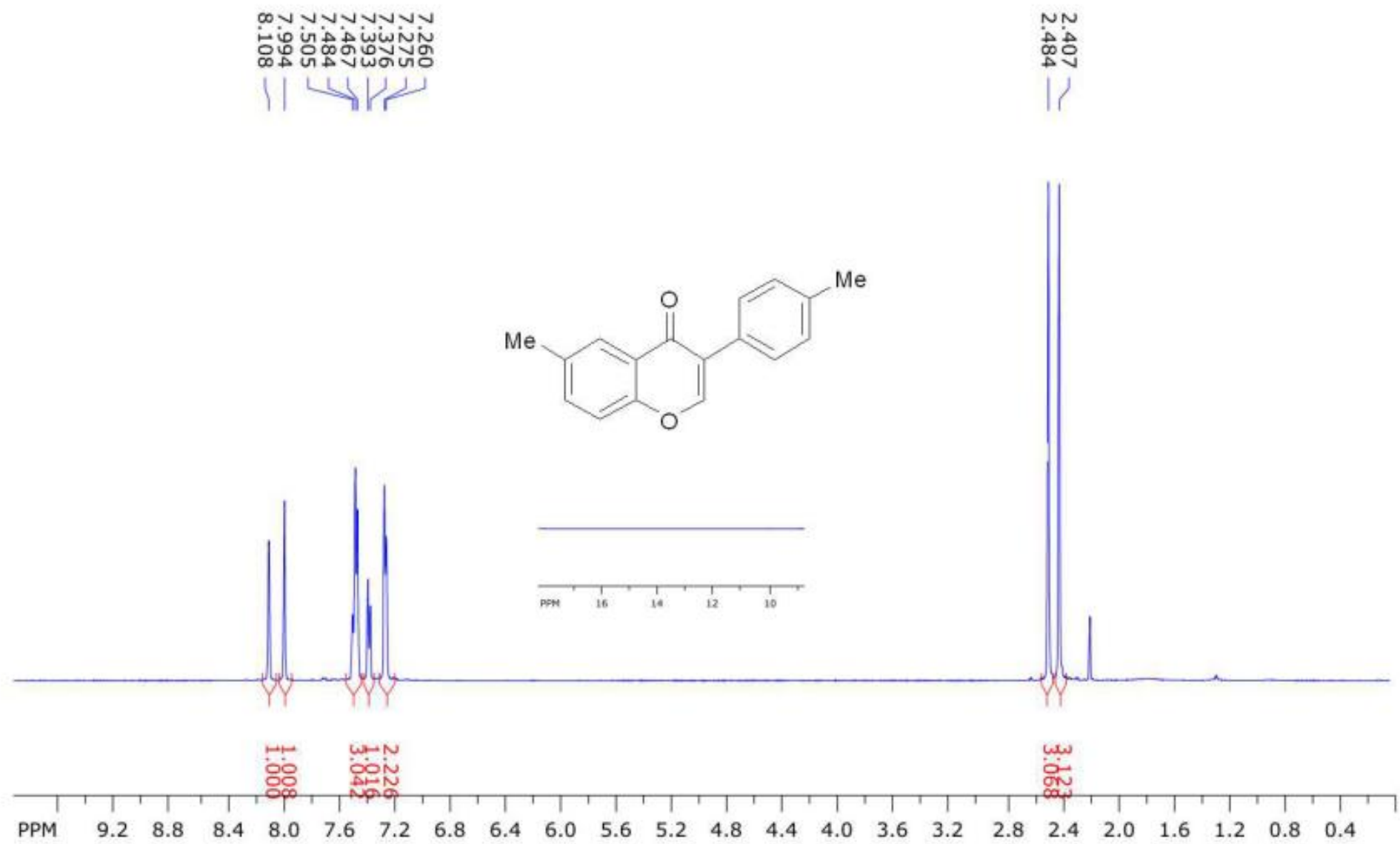

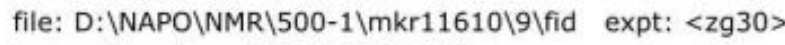
transmitter freq.: $500.133001 \mathrm{MHz}$

time domain size: 65536 points

freq. of $0 \mathrm{ppm}$ : $500.130016 \mathrm{MHz}$

processed size: 65536 complex points

width: $12335.53 \mathrm{~Hz}=24.6645 \mathrm{ppm}=0.188225 \mathrm{~Hz} / \mathrm{pt}$

LB: 0.300 GF: 0.0000

number of scans: 32

$\mathrm{Hz} / \mathrm{cm}: 198.678 \mathrm{ppm} / \mathrm{cm}: 0.39725$ 


\section{Compound 5c (NMR in $\left.\mathrm{CDCl}_{3}\right)$}

SpinWorks 4: ISM 287 13C
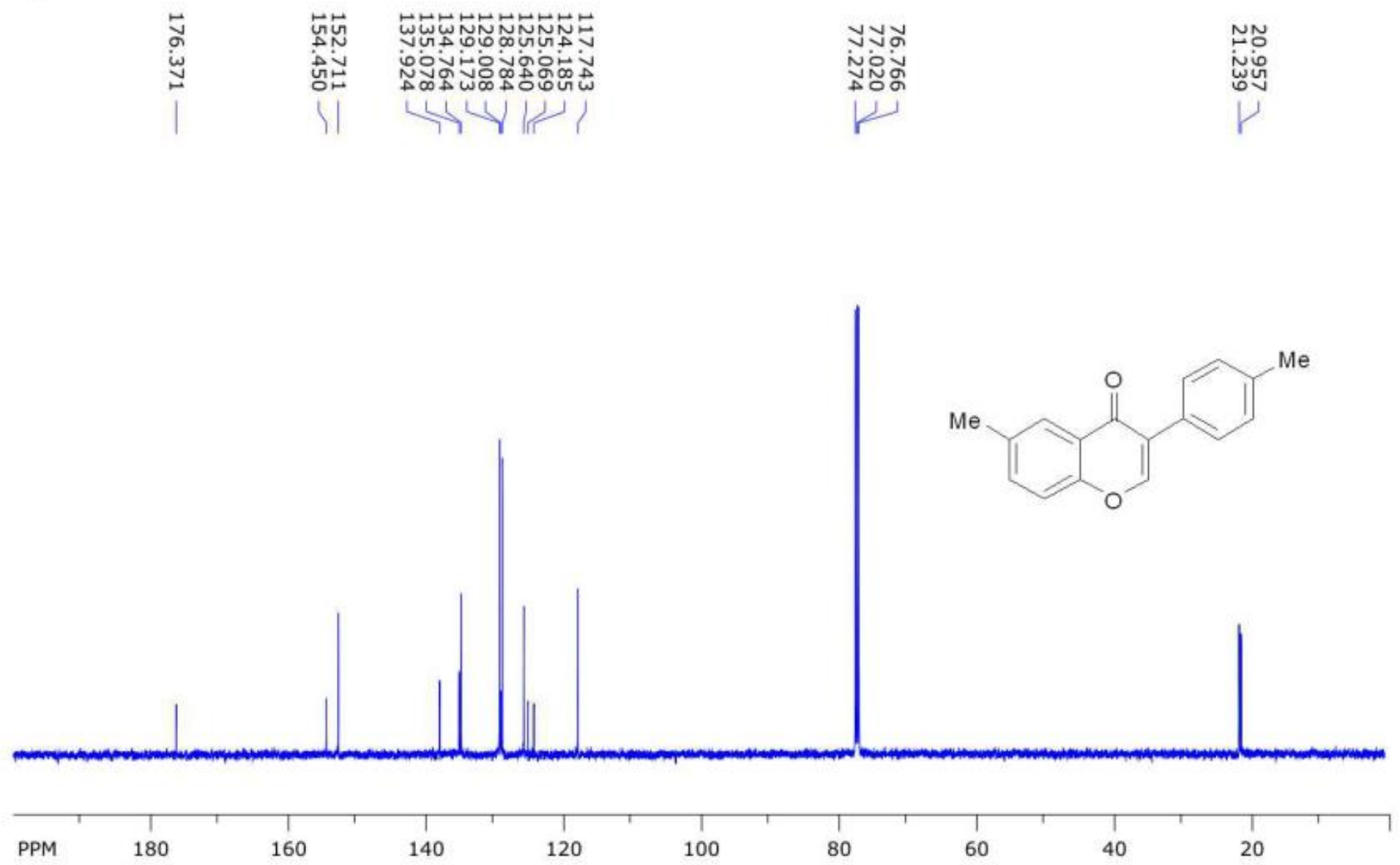

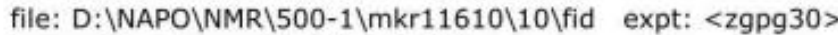
transmitter freq.: $125.772879 \mathrm{MHz}$

time domain size: 65536 points

freq. of $0 \mathrm{ppm}: 125.757797 \mathrm{MHz}$

processed size: 32768 complex points

width: $36057.69 \mathrm{~Hz}=286.6889 \mathrm{ppm}=0.550197 \mathrm{~Hz} / \mathrm{pt}$

LB: 2.000 GF: 0.0000

number of scans: 380

$\mathrm{Hz} / \mathrm{cm}: 1006.744 \mathrm{ppm} / \mathrm{cm}: 8.00446$ 


\section{Compound 5d (NMR in $\mathrm{CDCl}_{3}$ )}

SpinWorks 4: ISM 257 1H
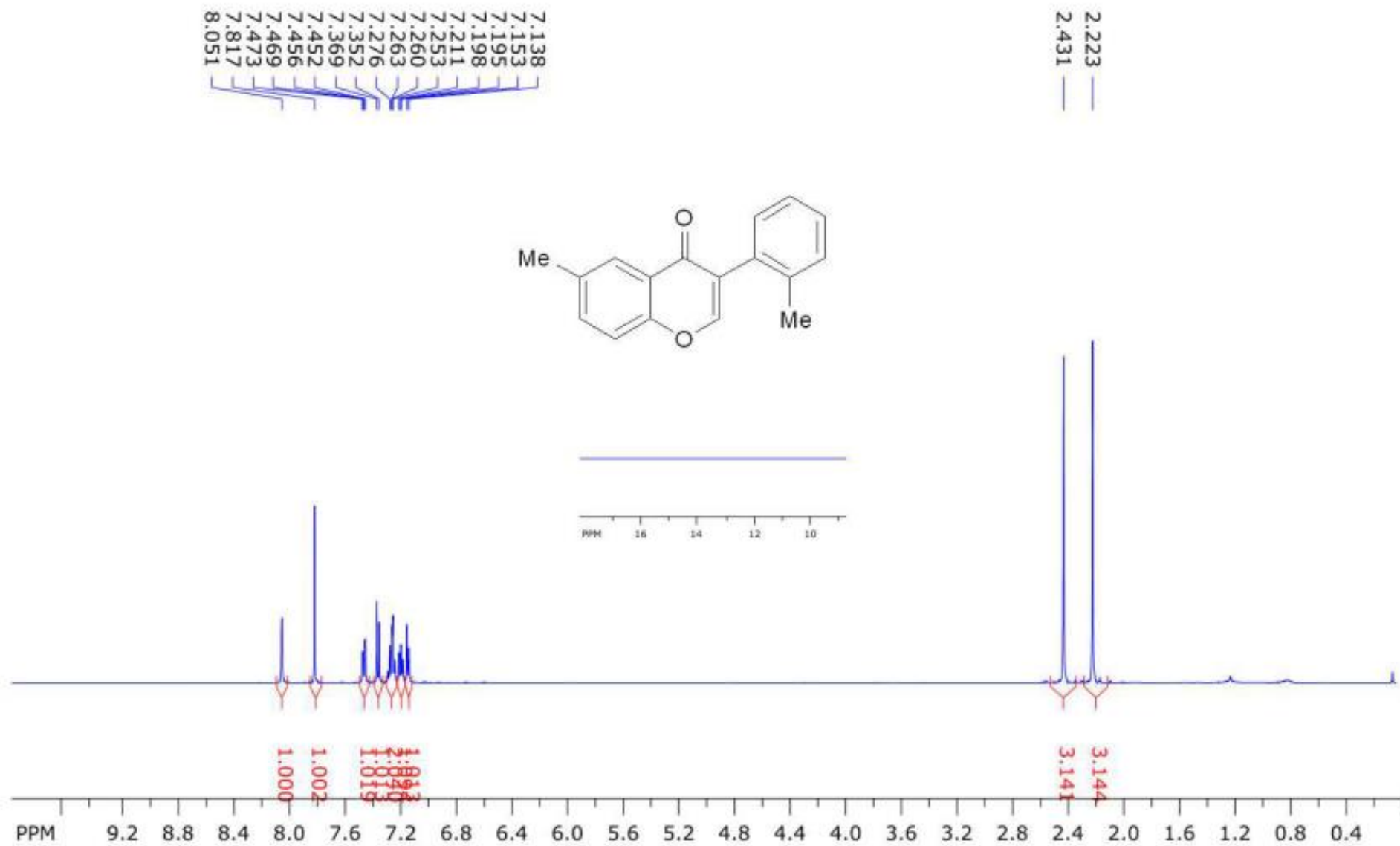

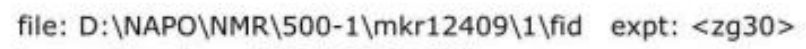
transmitter freq.: $500.133001 \mathrm{MHz}$

time domain size: 65536 points

width: $12335.53 \mathrm{~Hz}=24.6645 \mathrm{ppm}=0.188225 \mathrm{~Hz} / \mathrm{pt}$

number of scans: 32 freq. of 0 ppm: $500.130048 \mathrm{MHz}$

processed size: 65536 complex points

LB: 0.300 GF: 0.0000

$\mathrm{Hz} / \mathrm{cm}: 199.224 \mathrm{ppm} / \mathrm{cm}: 0.39834$ 


\section{Compound 5d (NMR in $\left.\mathrm{CDCl}_{3}\right)$}

SpinWorks 4: ISM 257 13C
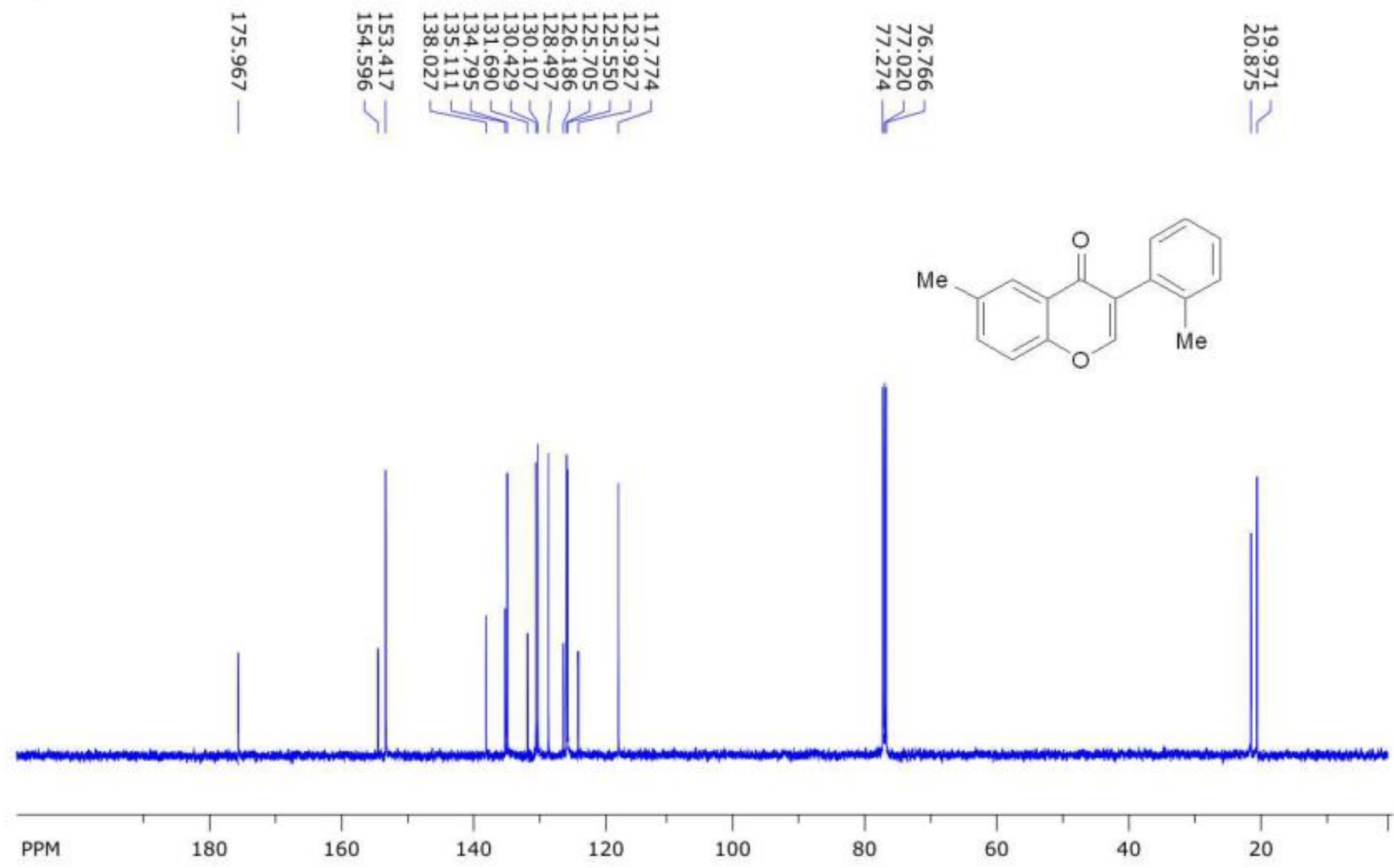

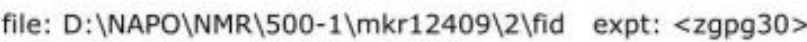
transmitter freq.: $125.772879 \mathrm{MHz}$

freq. of $0 \mathrm{ppm}: 125.757806 \mathrm{MHz}$

time domain size: 65536 points

processed size: 32768 complex points

width: $36057.69 \mathrm{~Hz}=286.6889 \mathrm{ppm}=0.550197 \mathrm{~Hz} / \mathrm{pt}$

LB: 2.000 GF: 0.0000

number of scans: 148 


\section{Compound 5e (NMR in $\left.\mathrm{CDCl}_{3}\right)$}

SpinWorks 4: ISM 15-2 $1 \mathrm{H}$

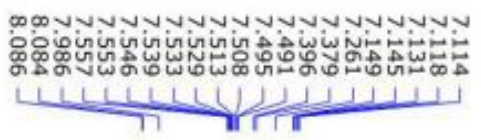

$\frac{\mathrm{LL} L \mathrm{~L}}{7}$

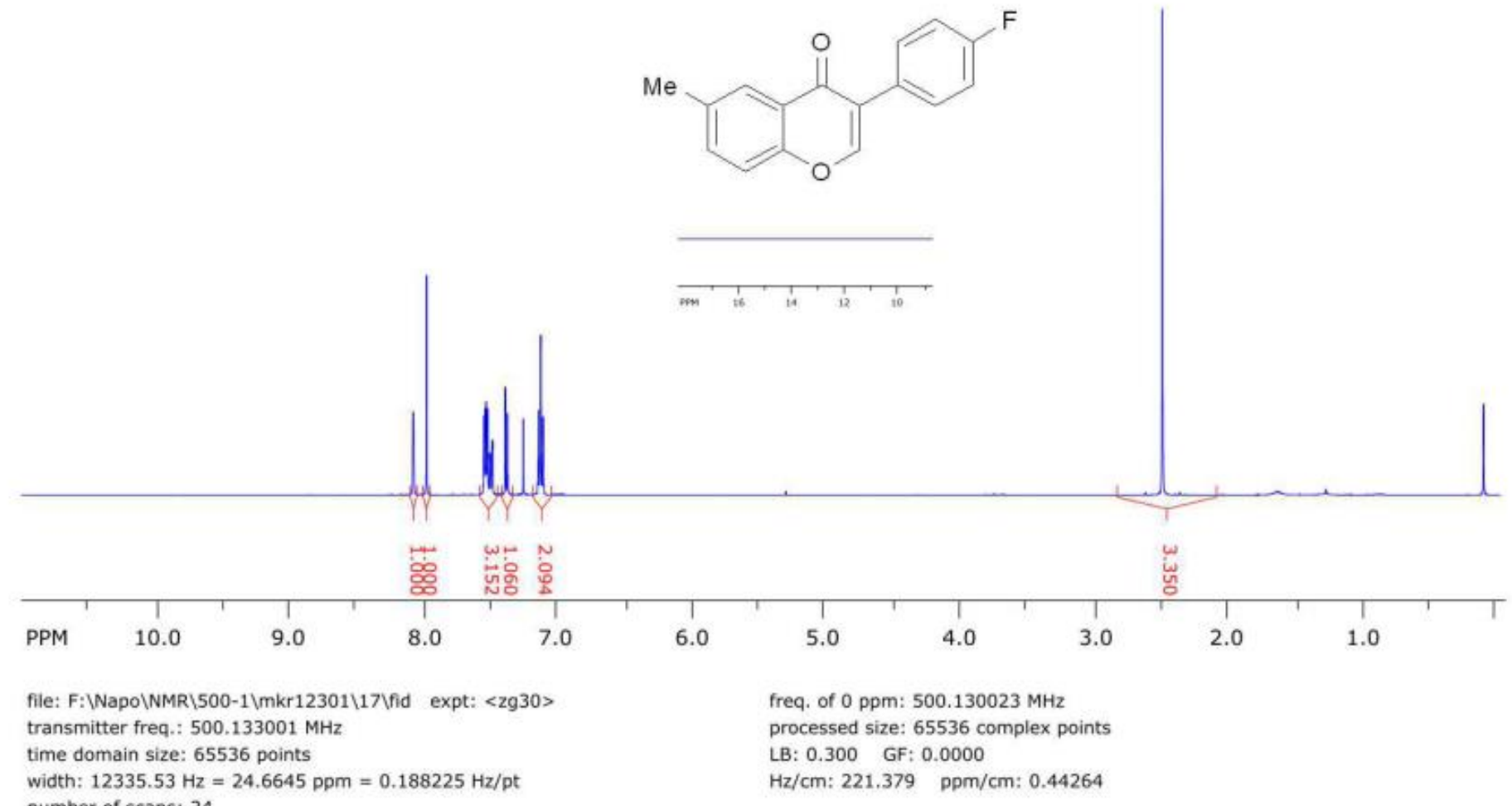




\section{Compound 5e (NMR in $\left.\mathrm{CDCl}_{3}\right)$}

SpinWorks 4: ISM 15-2 13C

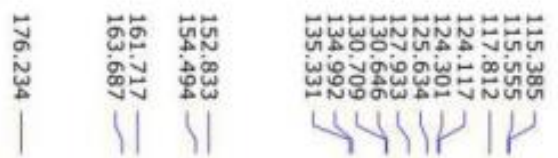<smiles>Cc1ccc2occ(-c3ccc(F)cc3)c(=O)c2c1</smiles>

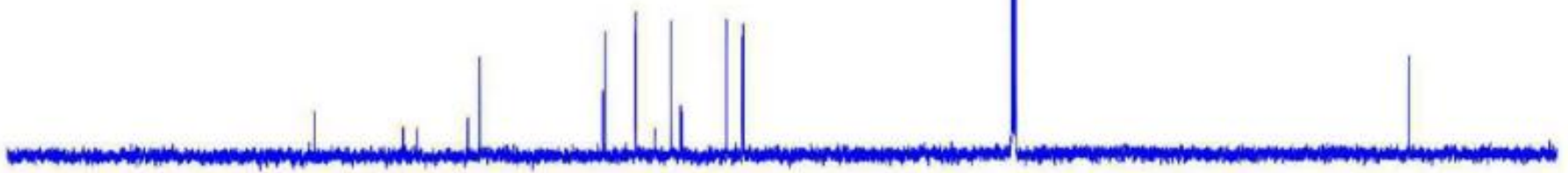

PPM

200

180

160

140

120

100

80

60

40

20

file: F: WapolWMR $500-1 \backslash m k r 12301 \backslash 18 \backslash$ fid expt: <zgpg30> transmitter freq.: $125.772879 \mathrm{MHz}$

freq. of $0 \mathrm{ppm}: 125.757793 \mathrm{MHz}$

time domain size: 65536 points

processed size: 32768 complex points

LB: 2.000 GF: 0.0000

width: $36057.69 \mathrm{~Hz}=286.6889 \mathrm{ppm}=0.550197 \mathrm{~Hz} / \mathrm{pt}$

$\mathrm{Hz} / \mathrm{cm}: 1105.547 \mathrm{ppm} / \mathrm{cm}: 8.79003$

number of scans: 256 


\section{Compound $5 f$ (NMR in $\left.\mathrm{CDCl}_{3}\right)$}

SpinWorks 4: ISM 13-1 $1 \mathrm{H}$
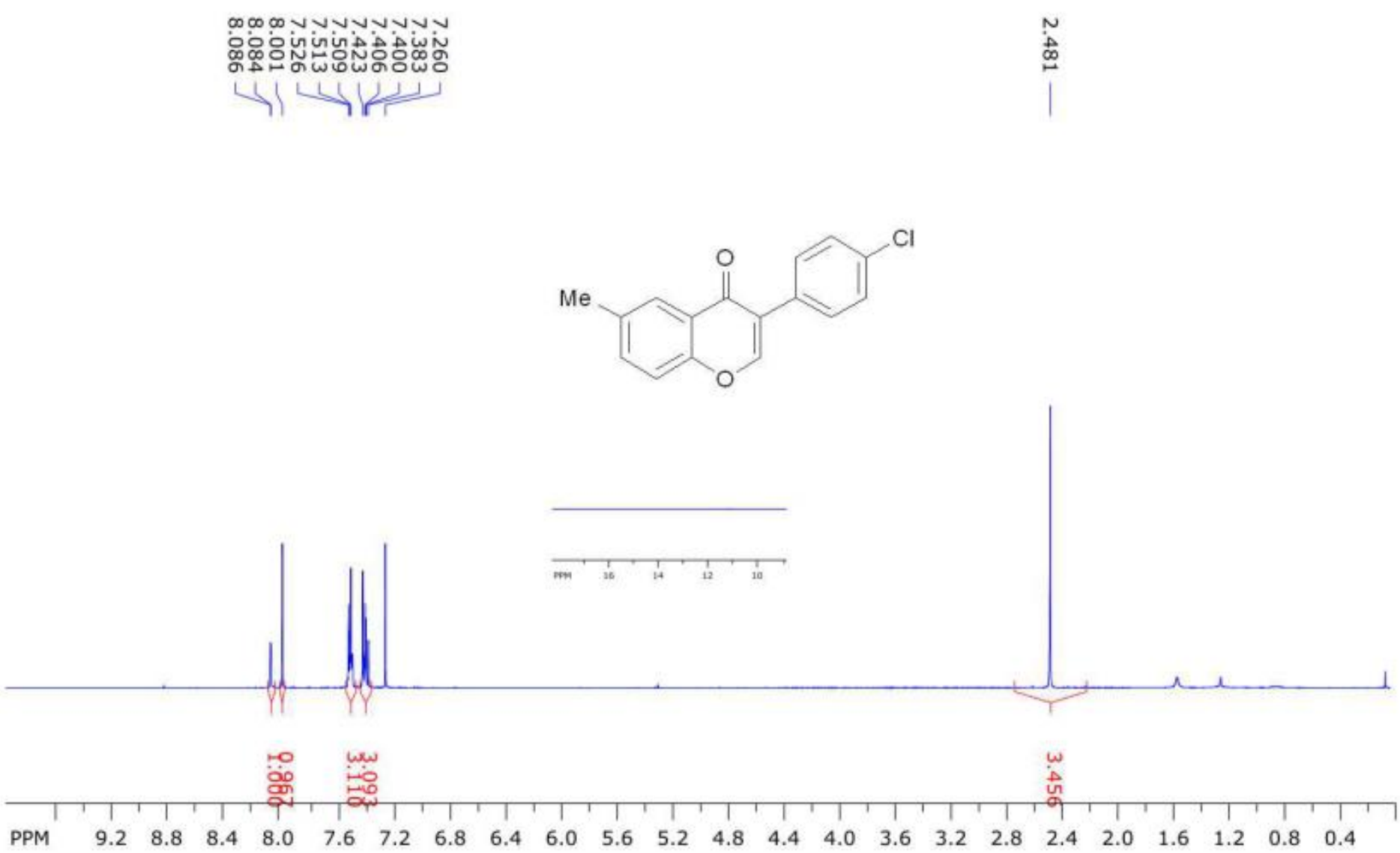

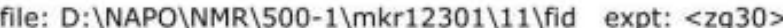
transmitter freq.: $500.133001 \mathrm{MHz}$

time domain size: 65536 points

width: $12335.53 \mathrm{~Hz}=24.6645 \mathrm{ppm}=0.188225 \mathrm{~Hz} / \mathrm{pt}$

number of scans: 24 freq. of 0 ppm: $500.130023 \mathrm{MHz}$

processed size: 65536 complex points

LB: 0.300 GF: 0.0000

$\mathrm{Hz} / \mathrm{cm}: 199.224 \mathrm{ppm} / \mathrm{cm}: 0.39834$ 


\section{Compound 5g (NMR in $\mathrm{CDCl}_{3}$ )}

SpinWorks 4: ISM 77 1H
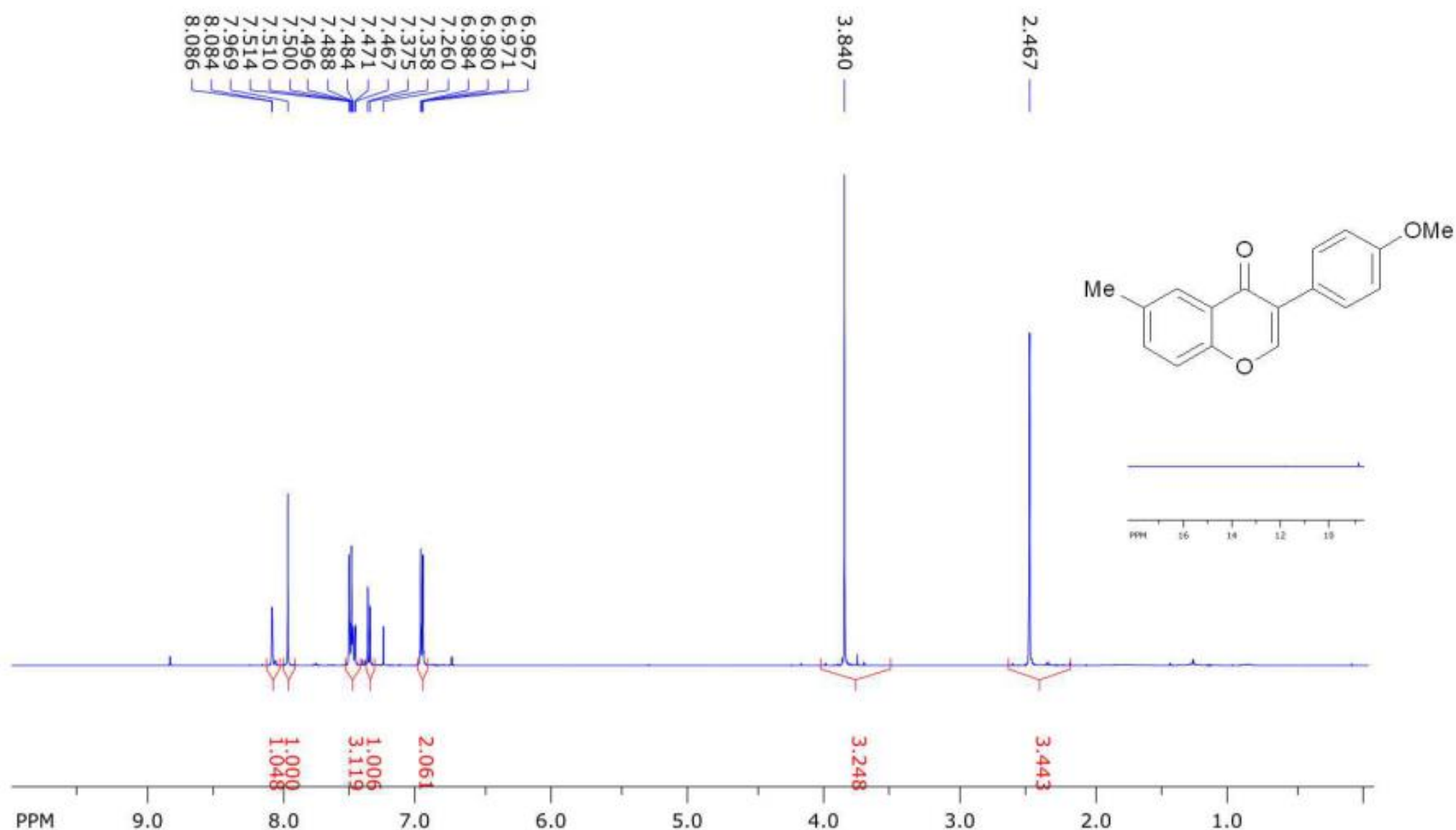

file: D: \NAPO\NMR $500-1 \backslash m k r 12706 \backslash 21 \backslash$ fid expt: $<z g 30>$ transmitter freq.: $500.133001 \mathrm{MHz}$

time domain size: 65536 points

freq. of $0 \mathrm{ppm}$ : $500.130023 \mathrm{MHz}$

width: $12335.53 \mathrm{~Hz}=24.6645 \mathrm{ppm}=0.188225 \mathrm{~Hz} / \mathrm{pt}$ processed size: 65536 complex points width: $12335.53 \mathrm{~Hz}$ 


\section{Compound 5g (NMR in $\left.\mathrm{CDCl}_{3}\right)$}

SpinWorks 4: ISM $7713 C$
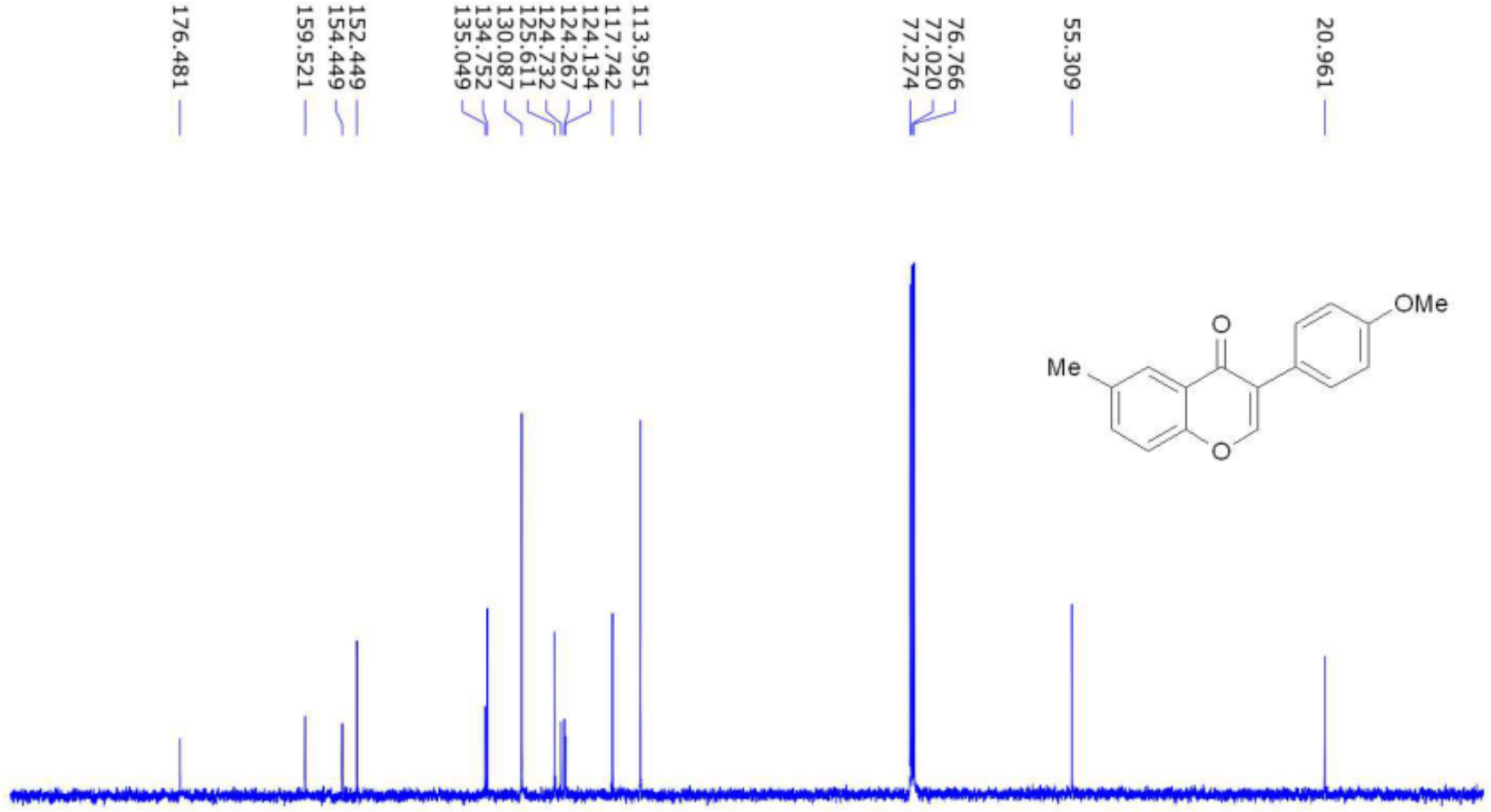

PPM

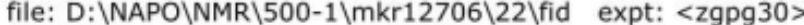
transmitter freq.: $125.772879 \mathrm{MHz}$

time domain size: 65536 points

width: $36057.69 \mathrm{~Hz}=286.6889 \mathrm{ppm}=0.550197 \mathrm{~Hz} / \mathrm{pt}$ number of scans: 256 freq. of 0 ppm: $125.757796 \mathrm{MHz}$ processed size: 32768 complex points LB: 2.000 GF: 0.0000

$\mathrm{Hz} / \mathrm{cm}: 1006.744$ ppm/cm: 8.00446 


\section{Compound 5h (NMR in $\left.\mathrm{CDCl}_{3}\right)$}

SpinWorks 4: ISM $1261 \mathrm{H}$
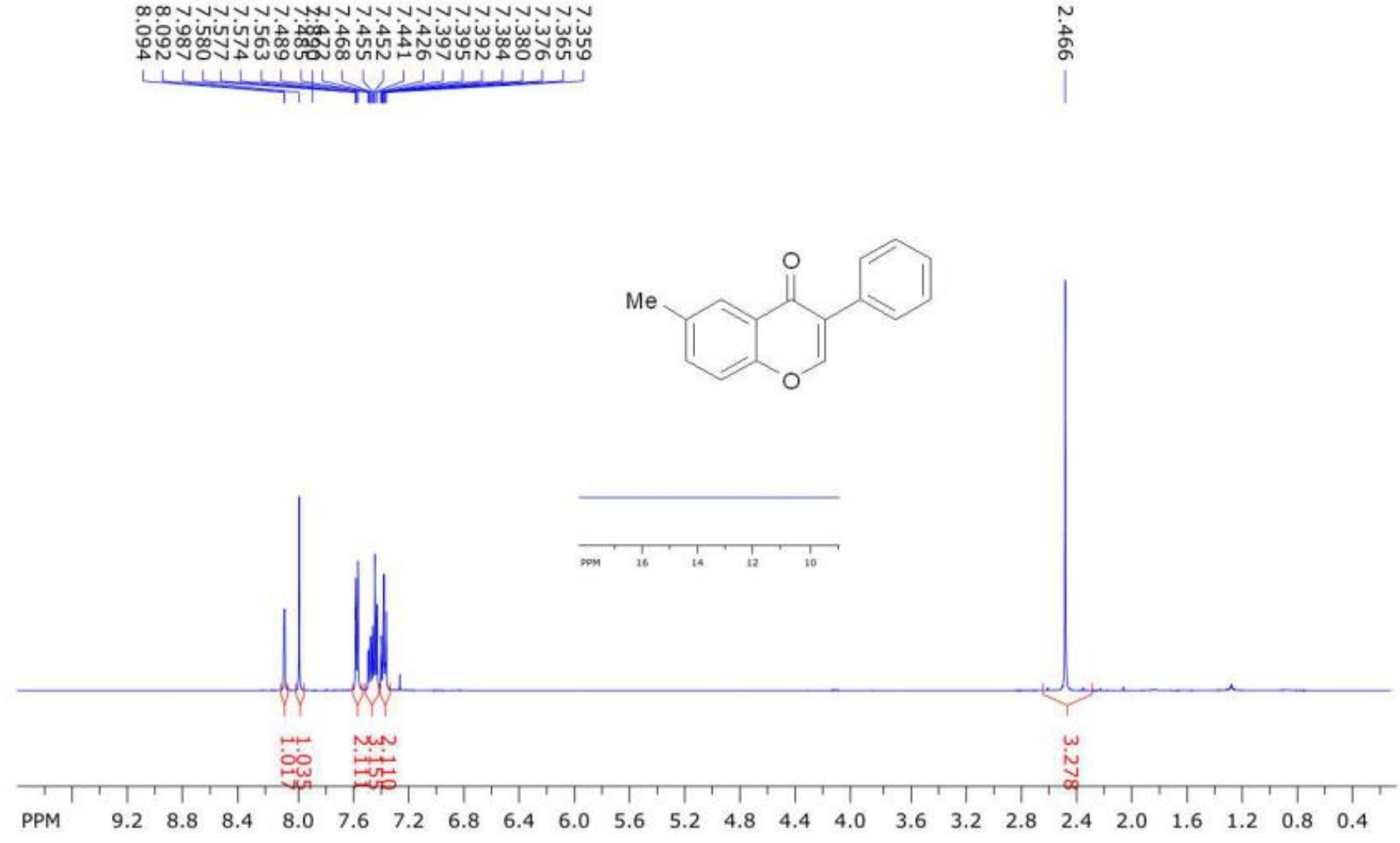

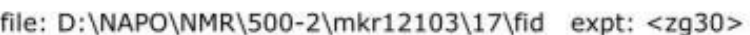
transmitter freq.: $500.133001 \mathrm{MHz}$

time domain size: 65536 points

freq. of 0 ppm: $500.130022 \mathrm{MHz}$

width: $12335.53 \mathrm{~Hz}=24.6645 \mathrm{ppm}=0.188225 \mathrm{~Hz} / \mathrm{pt}$

processed size: 65536 complex points

number of scans: 24 


\section{Compound 5h (NMR in $\left.\mathrm{CDCl}_{3}\right)$}

SpinWorks 4: ISM $12613 \mathrm{C}$

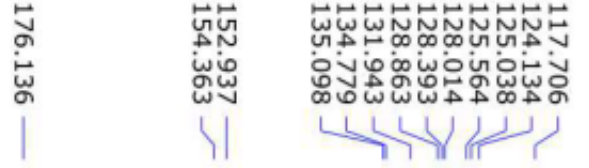

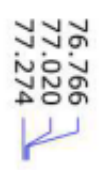

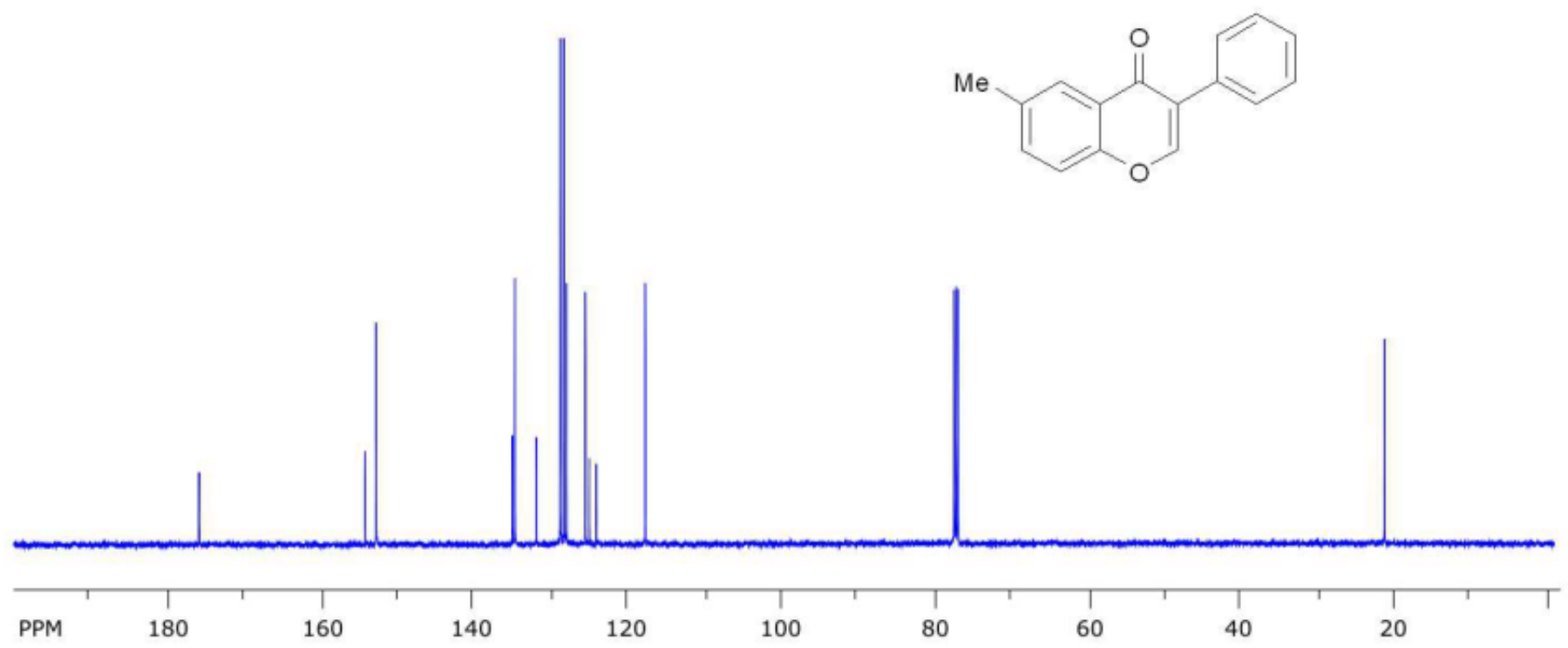

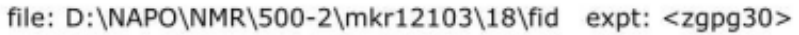
transmitter freq.: $125.772879 \mathrm{MHz}$

time domain size: 65536 points

freq. of $0 \mathrm{ppm}: 125.757804 \mathrm{MHz}$

processed size: 32768 complex points

width: $36057.69 \mathrm{~Hz}=286.6889 \mathrm{ppm}=0.550197 \mathrm{~Hz} / \mathrm{pt}$

number of scans: 512

LB: 2.000 GF: 0.0000

$\mathrm{Hz} / \mathrm{cm}: 1014.721 \mathrm{ppm} / \mathrm{cm}: 8.06788$ 


\section{Compound 5i (NMR in $\mathrm{CDCl}_{3}$ )}

SpinWorks 4: ISM $3131 \mathrm{H} \mathrm{CDCl3}$
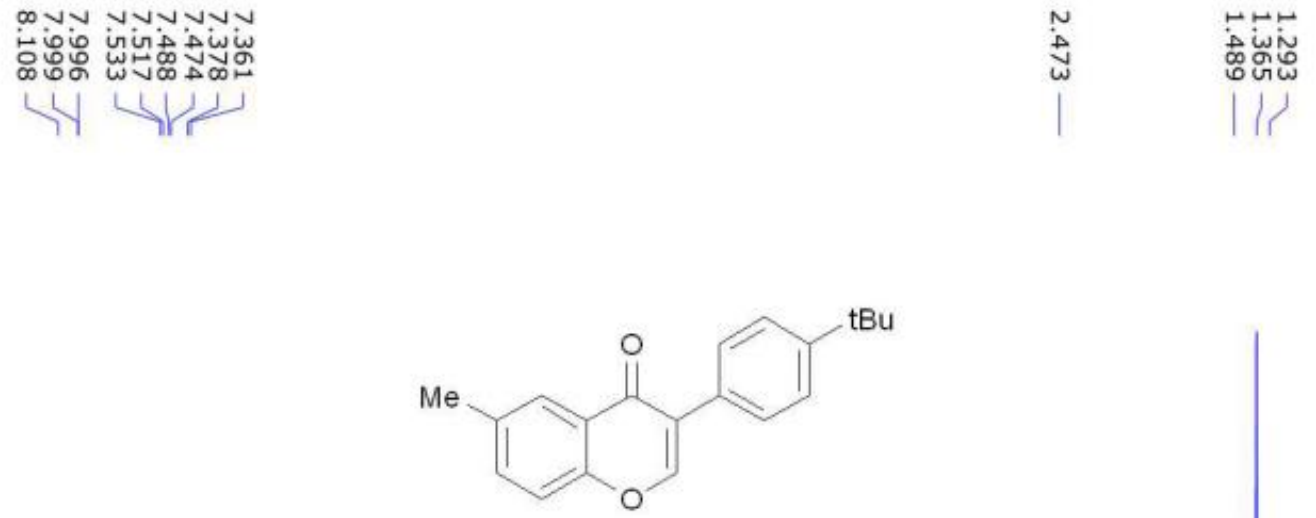
Bu
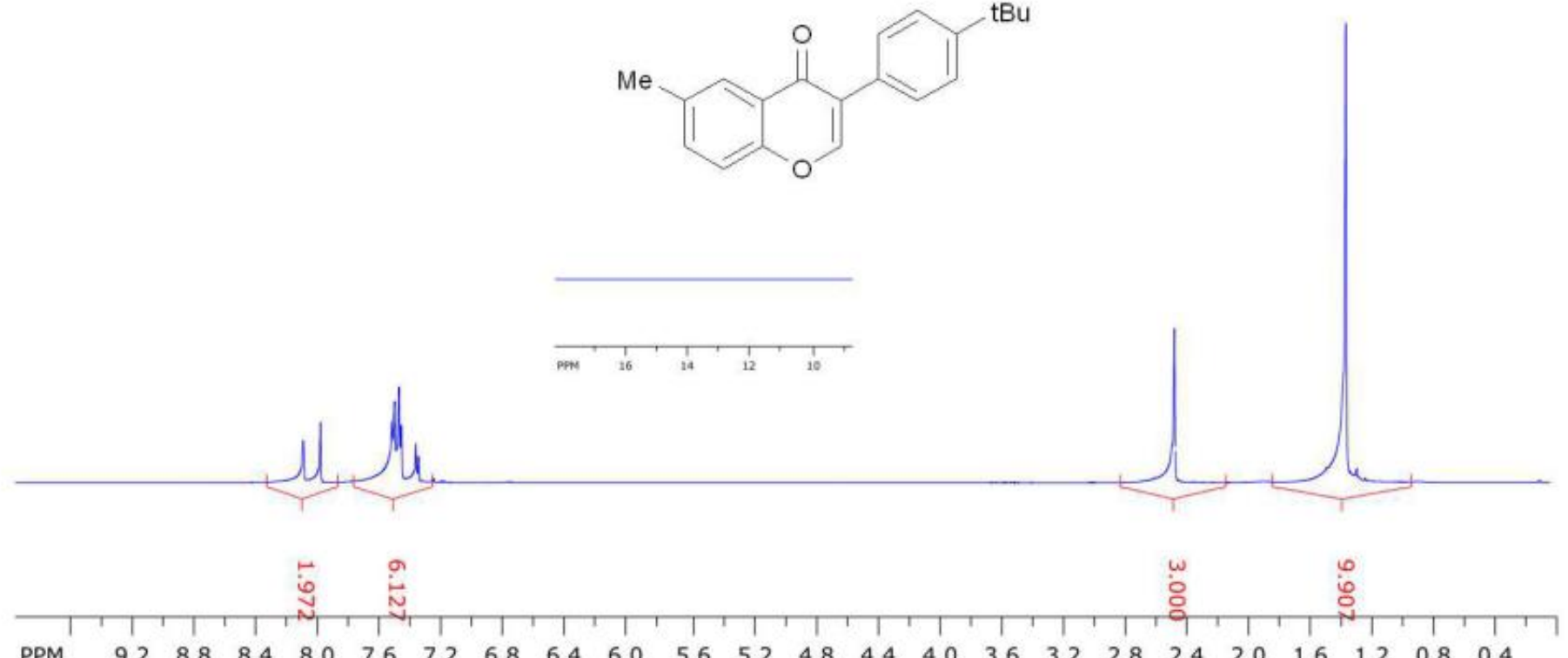

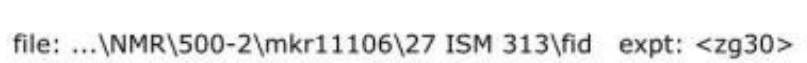
transmitter freq.: $500.133001 \mathrm{MHz}$

time domain size: 65536 points

freq. of 0 ppm: $500.130020 \mathrm{MHz}$

processed size: 65536 complex points

LB: 0.300 GF: 0.0000

width: $12335.53 \mathrm{~Hz}=24.6645 \mathrm{ppm}=0.188225 \mathrm{~Hz} / \mathrm{pt}$

$\mathrm{Hz} / \mathrm{cm}: 198.678 \mathrm{ppm} / \mathrm{cm}: 0.39725$

number of scans: 24 


\section{Compound 5i (NMR in $\mathrm{CDCl}_{3}$ )}

SpinWorks 4: ISM 313 13C CDCI3
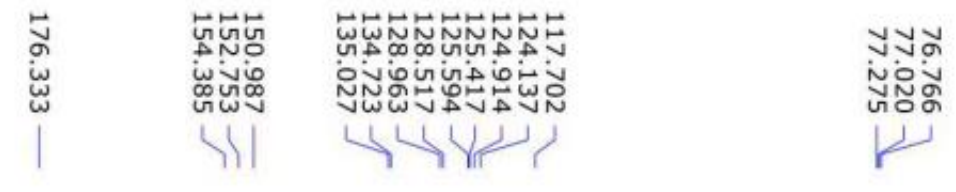

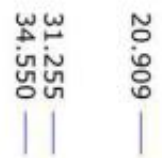

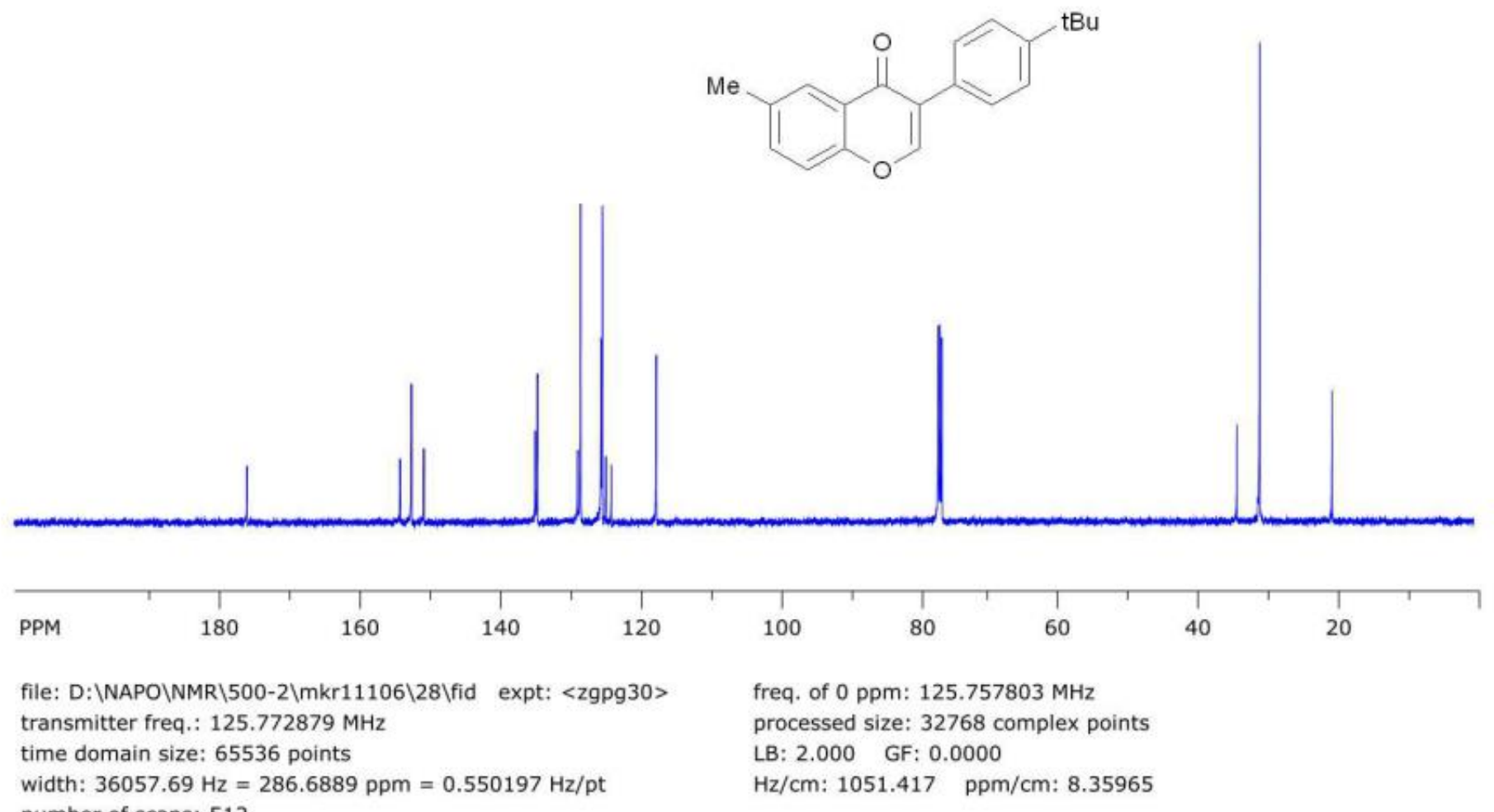

number of scans: 512 


\section{Compound 5j (NMR in $\mathrm{CDCl}_{3}$ )}

SpinWorks 4: ISM 269 1H

$$
\infty \pi v N n v N v a g
$$

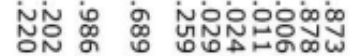

$\checkmark 11$ Yyt
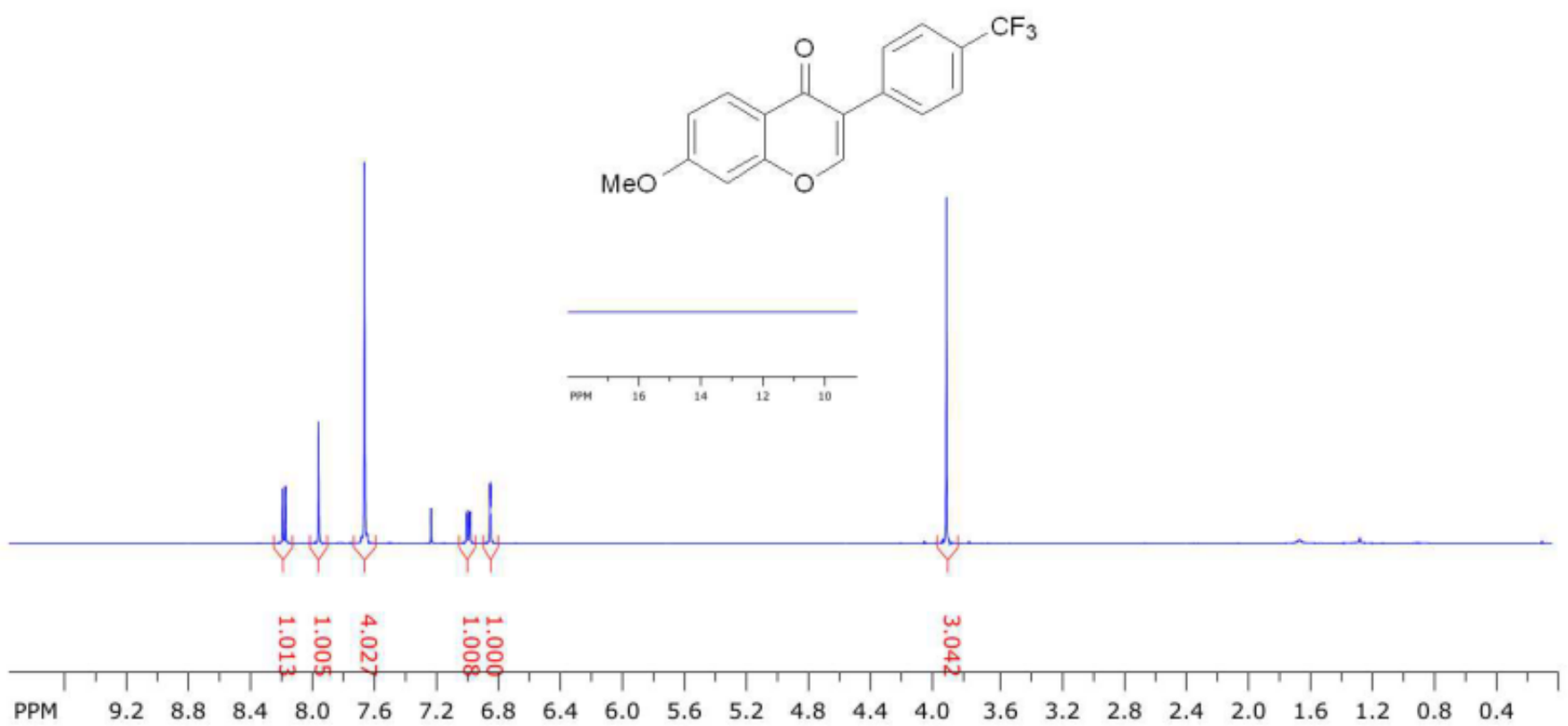

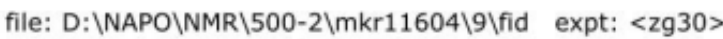

transmitter freq.: $500.133001 \mathrm{MHz}$

time domain size: 65536 points

freq. of 0 ppm: $500.130023 \mathrm{MHz}$

processed size: 65536 complex points

width: $12335.53 \mathrm{~Hz}=24.6645 \mathrm{ppm}=0.188225 \mathrm{~Hz} / \mathrm{pt}$

LB: 0.300 GF: 0.0000

number of scans: 24

$\mathrm{Hz} / \mathrm{cm}: 199.770 \mathrm{ppm} / \mathrm{cm}: 0.39943$ 


\section{Compound 5j (NMR in $\mathrm{CDCl}_{3}$ )}

SpinWorks 4: ISM $26913 \mathrm{C}$
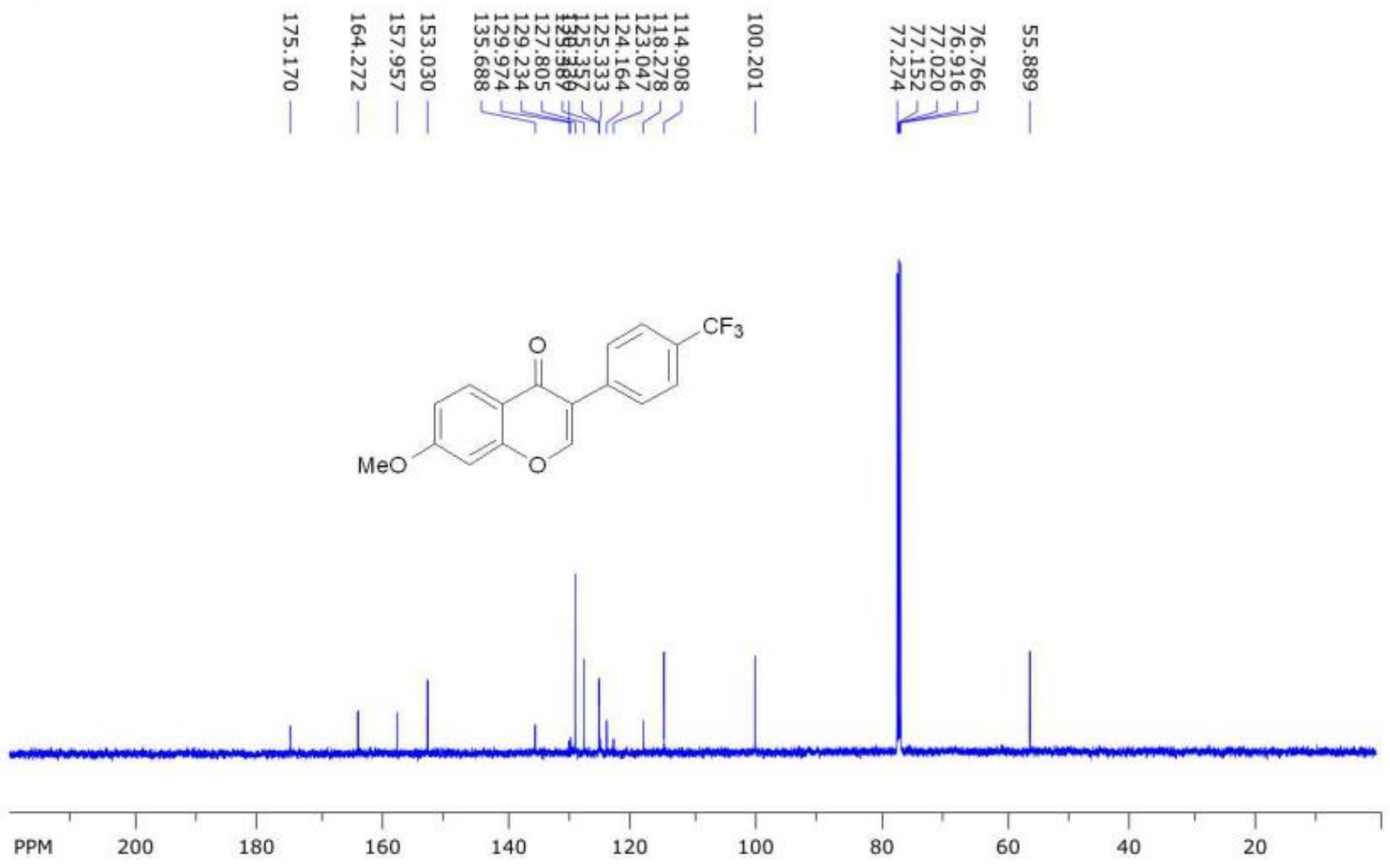

file: D:\NAPO\NMR $500-2 \backslash m k r 11604 \backslash 10 \backslash f i d$ expt: <zgpg30> transmitter freq.: $125.772879 \mathrm{MHz}$

time domain size: 65536 points

freq. of 0 ppm: $125.757792 \mathrm{MHz}$

width: $36057.69 \mathrm{~Hz}=286.6889 \mathrm{ppm}=0.550197 \mathrm{~Hz} / \mathrm{pt}$

processed size: 32768 complex points

LB: 2.000 GF: 0.0000

$\mathrm{Hz} / \mathrm{cm}: 1108.854 \mathrm{ppm} / \mathrm{cm}: 8.81632$

number of scans: 512 


\section{Compound 5k (NMR in $\mathrm{CDCl}_{3}$ )}

SpinWorks 4: ISM 68

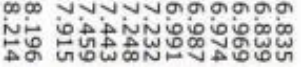

ilu
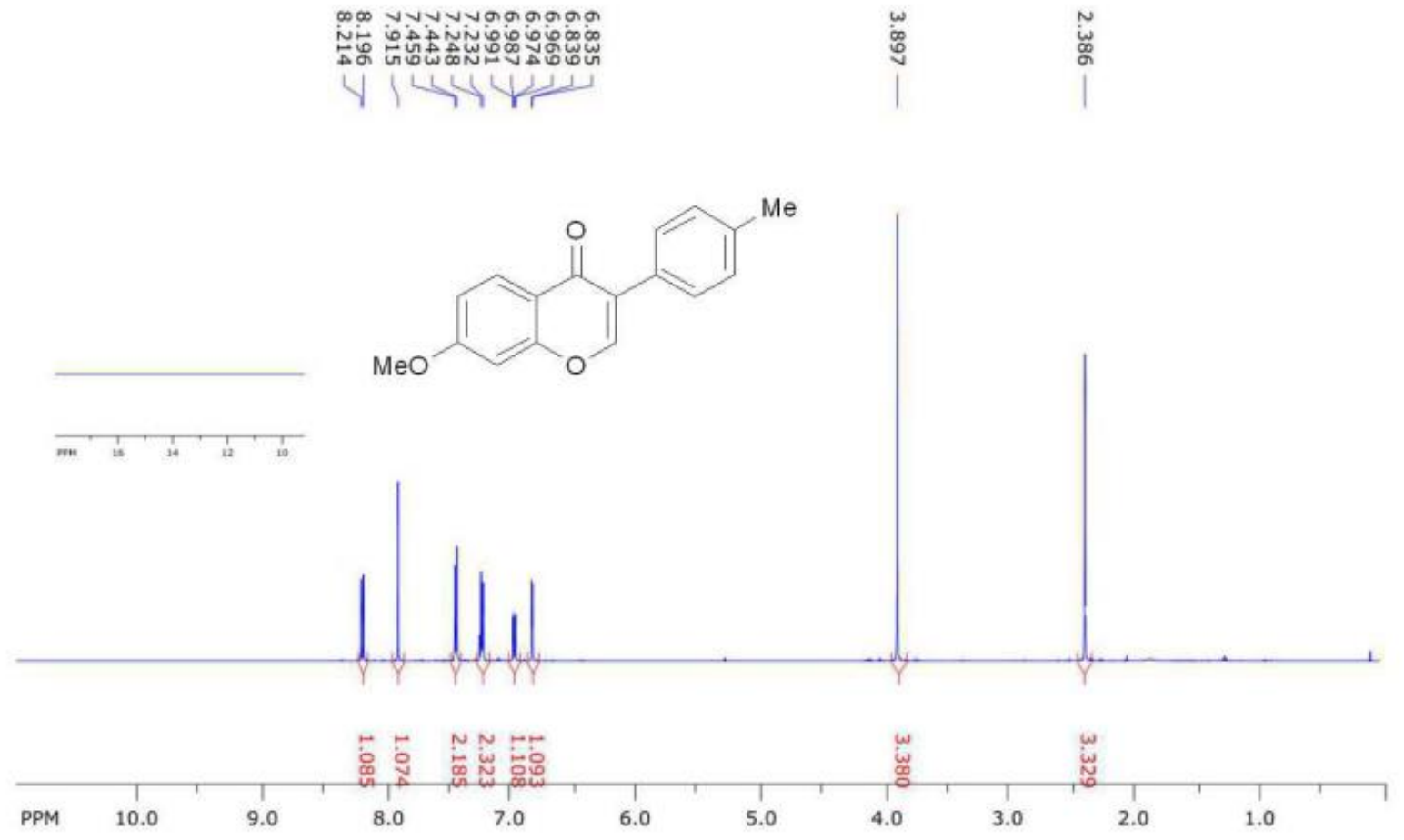

file: $F$ : $\mid$ Napo $\backslash N M R \backslash 500-1 \backslash m k r 10506 \backslash 5 \backslash$ fid expt: $<z 930\rangle$ transmitter freq.: $500.133001 \mathrm{MHz}$

time domain size: 65536 points

freq. of 0 ppm: $500.130023 \mathrm{MHz}$

processed size: 65536 complex points

LB: 0.300 GF: 0.0000

$\mathrm{Hz} / \mathrm{cm}: 219.727 \mathrm{ppm} / \mathrm{cm}: 0.43934$ 
Compound 5k (NMR in $\left.\mathrm{CDCl}_{3}\right)$

SpinWorks 4: ISM 68

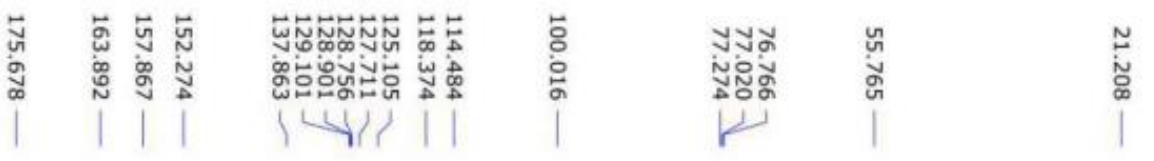

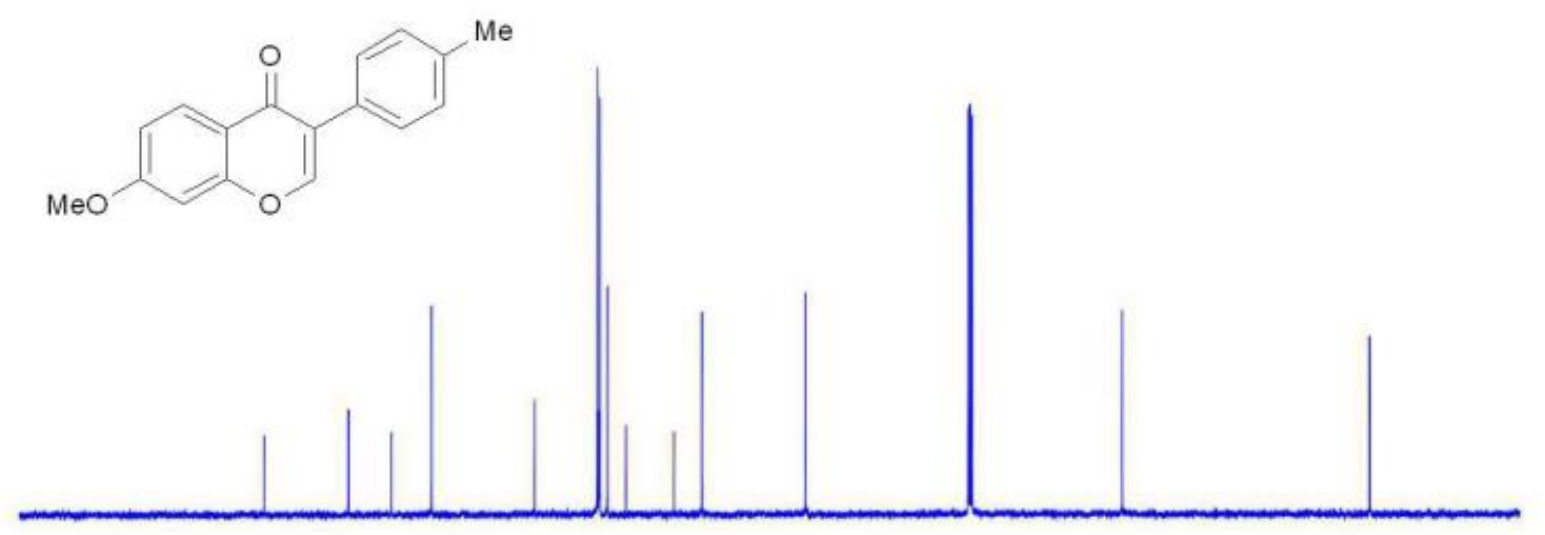

PPM

180

160

140

120

100

80

60

40

20

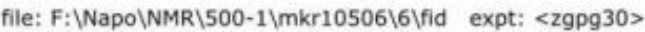

transmitter freq.: $125.772879 \mathrm{MHz}$

freq. of 0 ppm: $125.757800 \mathrm{MHz}$

processed size: 32768 complex points

time domain size: 65536 points

LB: 2.000 GF: 0.0000

$\mathrm{Hz} / \mathrm{cm}: 1054.366 \mathrm{ppm} / \mathrm{cm}: 8.38309$

width: $36057,69 \mathrm{~Hz}=286,6889 \mathrm{ppm}=0.550197 \mathrm{~Hz} / \mathrm{pt}$

$\mathrm{Hz} / \mathrm{cm}: 1054$

number of scans: 512 


\section{Compound 51 (NMR in DMSO-d ) $^{2}$}

SpinWorks 4: ISM 91-2 1H
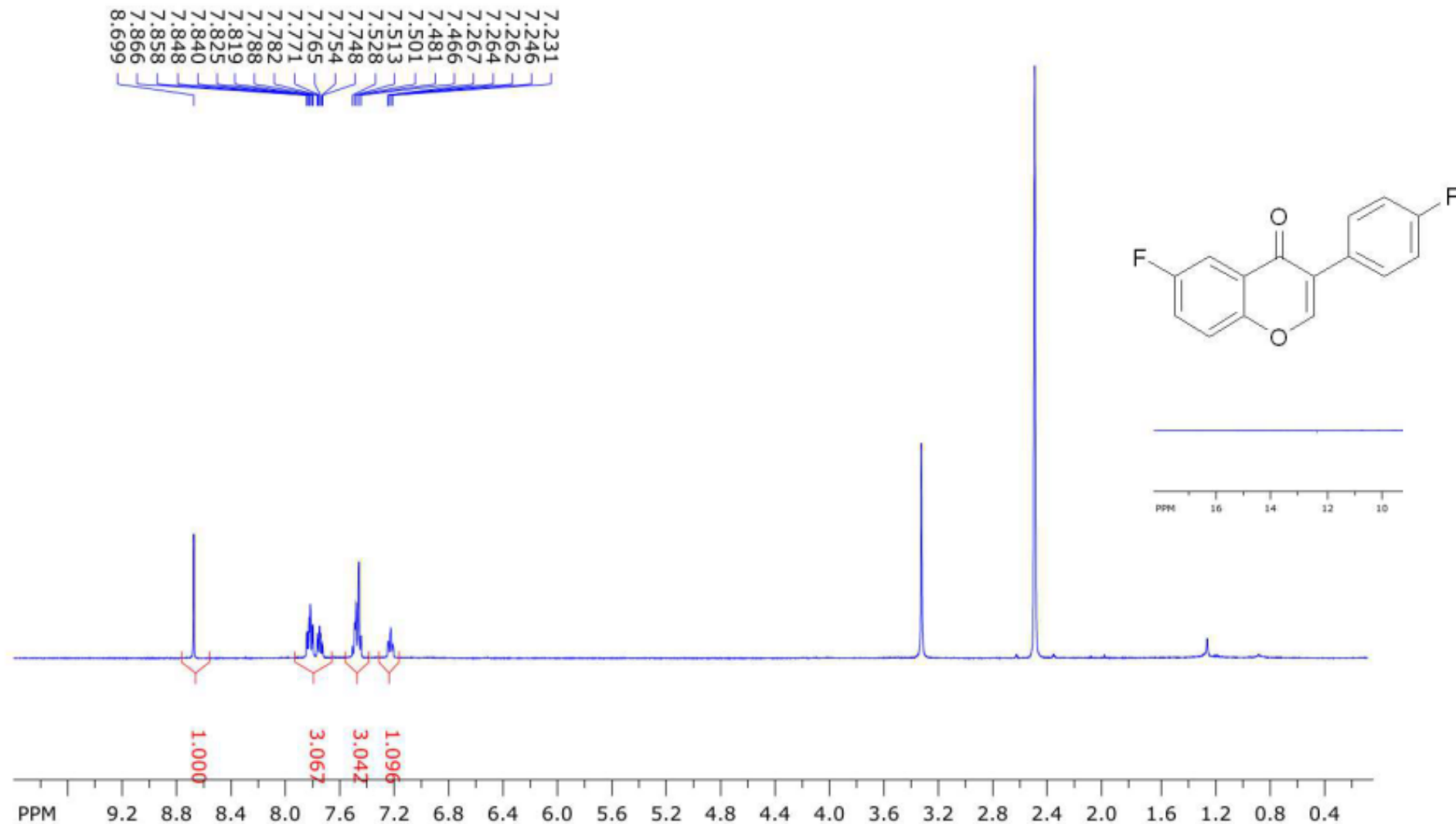

file: D:\NAPO\NMR $500-1 \backslash m k r 12611 \backslash 7 \backslash$ fid expt: <zg30> transmitter freq.: $500.133001 \mathrm{MHz}$

time domain size: 65536 points

freq. of $0 \mathrm{ppm}$ : $500.130005 \mathrm{MHz}$

processed size: 65536 complex points

width: $12335.53 \mathrm{~Hz}=24.6645 \mathrm{ppm}=0.188225 \mathrm{~Hz} / \mathrm{pt}$

LB: 0.300 GF: 0.0000

number of scans: 24

$\mathrm{Hz} / \mathrm{cm}: 199.770 \mathrm{ppm} / \mathrm{cm}: 0.39943$ 


\section{Compound 5m (NMR in $\left.\mathrm{CDCl}_{3}\right)$}

SpinWorks 4: ISM $2891 \mathrm{H}$
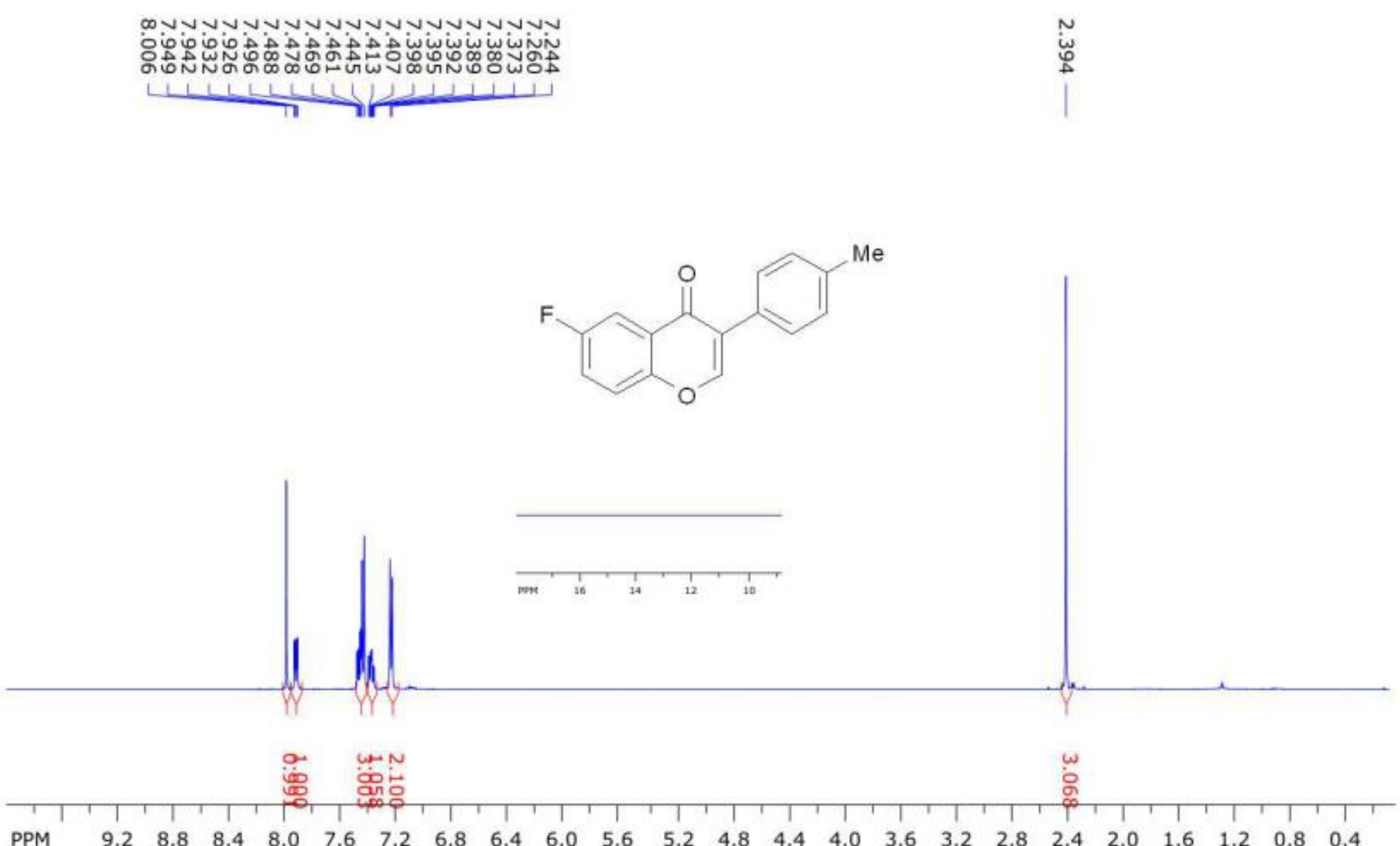

file: D: \NAPO \NMR $\backslash 500-2 \backslash m k r 11604 \backslash 7 \backslash$ fid expt: <zg30> transmitter freq.: $500.133001 \mathrm{MHz}$

time domain size: 65536 points

width: $12335.53 \mathrm{~Hz}=24.6645 \mathrm{ppm}=0.188225 \mathrm{~Hz} / \mathrm{pt}$

freq. of 0 ppm: $500.130022 \mathrm{MHz}$

processed size: 65536 complex points

LB: 0.300 GF: 0.0000

$\mathrm{Hz} / \mathrm{cm}: 199.224 \mathrm{ppm} / \mathrm{cm}: 0.39834$

number of scans: 24 


\section{Compound 5m (NMR in $\left.\mathrm{CDCl}_{3}\right)$}

SpinWorks 4: ISM 289 13C
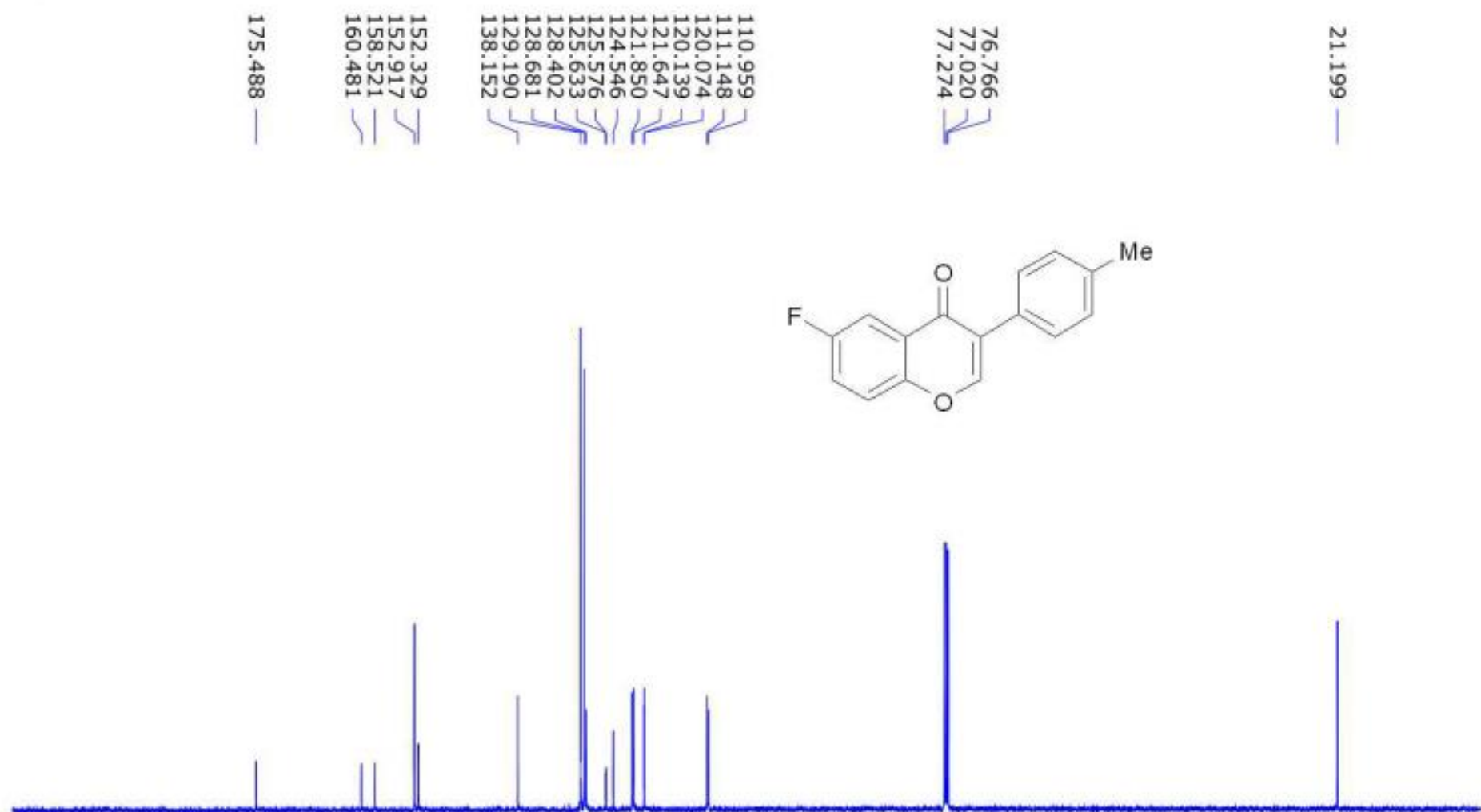

PPM

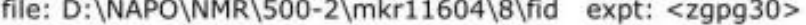
transmitter freq.: $125.772879 \mathrm{MHz}$

time domain size: 65536 points

width: $36057.69 \mathrm{~Hz}=286.6889 \mathrm{ppm}=0.550197 \mathrm{~Hz} / \mathrm{pt}$

number of scans: 512 freq of 0 ppm: $125,757802 \mathrm{MHz}$

processed size: 32768 complex points

LB: 2.000 GF: 0.0000

$\mathrm{Hz} / \mathrm{cm}: 1054.608 \mathrm{ppm} / \mathrm{cm}: 8.38502$ 


\section{Compound 5n (NMR in $\left.\mathrm{CDCl}_{3}\right)$}

SpinWorks 4: ISM 119 1H

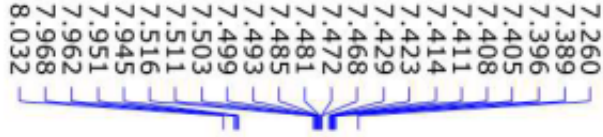

Tr
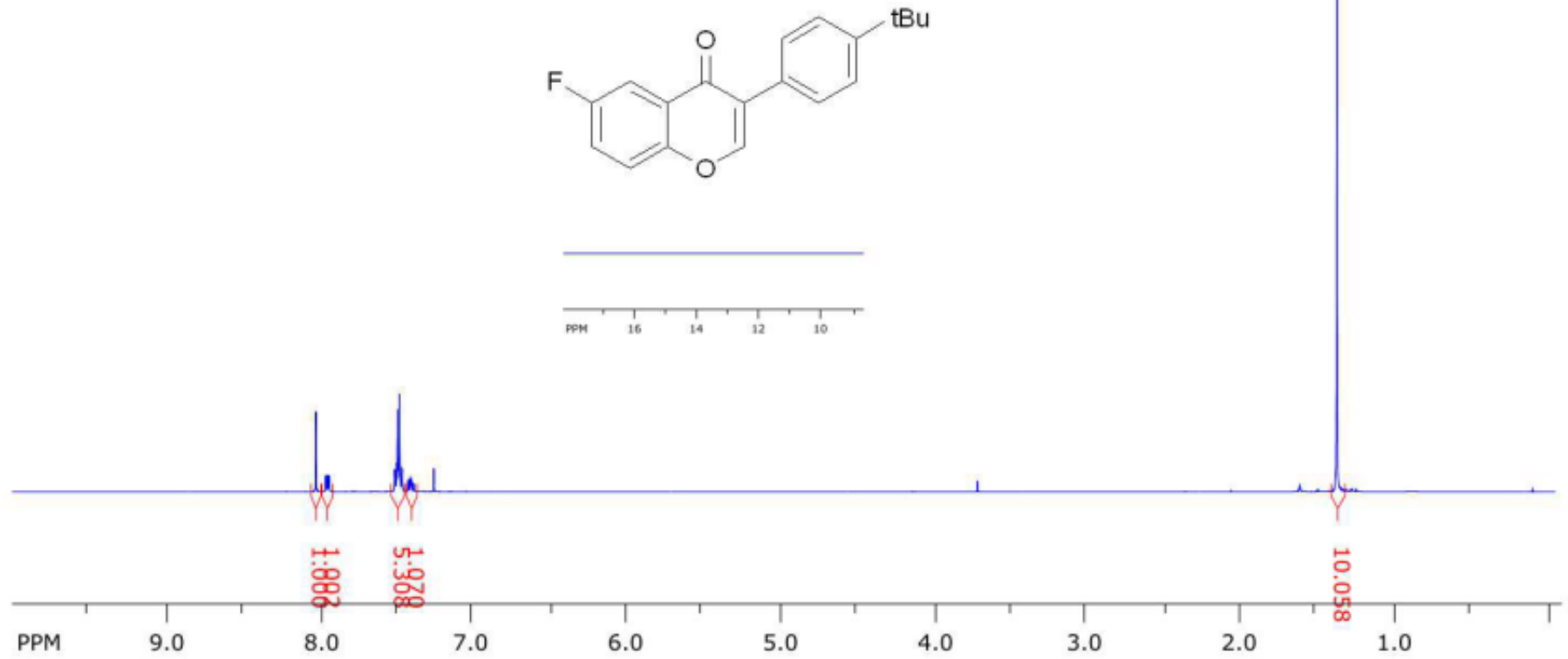

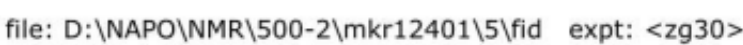

freq. of $0 \mathrm{ppm}$ : $500.130023 \mathrm{MHz}$

transmitter freq.: $500.133001 \mathrm{MHz}$

time domain size: 65536 points

processed size: 65536 complex points

width: $12335.53 \mathrm{~Hz}=24.6645 \mathrm{ppm}=0.188225 \mathrm{~Hz} / \mathrm{pt}$

LB: 0.300 GF: 0.0000

$\mathrm{Hz} / \mathrm{cm}: 201.953 \mathrm{ppm} / \mathrm{cm}: 0.40380$

number of scans: 24 


\section{Compound 5n (NMR in $\left.\mathrm{CDCl}_{3}\right)$}

SpinWorks 4: ISM 119 13C

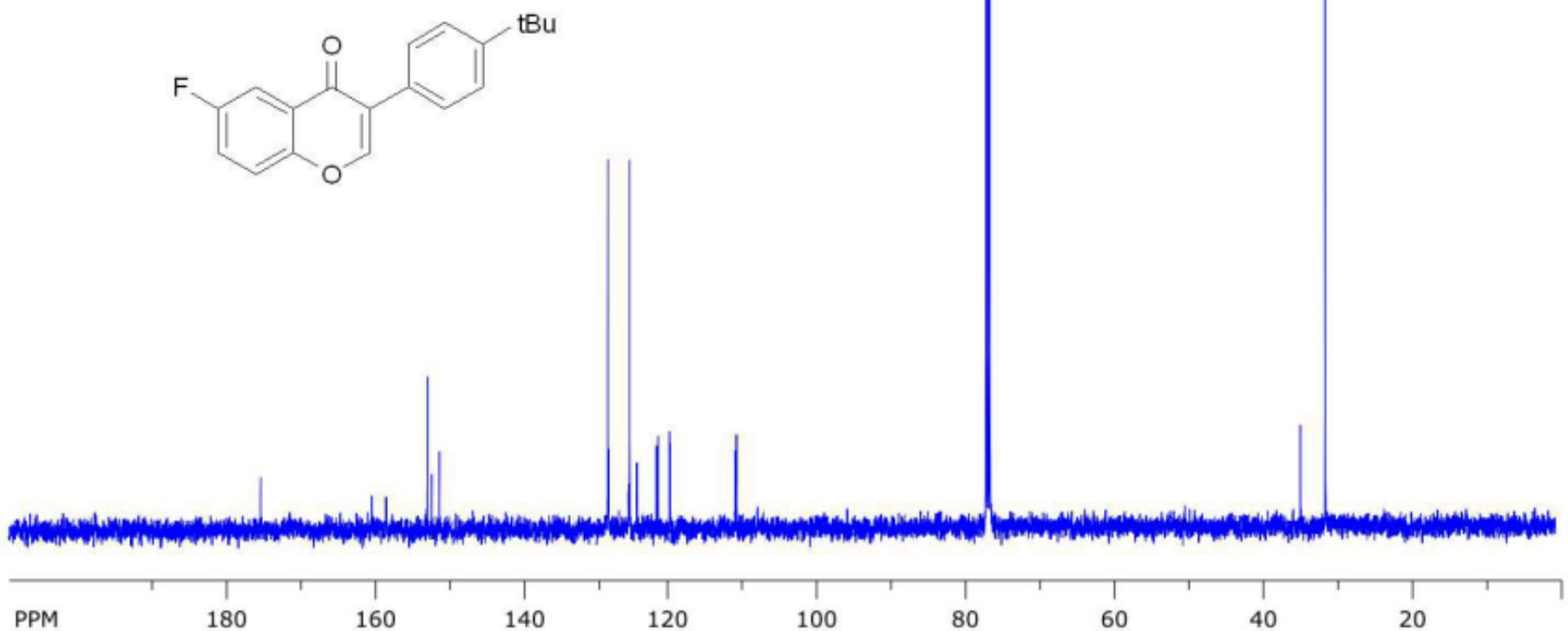

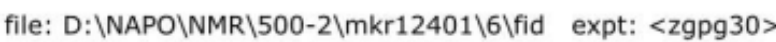
transmitter freq.: $125.772879 \mathrm{MHz}$

freq. of $0 \mathrm{ppm}$ : $125.757792 \mathrm{MHz}$

time domain size: 65536 points

processed size: 32768 complex points

B: 2.000 GF: 0.0000

width: $36057.69 \mathrm{~Hz}=286.6889 \mathrm{ppm}=0.550197 \mathrm{~Hz} / \mathrm{pt}$

$\mathrm{Hz} / \mathrm{cm}: 1054.608 \mathrm{ppm} / \mathrm{cm}: 8.38502$

number of scans: 256 


\section{Compound 5 o (NMR in $\left.\mathrm{CDCl}_{3}\right)$}

SpinWorks 4: ISM 307 1H
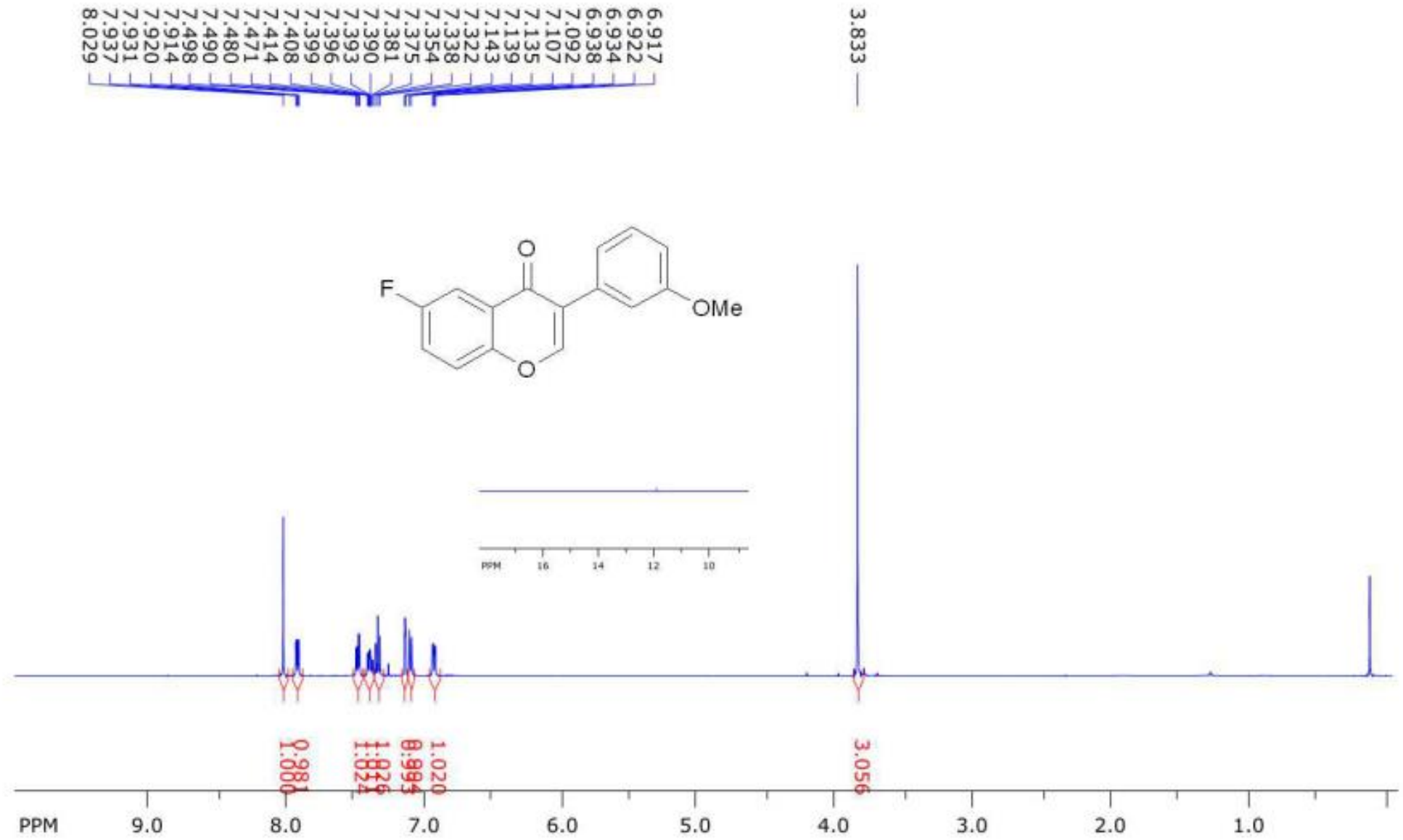

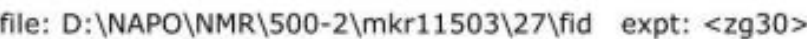
transmitter freq.: $500.133001 \mathrm{MHz}$

time domain size: 65536 points

freq. of 0 ppm: $500.130023 \mathrm{MHz}$

processed size: 65536 complex points

LB: 0.300 GF: 0.0000

width: $12335.53 \mathrm{~Hz}=24.6645 \mathrm{ppm}=0.188225 \mathrm{~Hz} / \mathrm{pt}$

$\mathrm{Hz} / \mathrm{cm}: 201.407 \mathrm{ppm} / \mathrm{cm}: 0.40271$ 


\section{Compound 5 o (NMR in $\left.\mathrm{CDCl}_{3}\right)$}

SpinWorks 4: ISM 307 13C
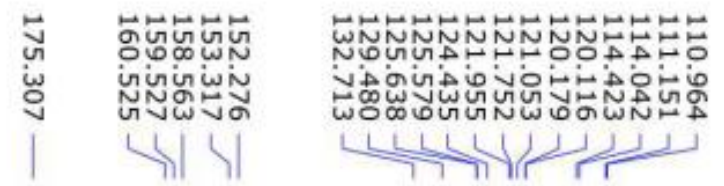<smiles>COc1cccc(-c2coc3ccc(F)cc3c2=O)c1</smiles>

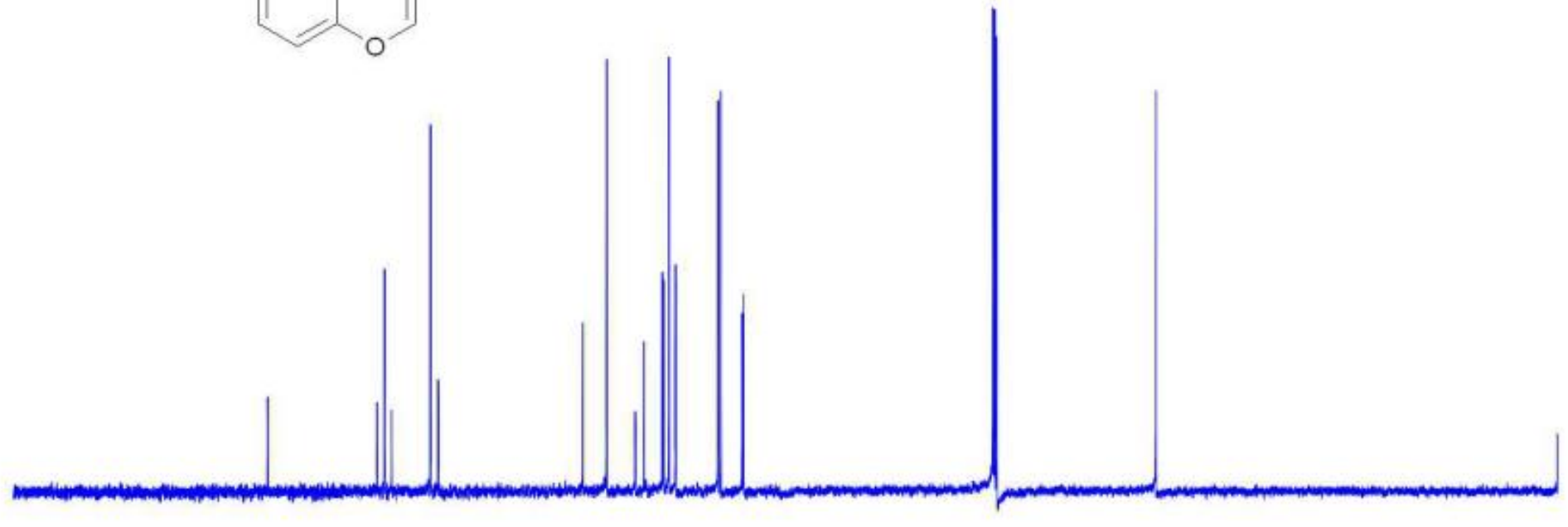

PPM

180

160

140

120

100

80

60

40

20

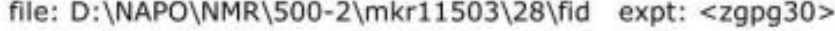
transmitter freq.: $125.772879 \mathrm{MHz}$

time domain size: 65536 points

width: $36057.69 \mathrm{~Hz}=286.6889 \mathrm{ppm}=0.550197 \mathrm{~Hz} / \mathrm{pt}$ number of scans: 512 freq. of 0 ppm: $125.757802 \mathrm{MHz}$

processed size: 32768 complex points

LB: 2.000 GF: 0.0000

$\mathrm{Hz} / \mathrm{cm}$ : $1049.821 \quad \mathrm{ppm} / \mathrm{cm}: 8.34696$ 


\section{Compound 5p (NMR in $\mathrm{CDCl}_{3}$ )}

SpinWorks 4: ISM 102 1H
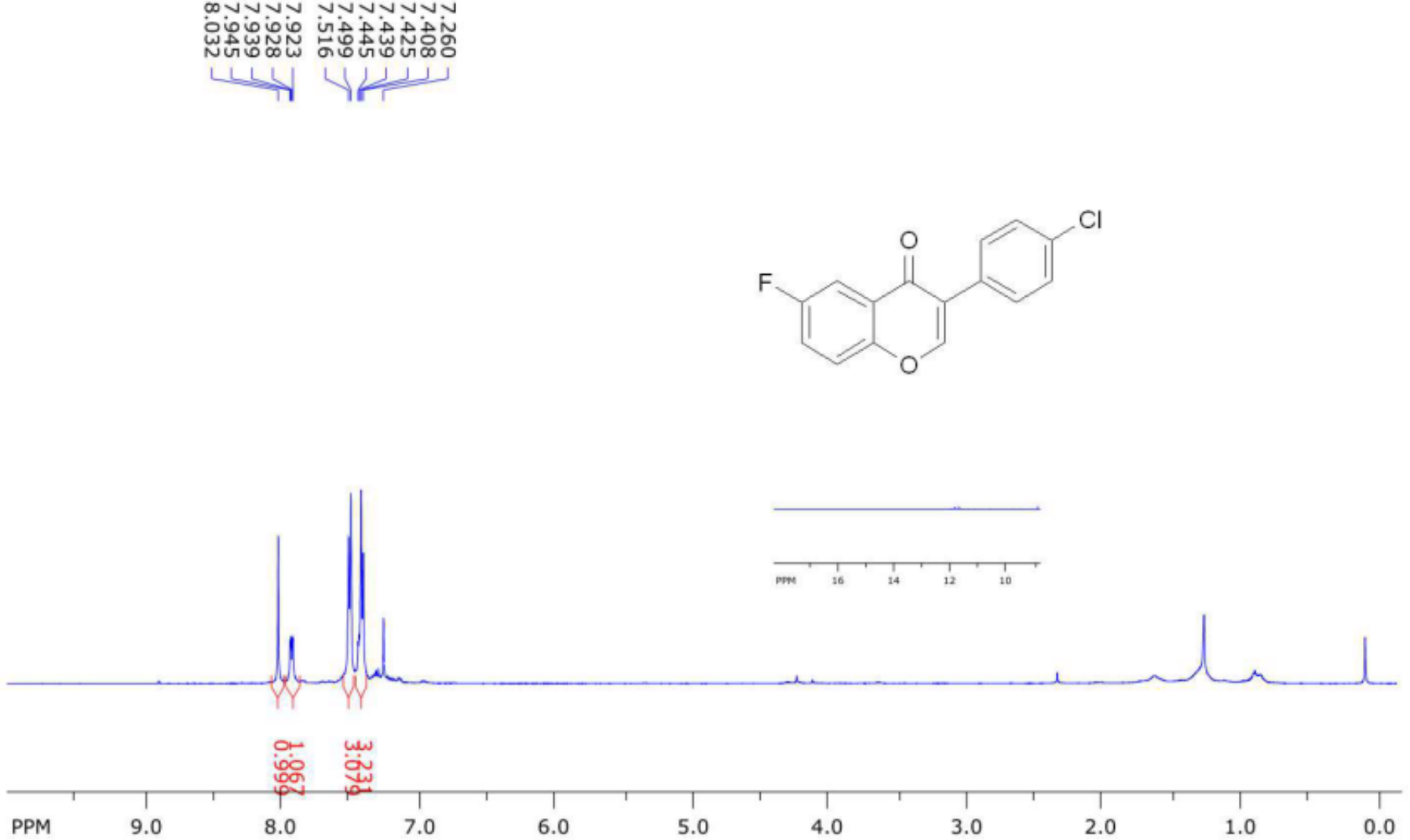

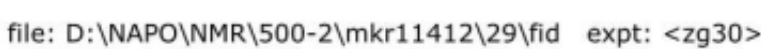

freq. of 0 ppm: $500.130023 \mathrm{MHz}$

transmitter freq.: $500.133001 \mathrm{MHz}$

time domain size: 65536 points

processed size: 65536 complex points

width: $12335.53 \mathrm{~Hz}=24.6645 \mathrm{ppm}=0.188225 \mathrm{~Hz} / \mathrm{pt}$

LB: 0.300 GF: 0.0000

$\mathrm{Hz} / \mathrm{cm}: 203.591 \mathrm{ppm} / \mathrm{cm}: 0.40707$

number of scans: 24 


\section{Compound 5p (NMR in $\left.\mathrm{CDCl}_{3}\right)$}

SpinWorks 4: ISM 102 13C
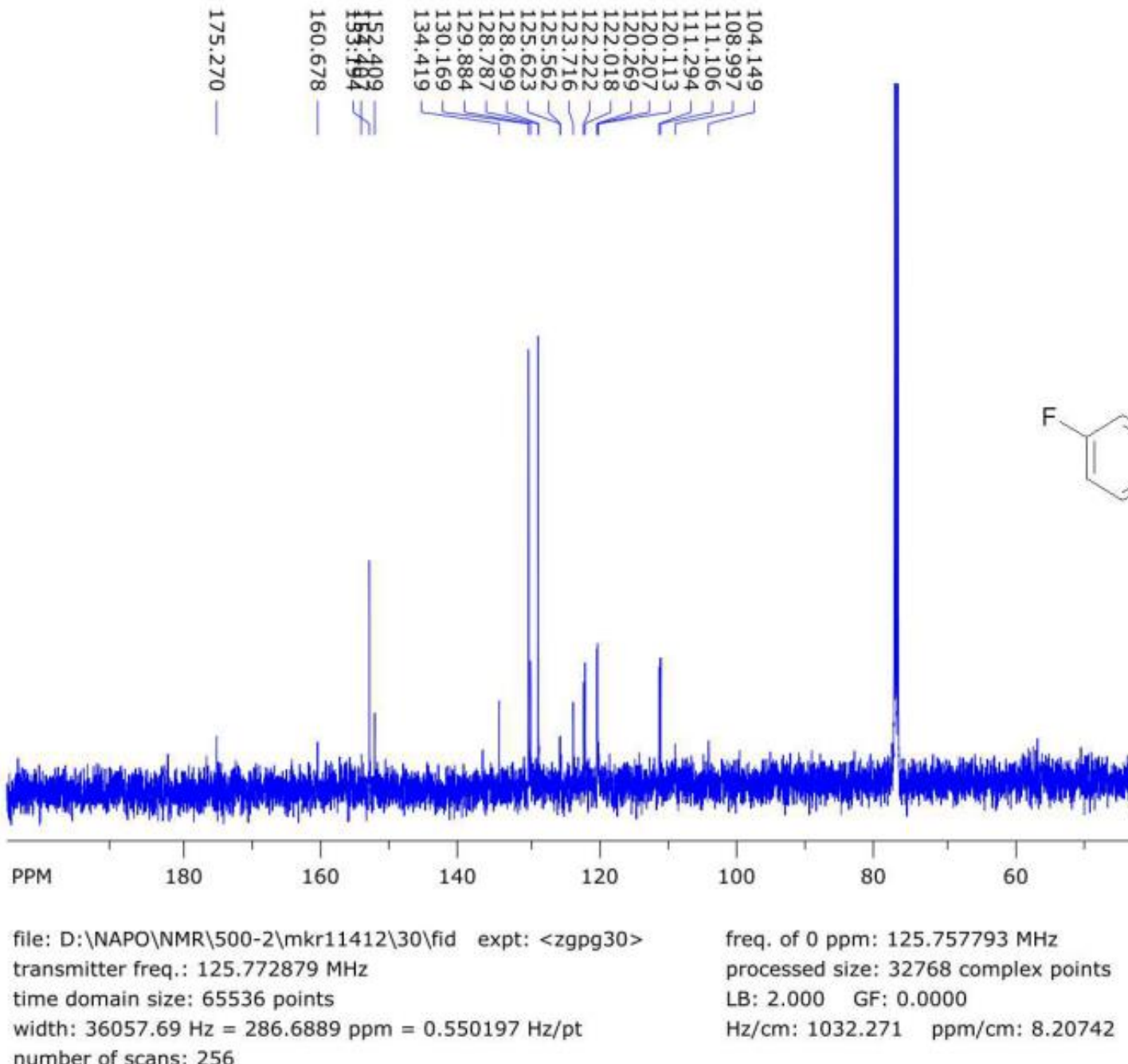

number of scans: 256 


\section{Compound 5q (NMR in $\mathrm{CDCl}_{3}$ )}

SpinWorks 4: ISM $1201 \mathrm{H}$

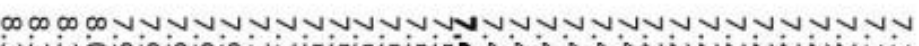
W.
WN ᄀ 117 1

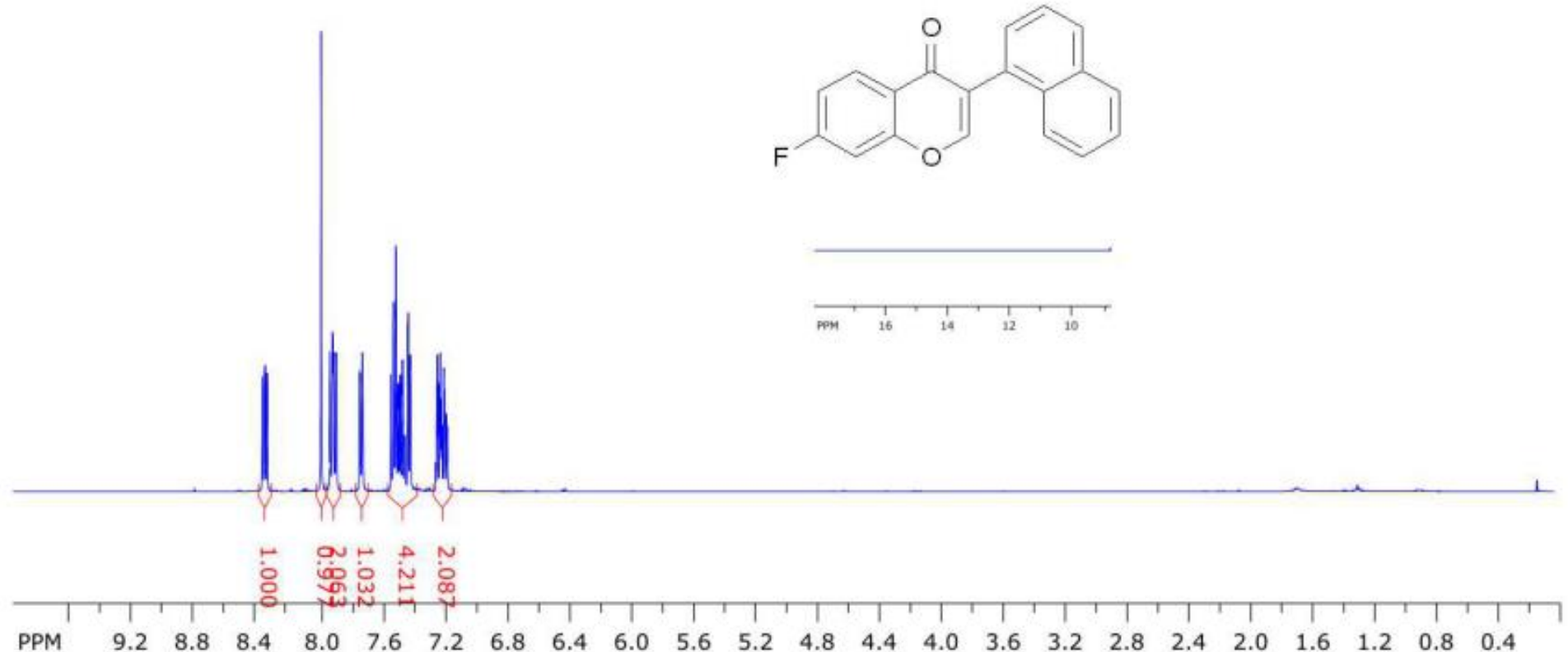

8.07 .6

freq. of 0 ppm: $500.130023 \mathrm{MHz}$

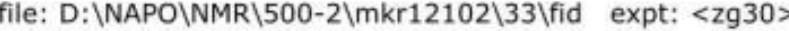

processed size: 65536 complex points

transmitter freq.: $500.133001 \mathrm{MHz}$

time domain size: 65536 points

LB: 0.300 GF: 0.0000

width: $12335.53 \mathrm{~Hz}=24.6645 \mathrm{ppm}=0.188225 \mathrm{~Hz} / \mathrm{pt}$

$\mathrm{Hz} / \mathrm{cm}: 199.224 \mathrm{ppm} / \mathrm{cm}: 0.39834$

number of scans: 24 


\section{Compound 5q (NMR in $\left.\mathrm{CDCl}_{3}\right)$}

SpinWorks 4: ISM 120 13C
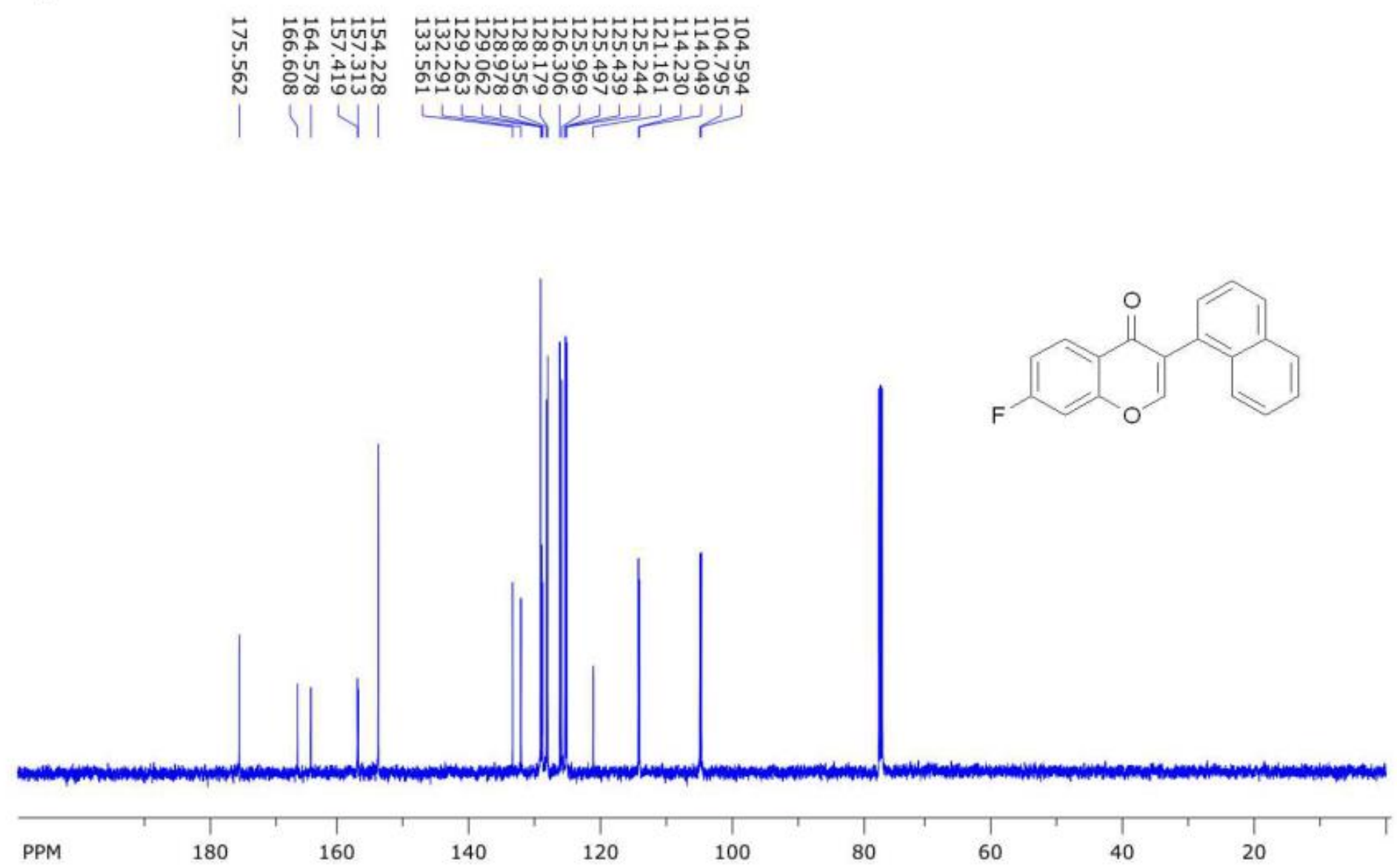

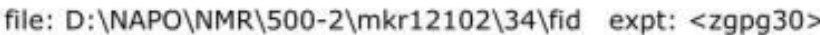
transmitter freq.: $125.772879 \mathrm{MHz}$

time domain size: 65536 points

freq. of 0 ppm: $125.757807 \mathrm{MHz}$

width: $36057.69 \mathrm{~Hz}=286.6889 \mathrm{ppm}=0.550197 \mathrm{~Hz} / \mathrm{pt}$ processed size: 32768 complex points

LB: 2.000 GF: 0.0000

number of scans: 164

$\mathrm{Hz} / \mathrm{cm}: 1057.799 \mathrm{ppm} / \mathrm{cm}: 8.41039$ 


\section{Compound 5r (NMR in $\left.\mathrm{CDCl}_{3}\right)$}

SpinWorks 4: ISM $2271 \mathrm{H}$
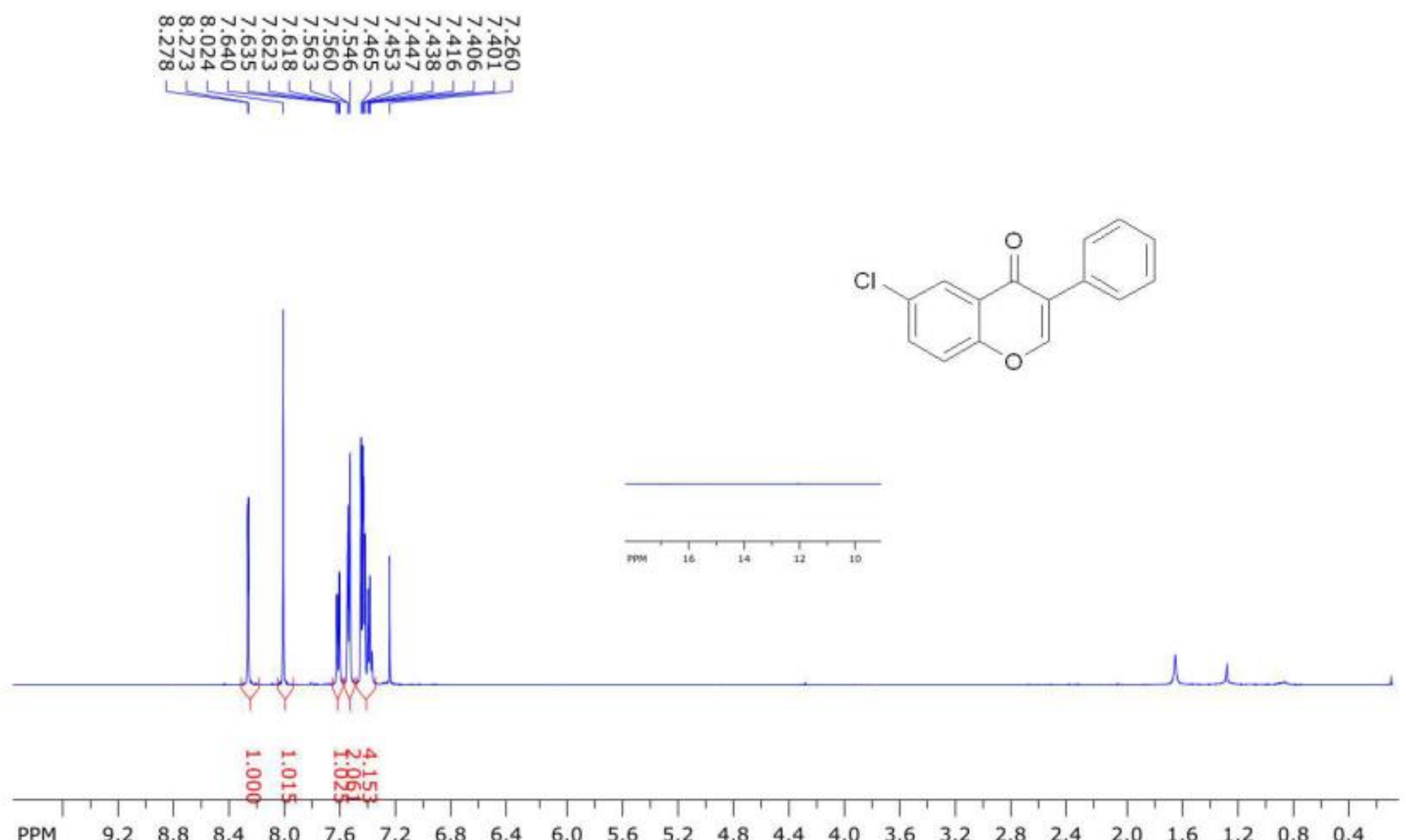

file: D: \NAPO\NMR $500-1 \backslash m k r 10509 \backslash 1 \backslash$ fid expt: <zg30> transmitter freq.: $500.133001 \mathrm{MHz}$

time domain size: 65536 points

width: $12335.53 \mathrm{~Hz}=24.6645 \mathrm{ppm}=0.188225 \mathrm{~Hz} / \mathrm{pt}$

number of scans: 32 freq. of 0 ppm: $500.130024 \mathrm{MHz}$

processed size: 65536 complex points

LB: 0.300 GF: 0.0000

$\mathrm{Hz} / \mathrm{cm}: 198.133 \mathrm{ppm} / \mathrm{cm}: 0.39616$ 


\section{Compound 5r (NMR in $\left.\mathrm{CDCl}_{3}\right)$}

SpinWorks 4: ISM $22713 \mathrm{C}$
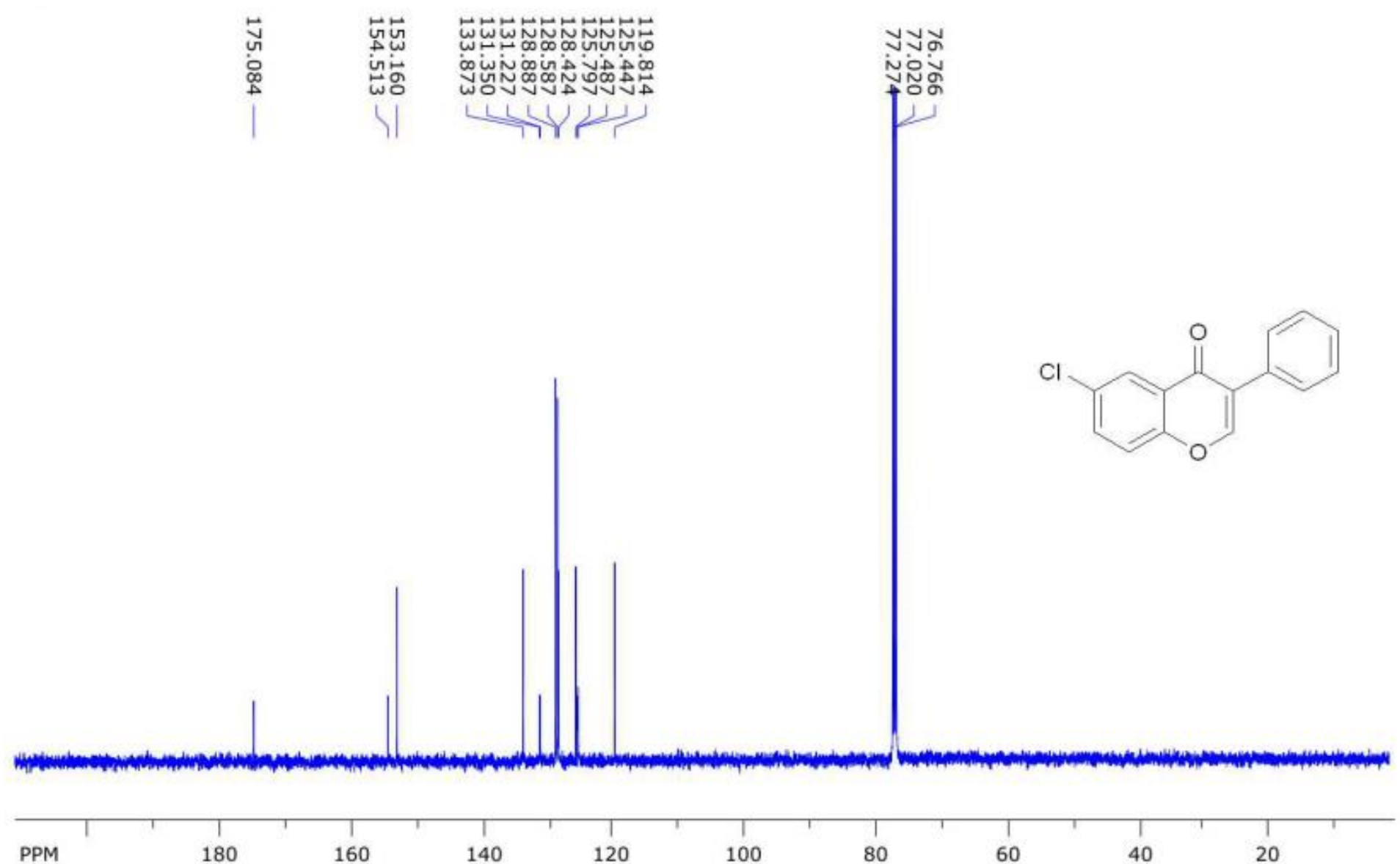

100

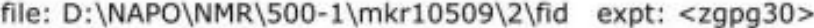
transmitter freq.: $125.772879 \mathrm{MHz}$

time domain size: 65536 points

width: $36057.69 \mathrm{~Hz}=286.6889 \mathrm{ppm}=0.550197 \mathrm{~Hz} / \mathrm{pt}$

number of scans: 512 freq. of $0 \mathrm{ppm}: 125.757794 \mathrm{MHz}$

processed size: 32768 complex points

LB: 2.000 GF: 0.0000

$\mathrm{Hz} / \mathrm{cm}: 1057.799 \mathrm{ppm} / \mathrm{cm}: 8.41039$ 


\section{Compound 5s (NMR in $\left.\mathrm{CDCl}_{3}\right)$}

SpinWorks 4: ISM $231 \mathrm{H}$
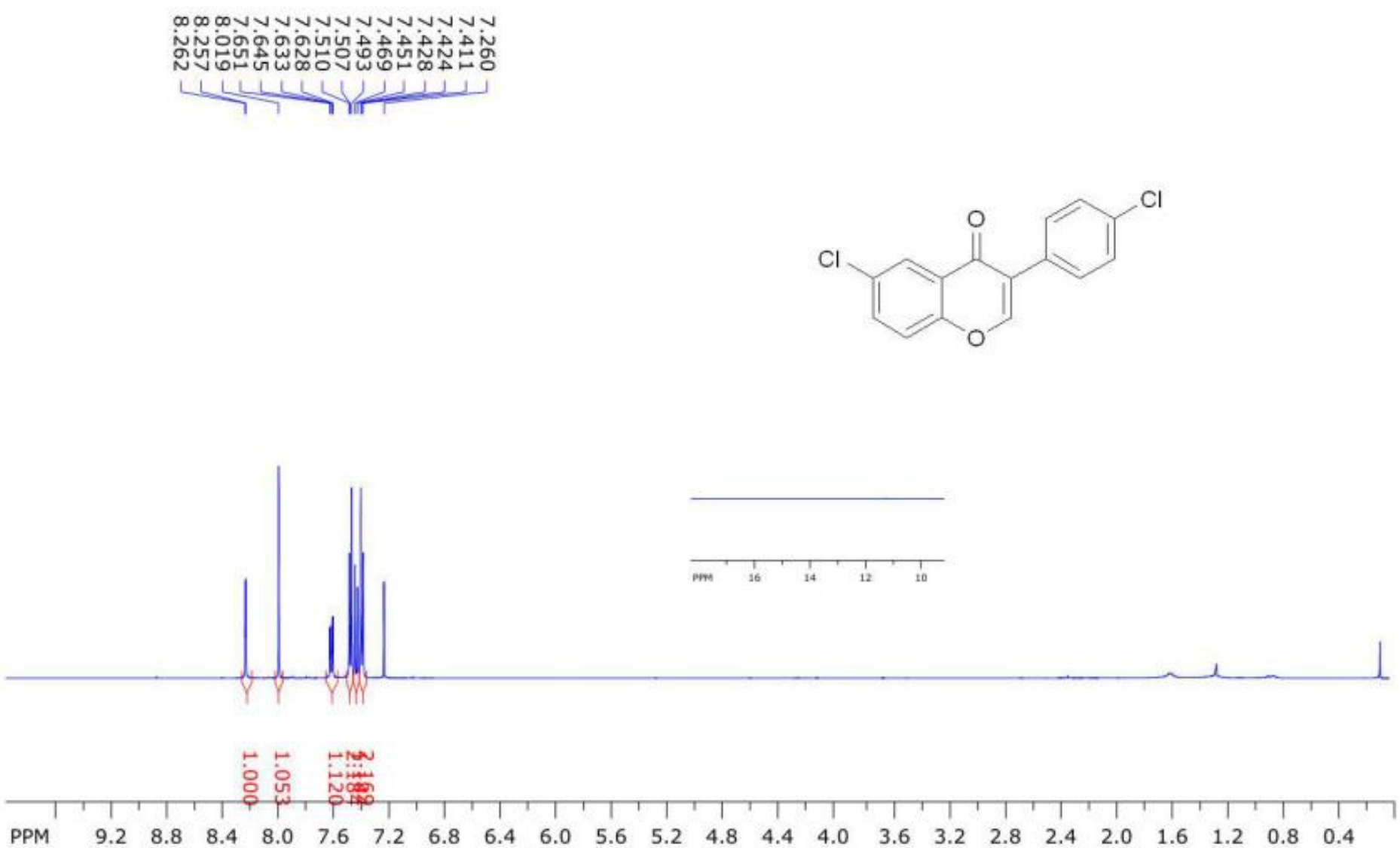

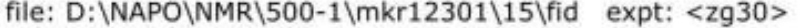
transmitter freq.: $500.133001 \mathrm{MHz}$

time domain size: 65536 points

width: $12335.53 \mathrm{~Hz}=24.6645 \mathrm{ppm}=0.188225 \mathrm{~Hz} / \mathrm{pt}$ number of scans: 24 freq. of $0 \mathrm{ppm}$ : $500.130023 \mathrm{MHz}$

processed size: 65536 complex points

LB: 0.300 GF: 0.0000

$\mathrm{Hz} / \mathrm{cm}: 199.770 \mathrm{ppm} / \mathrm{cm}: 0.39943$ 


\section{Compound 5s (NMR in $\left.\mathrm{CDCl}_{3}\right)$}

SpinWorks 4: ISM $2313 \mathrm{C}$
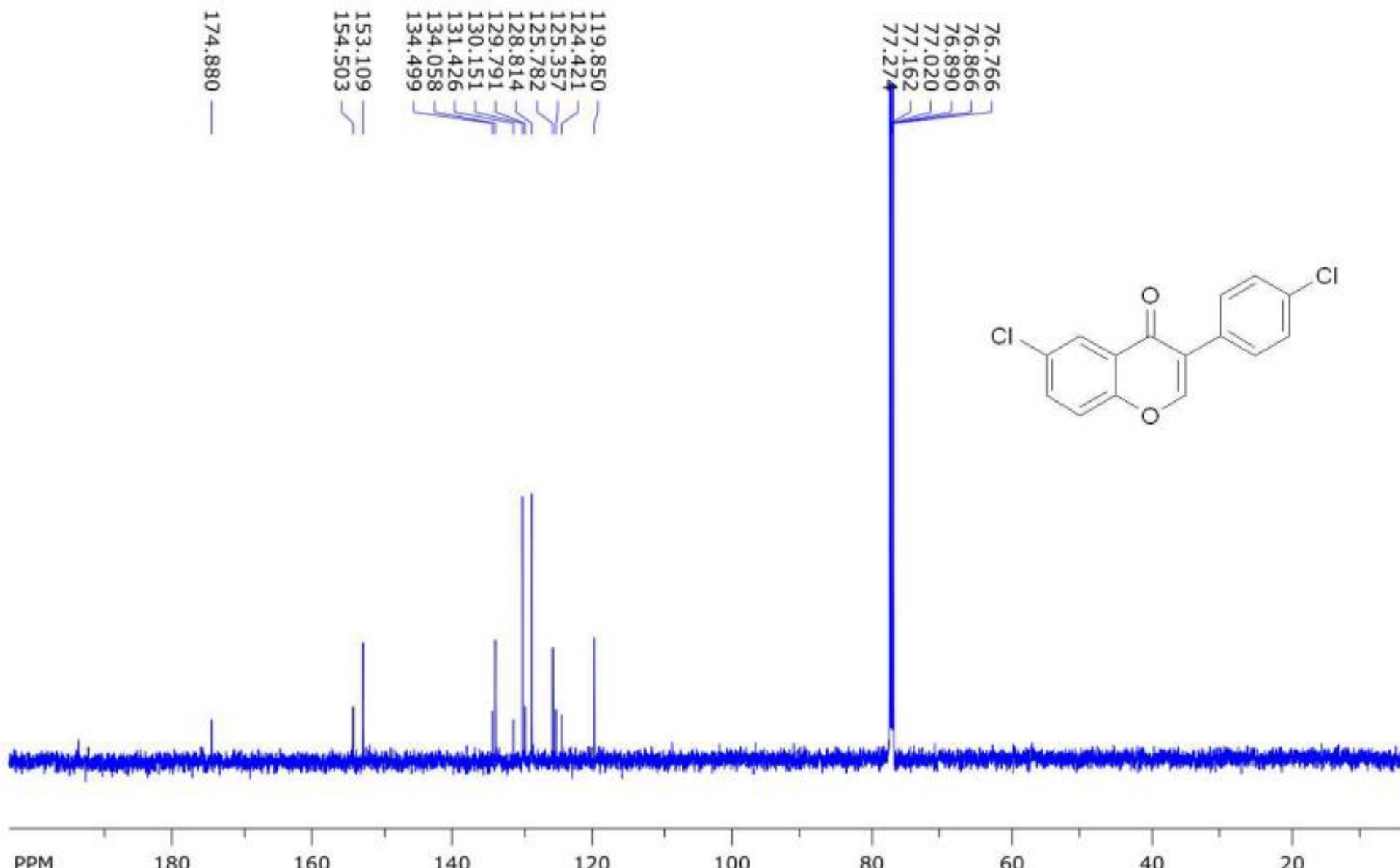

140

120

100

80

60

40

20

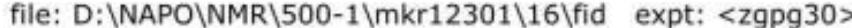
transmitter freq.: $125.772879 \mathrm{MHz}$

time domain size: 65536 points

width: $36057.69 \mathrm{~Hz}=286.6889 \mathrm{ppm}=0.550197 \mathrm{~Hz} / \mathrm{pt}$ number of scans: 256 freq. of $0 \mathrm{ppm}: 125.757792 \mathrm{MHz}$

processed size: 32768 complex points

LB: 2.000 GF: 0.0000

$\mathrm{Hz} / \mathrm{cm}: 1009.934 \mathrm{ppm} / \mathrm{cm}: 8.02983$ 


\section{Compound 5t (NMR in $\left.\mathrm{CDCl}_{3}\right)$}

SpinWorks 4: ISM 29
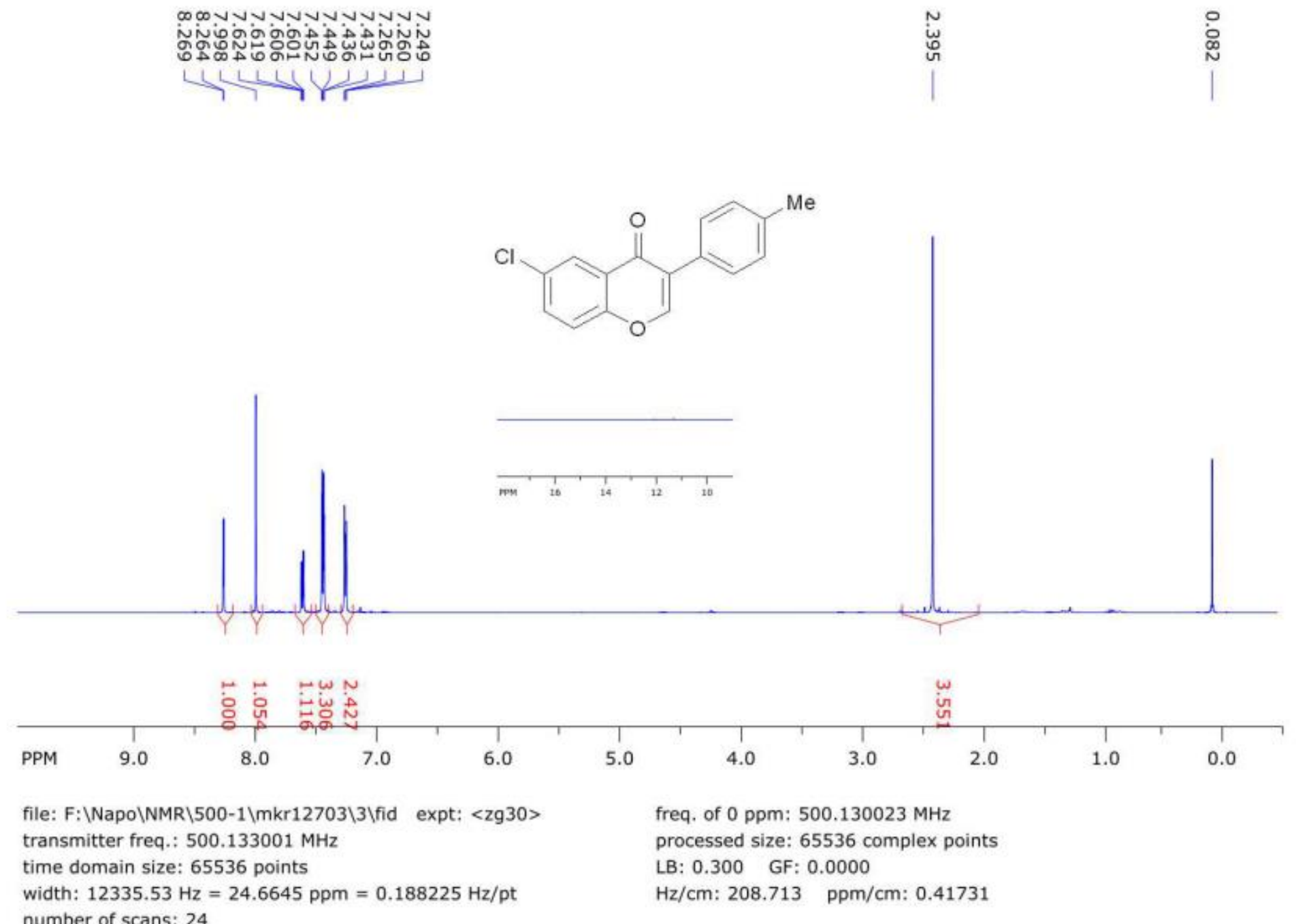


\section{Compound 5u (NMR in $\mathrm{CDCl}_{3}$ )}

SpinWorks 4: ISM 123 1H

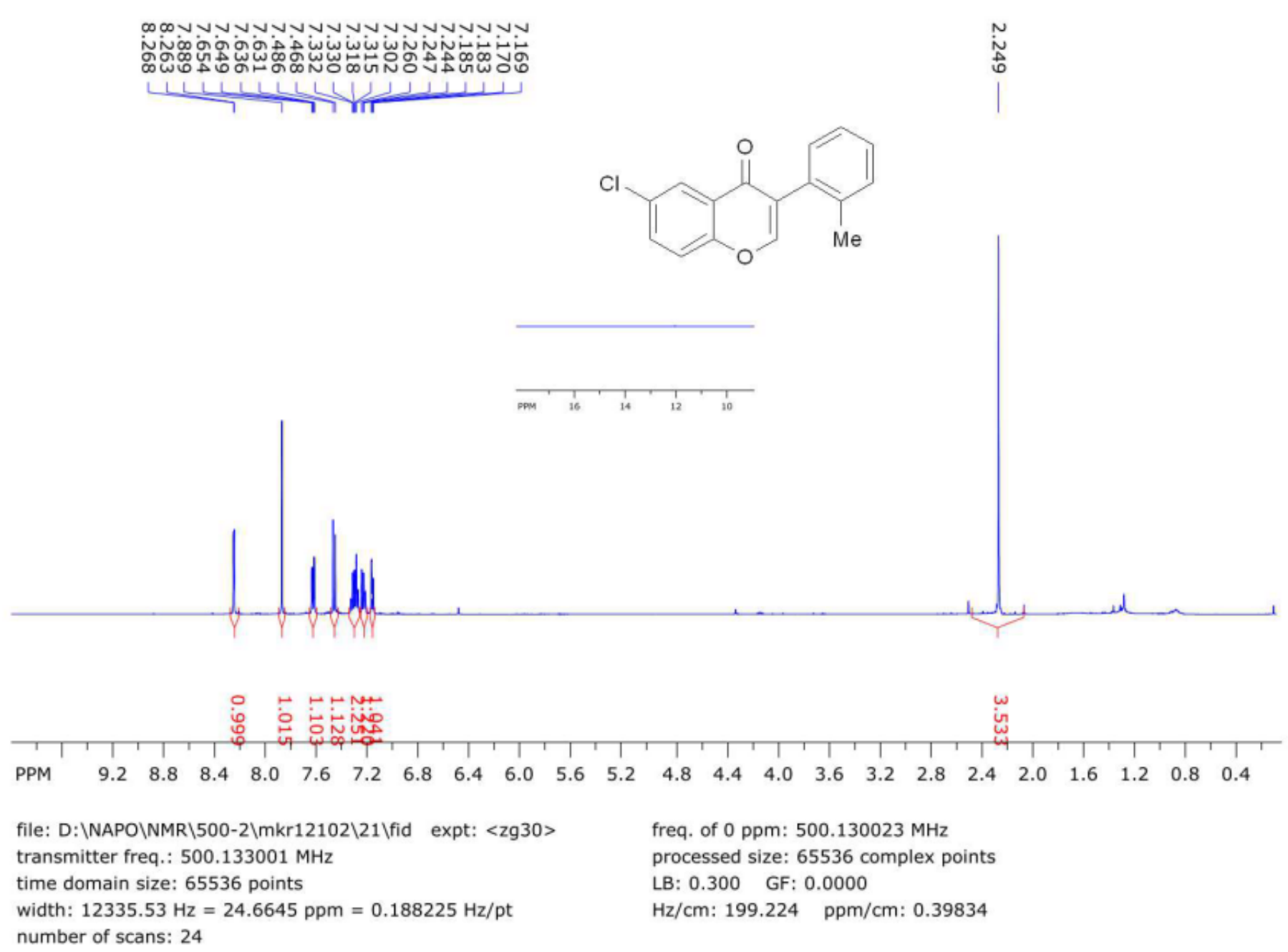




\section{Compound 5u (NMR in $\left.\mathrm{CDCl}_{3}\right)$}

SpinWorks 4: ISM 123 13C
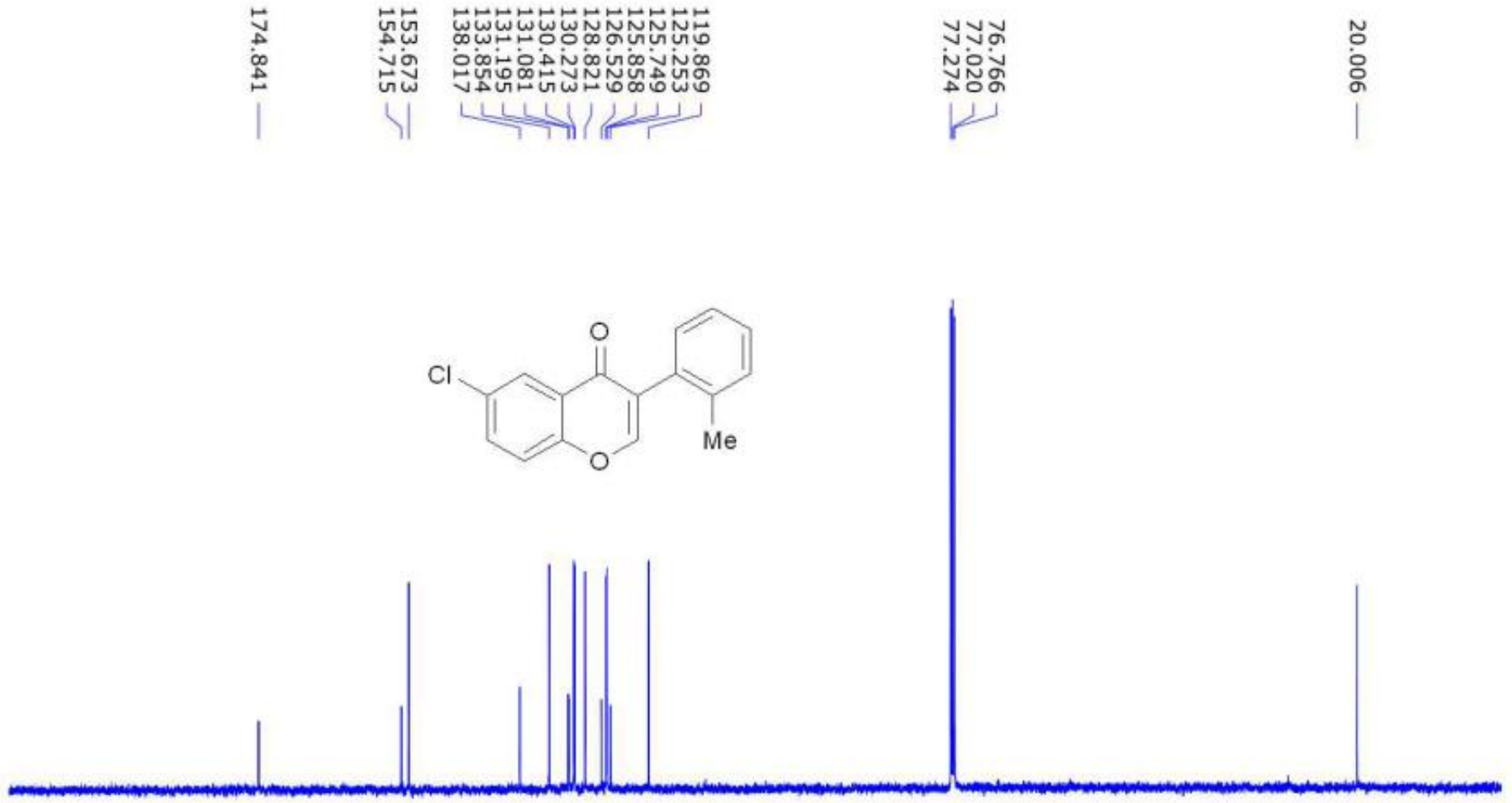

PPM

file: D: \NAPO\NMR $\backslash 500-2 \backslash m k r 12102 \backslash 22 \backslash$ fid expt: <zgpg30> transmitter freq.: $125.772879 \mathrm{MHz}$

time domain size: 65536 points

width: $36057.69 \mathrm{~Hz}=286.6889 \mathrm{ppm}=0.550197 \mathrm{~Hz} / \mathrm{pt}$ number of scans: 512 freq. of 0 ppm: $125.757796 \mathrm{MHz}$ processed size: 32768 complex points LB: 2.000 GF: 0.0000

$\mathrm{Hz} / \mathrm{cm}: 1057.799 \mathrm{ppm} / \mathrm{cm}: 8.41039$ 


\section{Compound 5v (NMR in $\left.\mathrm{CDCl}_{3}\right)$}

SpinWorks 4: ISM 108 1H

$$
\sin (\sin )
$$

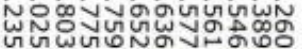

H
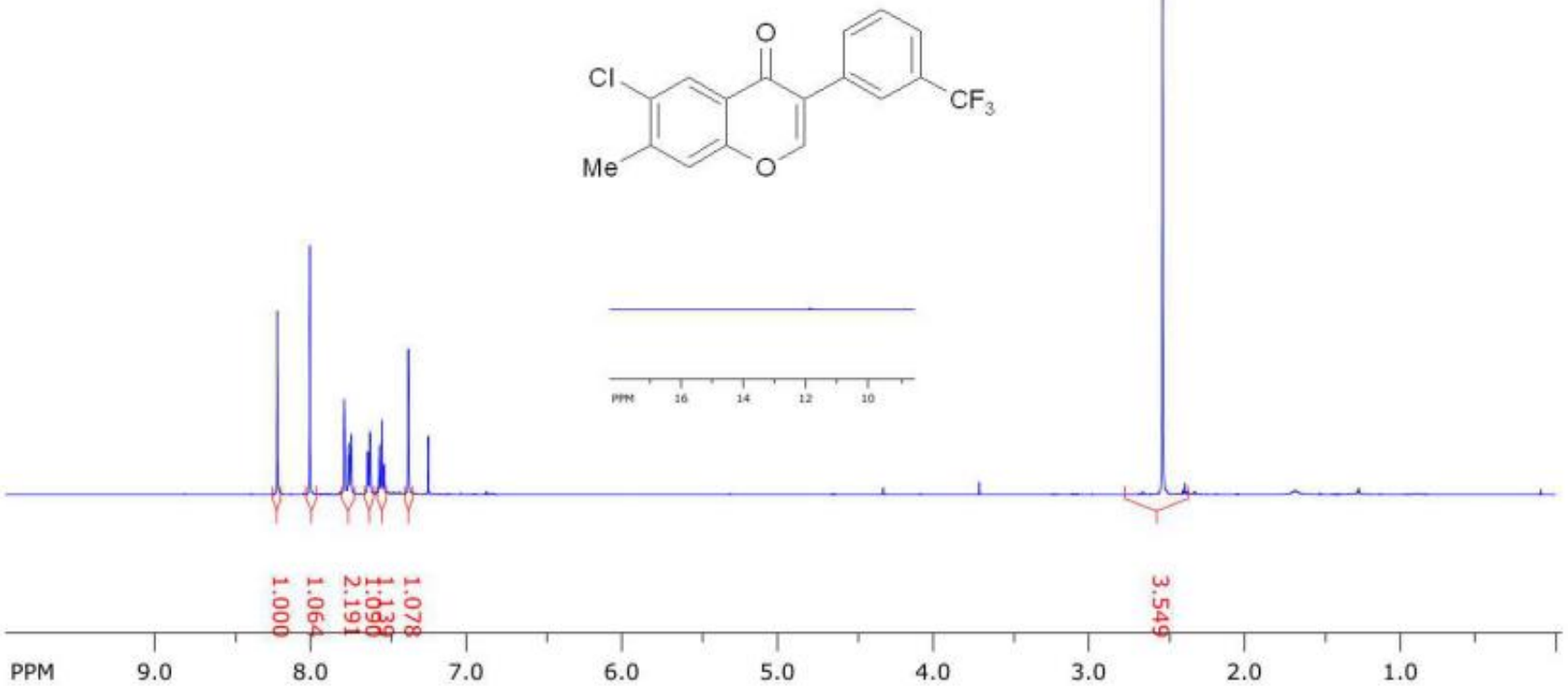

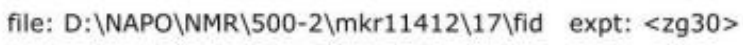
transmitter freq.: $500.133001 \mathrm{MHz}$

time domain size: 65536 points

freq. of 0 ppm: $500.130023 \mathrm{MHz}$

processed size: 65536 complex points

width: $12335.53 \mathrm{~Hz}=24.6645 \mathrm{ppm}=0.188225 \mathrm{~Hz} / \mathrm{pt}$

LB: 0.300 GF: 0.0000

number of scans: 24 


\section{Compound 5v (NMR in $\left.\mathrm{CDCl}_{3}\right)$}

SpinWorks 4: ISM $10813 C$
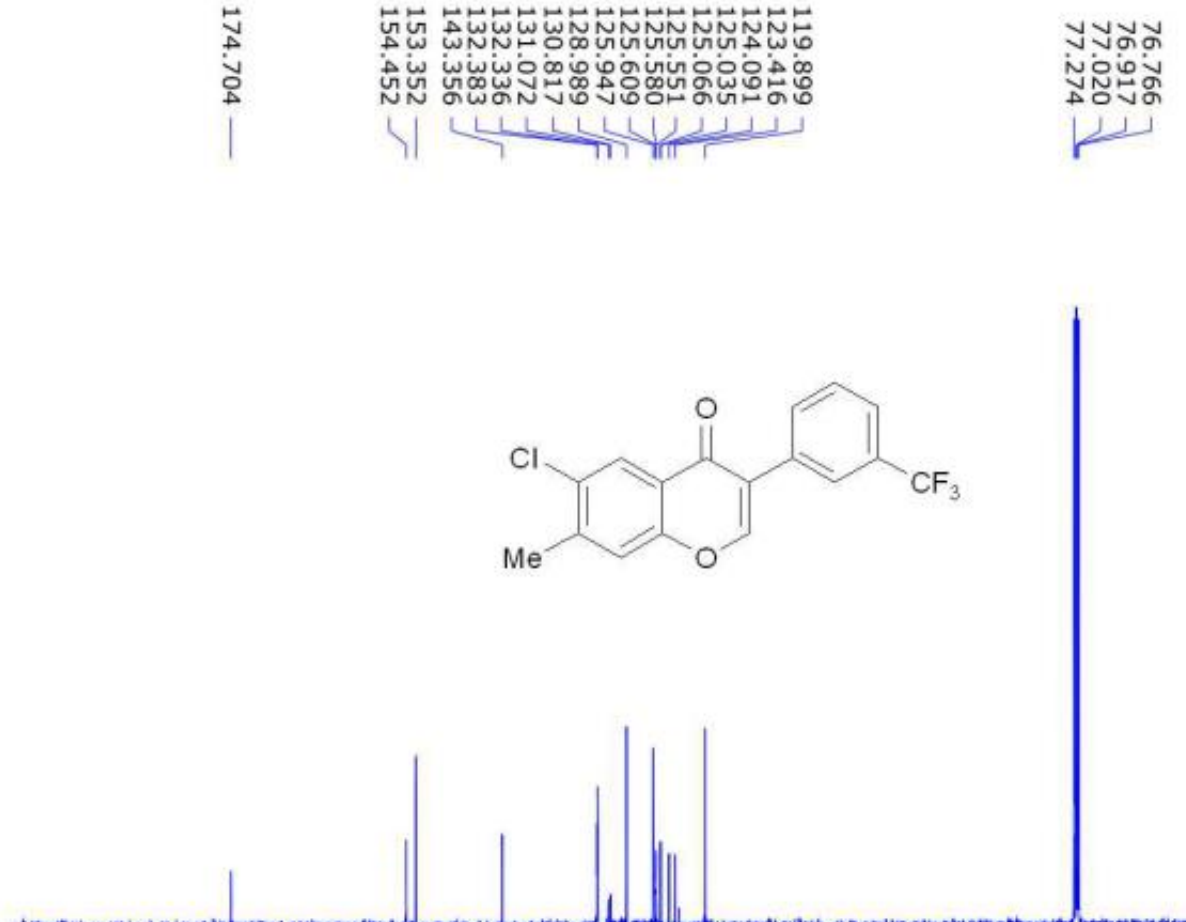
PPM

freq. of $0 \mathrm{ppm}$ : $125.757793 \mathrm{MHz}$

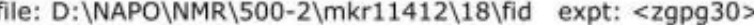
transmitter freq.: $125.772879 \mathrm{MHz}$

processed size: 32768 complex points

time domain size: 65536 points

LB: 2.000 GF: 0.0000

width: $36057.69 \mathrm{~Hz}=286.6889 \mathrm{ppm}=0.550197 \mathrm{~Hz} / \mathrm{pt}$

$\mathrm{Hz} / \mathrm{cm}: 1000.362 \mathrm{ppm} / \mathrm{cm}: 7.95372$ number of scans: 256 


\section{Compound 5w (NMR in $\mathrm{CDCl}_{3}$ )}

SpinWorks 4: ISM 60
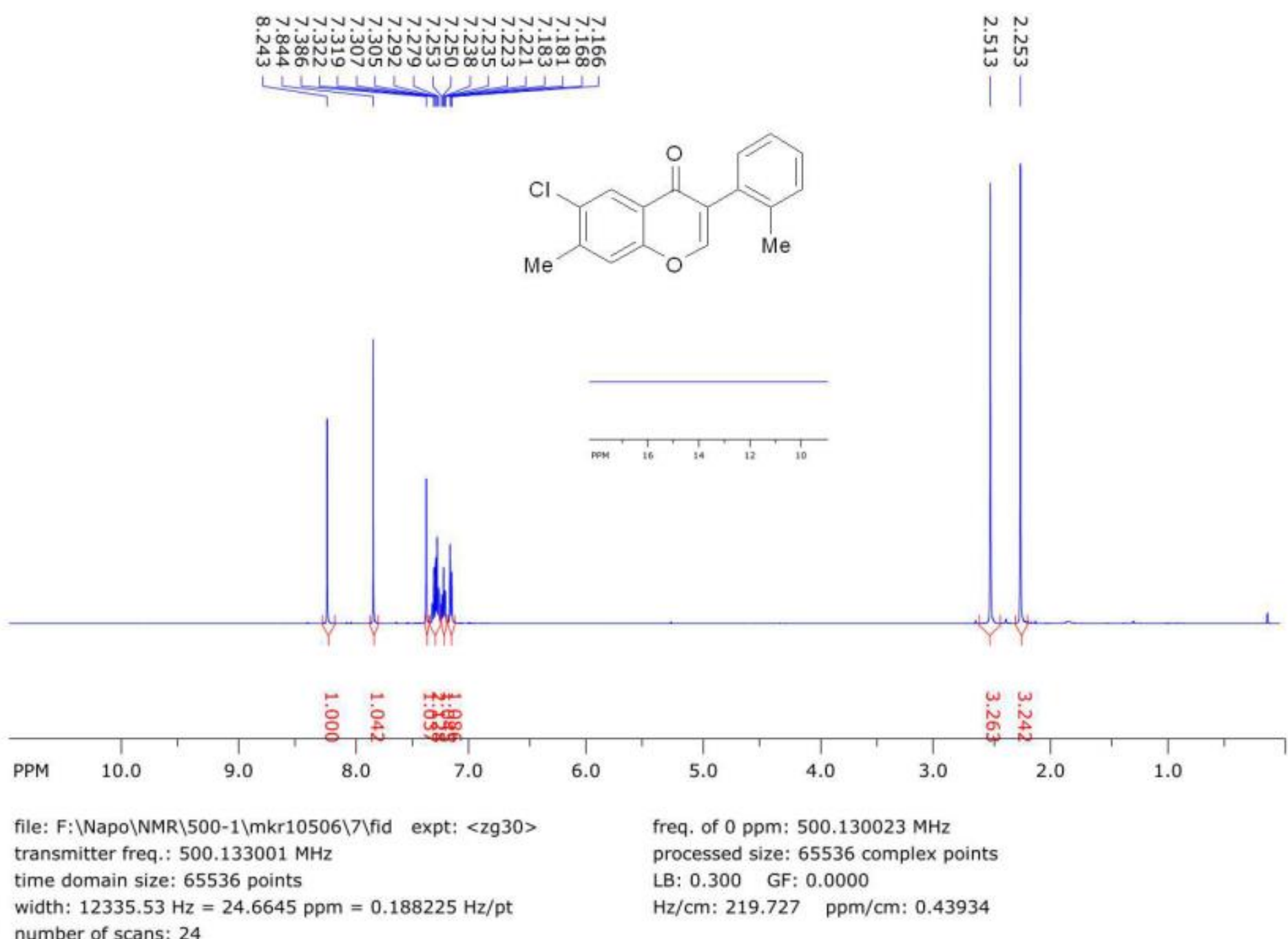


\section{Compound 5w (NMR in $\left.\mathrm{CDCl}_{3}\right)$}

SpinWorks 4: ISM 60
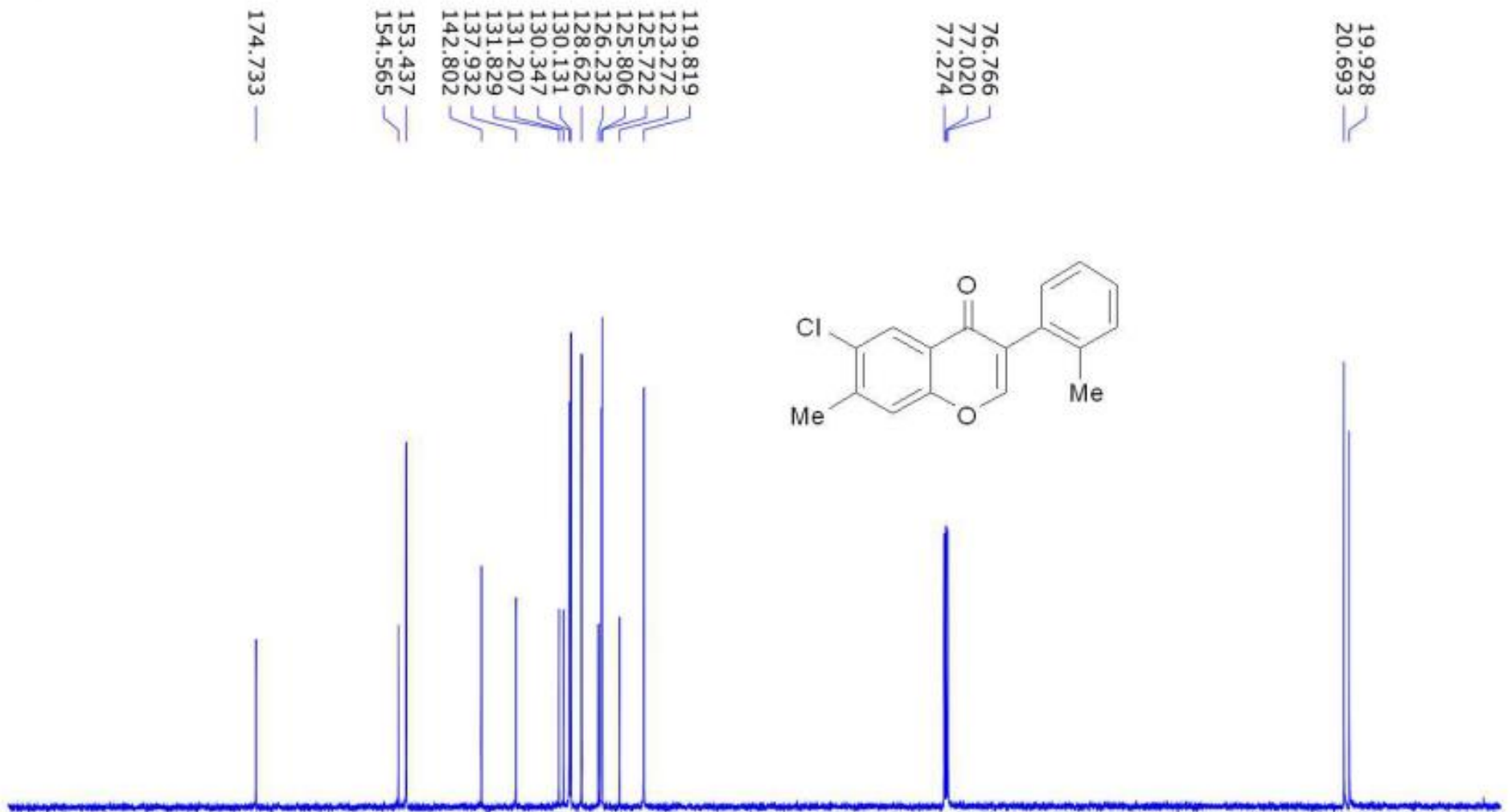

PPM

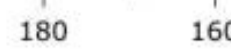

160

140

120

100

80

60

file: F: \Napo\NMR $500-1 \backslash m k r 10506 \backslash 8 \backslash$ fid expt: <zgpg30> transmitter freq.: $125.772879 \mathrm{MHz}$

time domain size: 65536 points

freq. of 0 ppm: $125.757809 \mathrm{MHz}$

width: $36057.69 \mathrm{~Hz}=286.6889 \mathrm{ppm}=0.550197 \mathrm{~Hz} / \mathrm{pt}$ processed size: 32768 complex points

LB: 2.000 GF: 0.0000

number of scans: 512

$\mathrm{Hz} / \mathrm{cm}: 1062.414 \mathrm{ppm} / \mathrm{cm}: 8.44708$ 


\section{Compound 5x (NMR in $\left.\mathrm{CDCl}_{3}\right)$}

SpinWorks 4: ISM 73
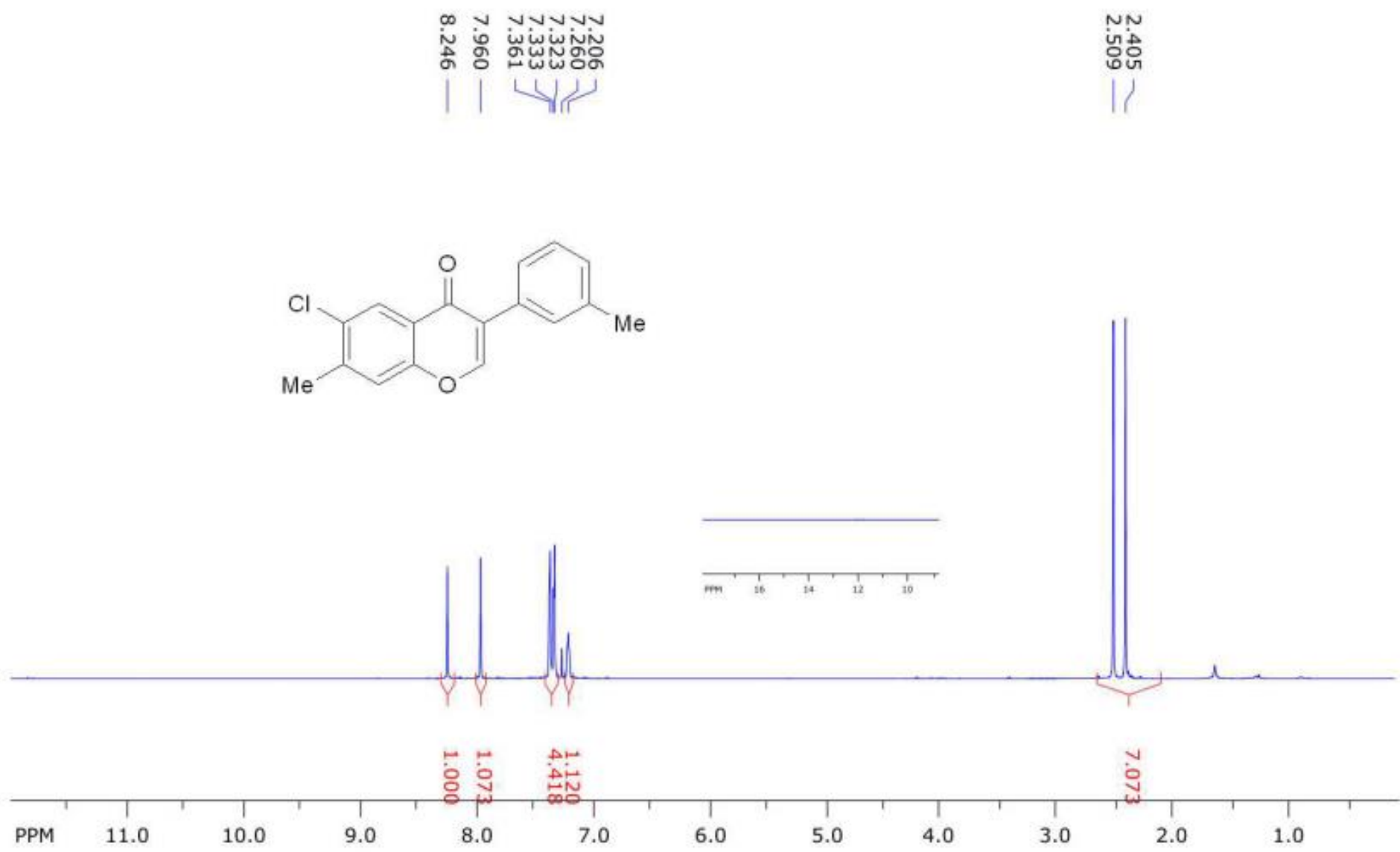

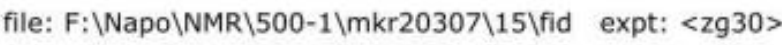
transmitter freq.: $500.133001 \mathrm{MHz}$

time domain size: 65536 points

freq. of $0 \mathrm{ppm}: 500.130023 \mathrm{MHz}$

processed size: 65536 complex points

width: $12335.53 \mathrm{~Hz}=24.6645 \mathrm{ppm}=0.188225 \mathrm{~Hz} / \mathrm{pt}$

LB: 0.300 GF: 0.0000

number of scans: 24

$\mathrm{Hz} / \mathrm{cm}: 238.450 \mathrm{ppm} / \mathrm{cm}: 0.47677$ 


\section{Compound 5x (NMR in $\left.\mathrm{CDCl}_{3}\right)$}

SpinWorks 4: ISM 73
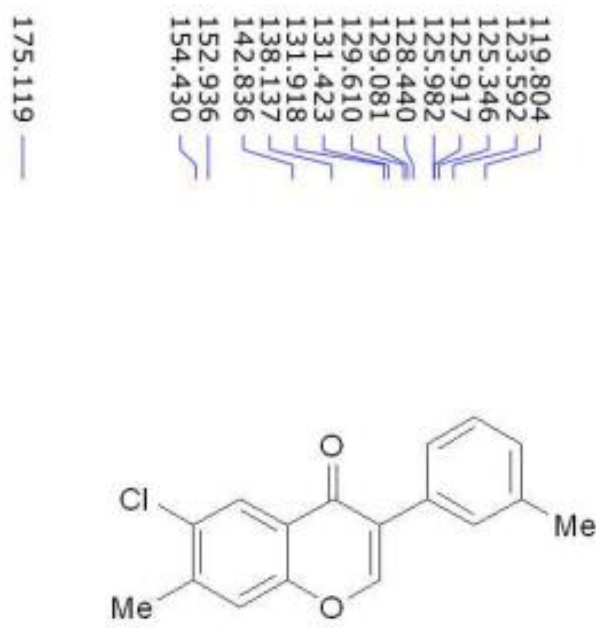

Me 


\section{Compound 5y (NMR in $\mathrm{CDCl}_{3}$ )}

SpinWorks 4: ISM $2151 \mathrm{H}$
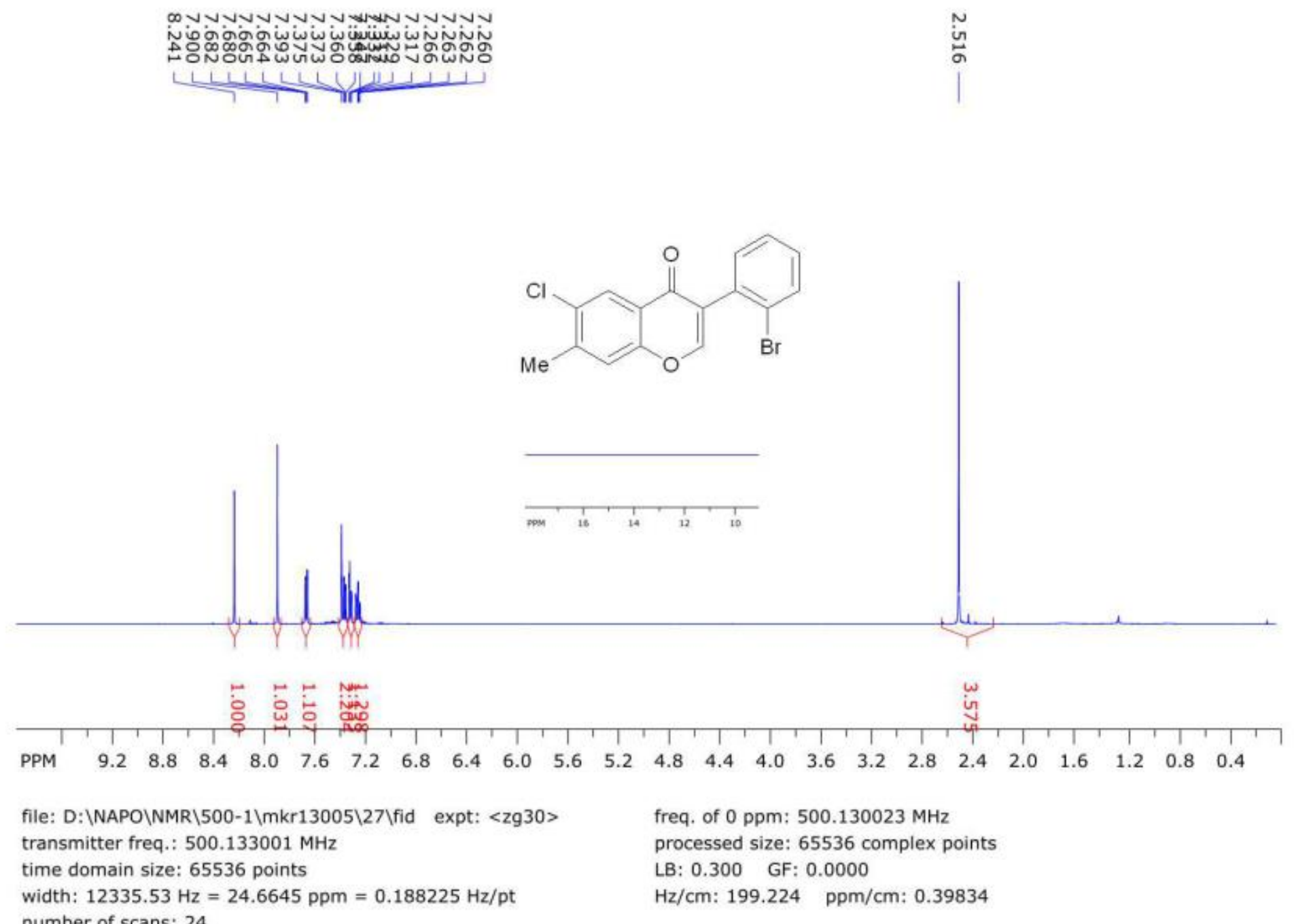


\section{Compound 5y (NMR in $\left.\mathrm{CDCl}_{3}\right)$}

SpinWorks 4: ISM $21513 \mathrm{C}$
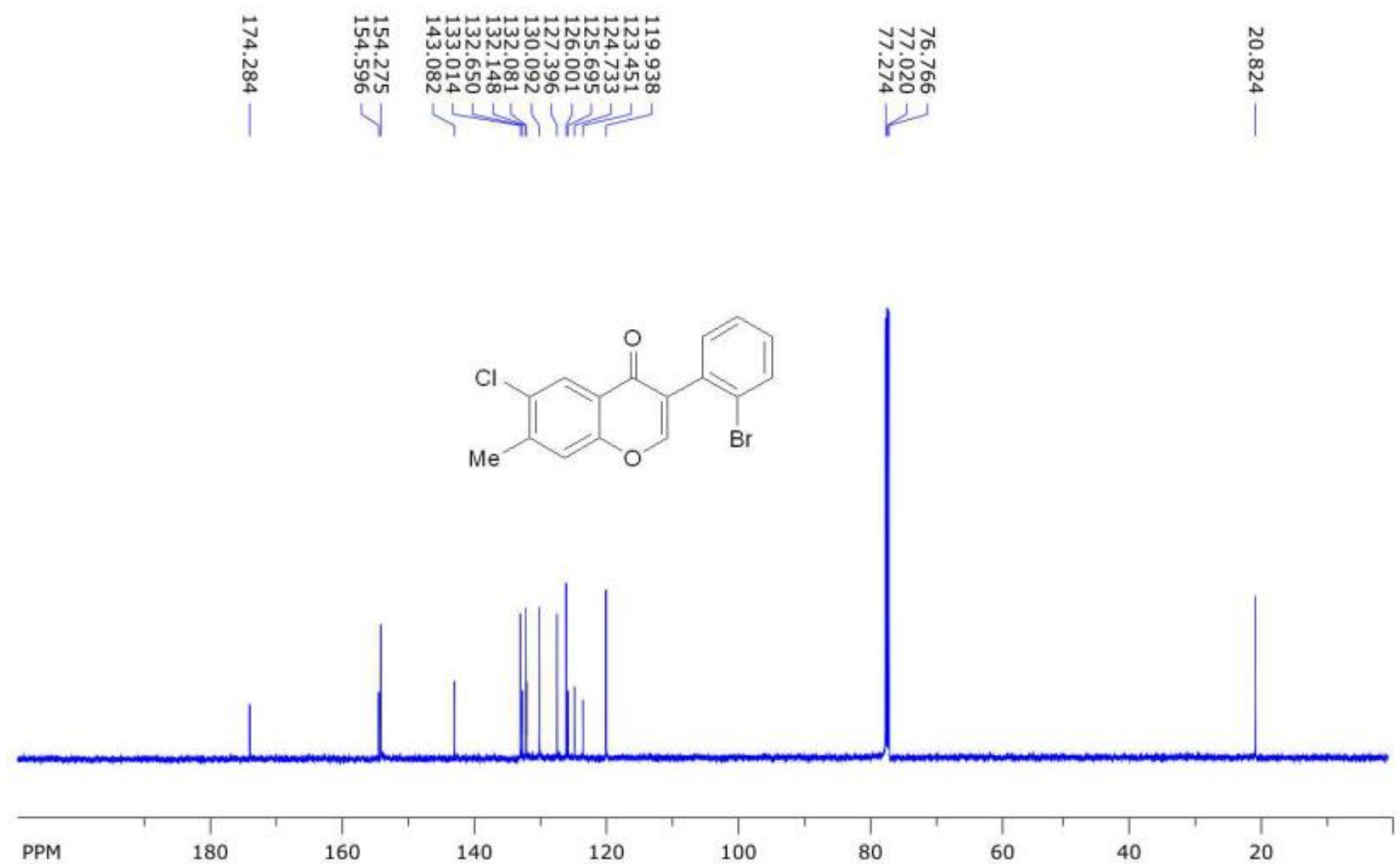

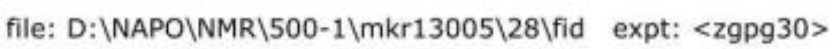
transmitter freq.: $125.772879 \mathrm{MHz}$

freq. of $0 \mathrm{ppm}: 125.757797 \mathrm{MHz}$

time domain size: 65536 points

processed size: 32768 complex points

width: $36057.69 \mathrm{~Hz}=286.6889 \mathrm{ppm}=0.550197 \mathrm{~Hz} / \mathrm{pt}$

LB: 2.000 GF: 0.0000

number of scans: 512 


\section{Compound 5z (NMR in $\left.\mathrm{CDCl}_{3}\right)$}

SpinWorks 4: ISM 27

$\infty \infty \infty \infty \infty \vee V V V V V V V V V$

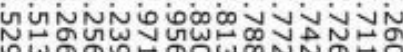

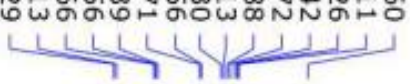

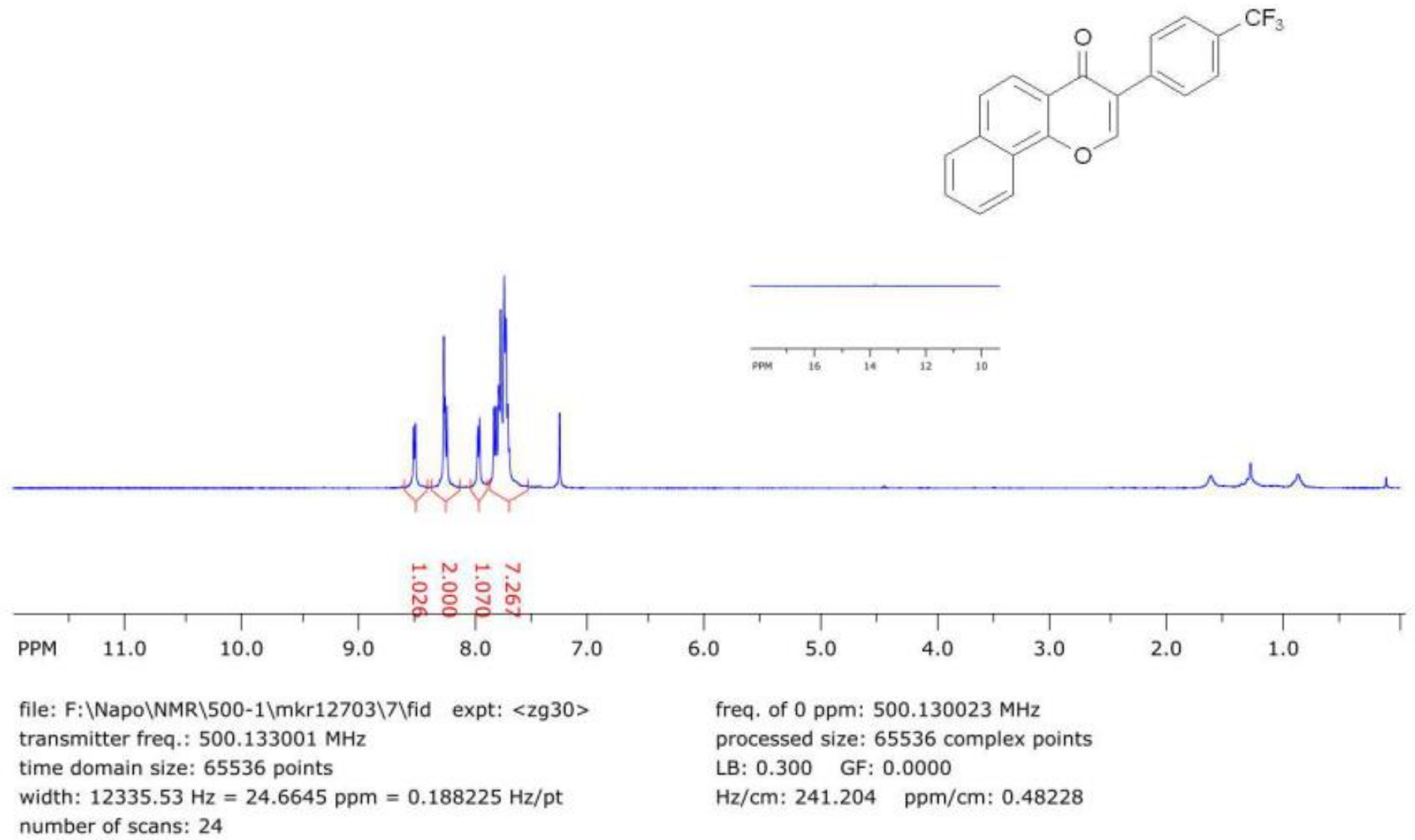




\section{Compound 5aa (NMR in $\mathrm{CDCl}_{3}$ )}

SpinWorks 4: ISM 129 1H

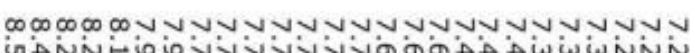
जڤNN

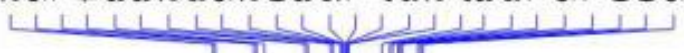
1 II Thi III

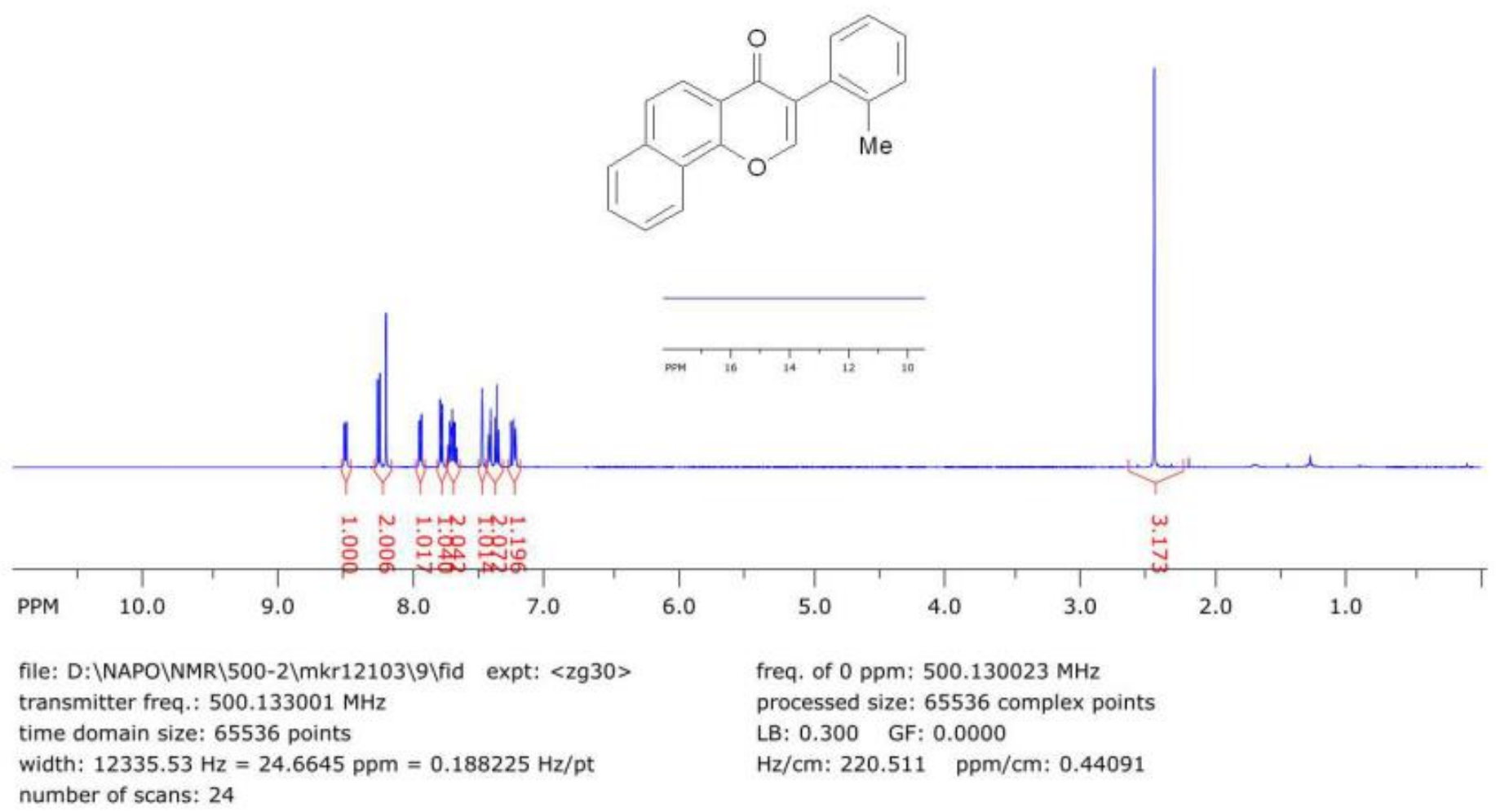




\section{Compound 5aa (NMR in $\left.\mathrm{CDCl}_{3}\right)$}

SpinWorks 4: ISM 129 13C
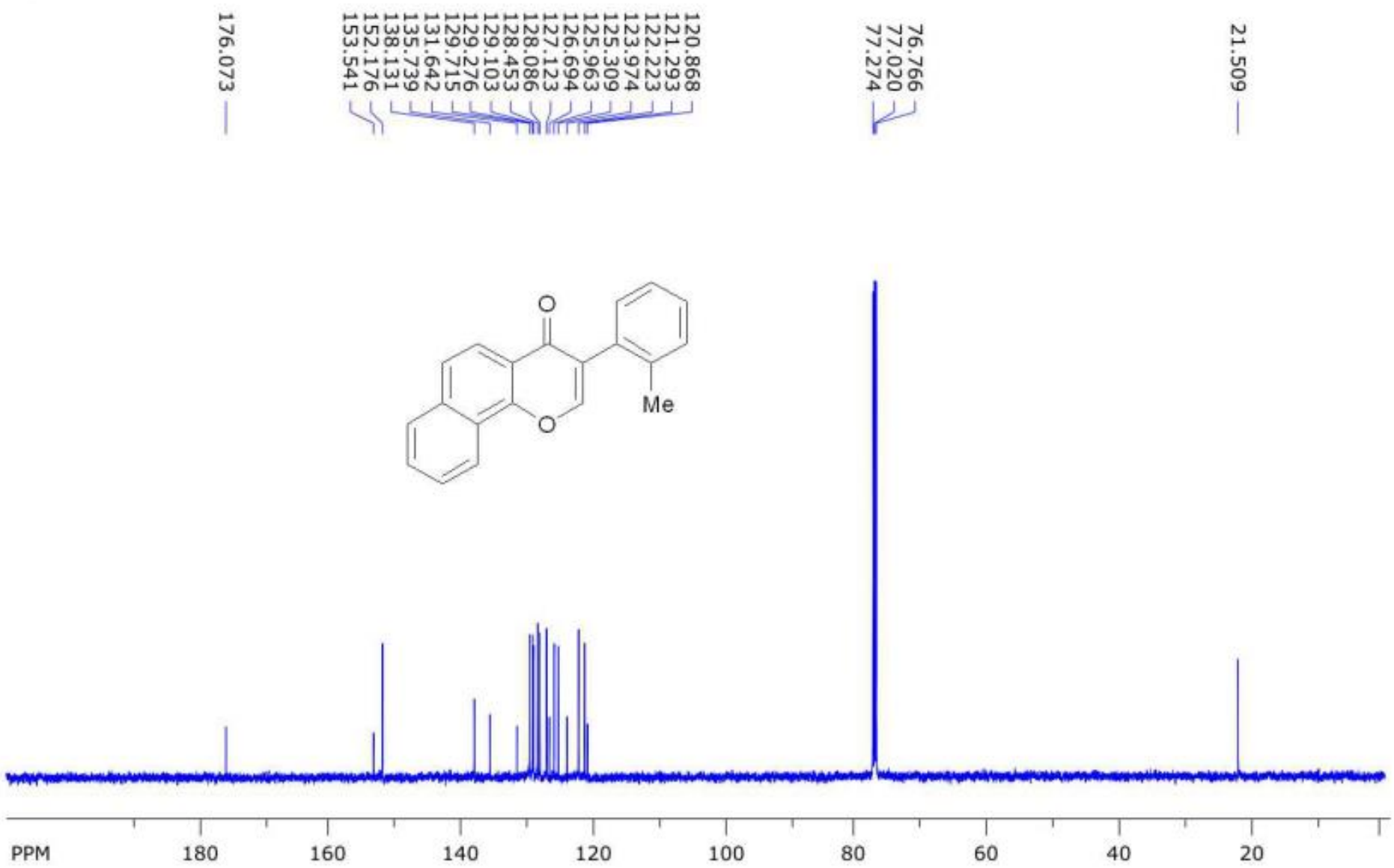

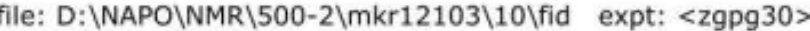
transmitter freq.: $125.772879 \mathrm{MHz}$

time domain size: 65536 points

freq. of 0 ppm: $125.757796 \mathrm{MHz}$

processed size: 32768 complex points

LB: 2.000 GF: 0.0000

width: $36057.69 \mathrm{~Hz}=286.6889 \mathrm{ppm}=0.550197 \mathrm{~Hz} / \mathrm{pt}$

$\mathrm{Hz} / \mathrm{cm}: 1058.761$

$\mathrm{ppm} / \mathrm{cm}: 8.41804$

number of scans: 512 


\section{Compound 5ab (NMR in $\left.\mathrm{CDCl}_{3}\right)$}

SpinWorks 4: ISM 97-2 1H

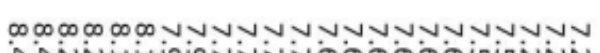
ค A N

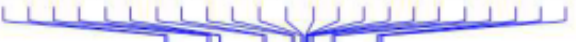

11 1 ता
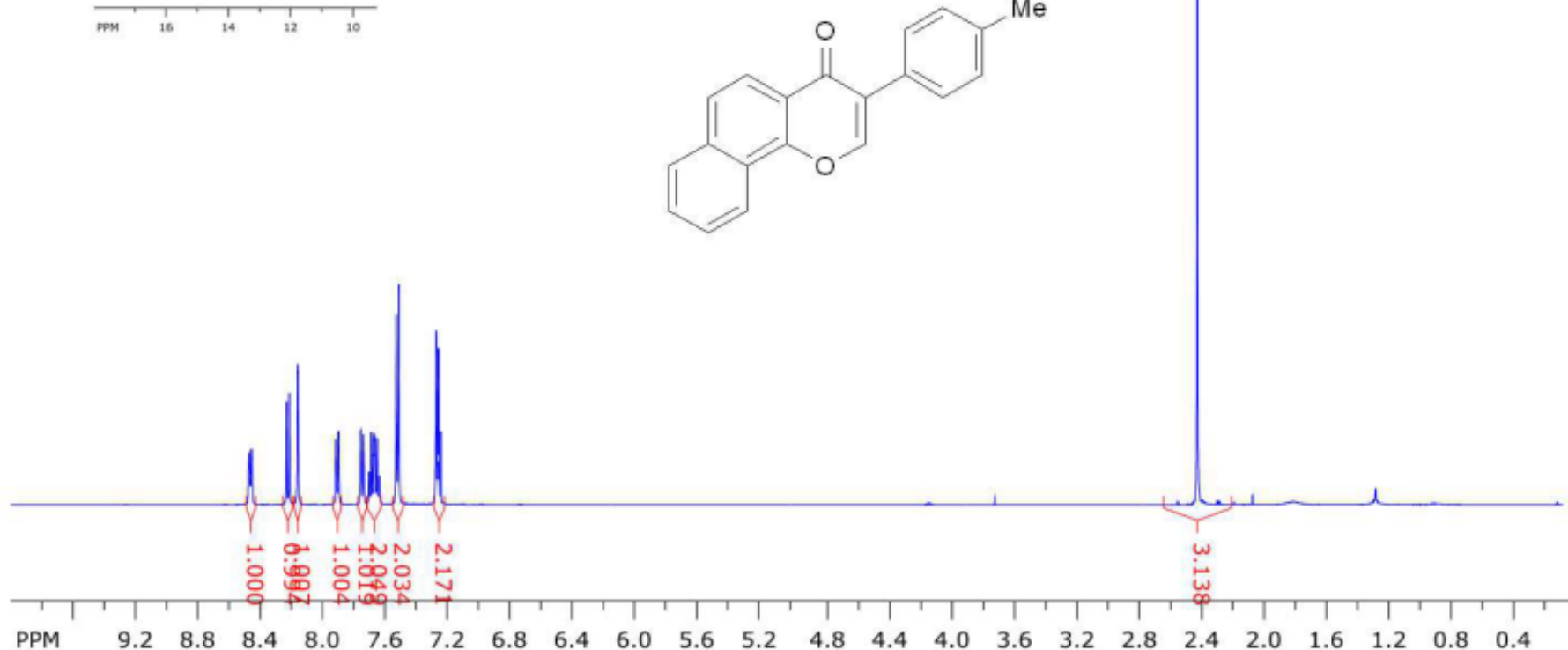

PPM

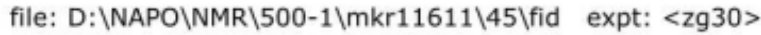

transmitter freq.: $500.133001 \mathrm{MHz}$

time domain size: 65536 points

freq. of $0 \mathrm{ppm}$ : $500.130023 \mathrm{MHz}$

$\stackrel{N}{\stackrel{n}{0}}$

width: $12335.53 \mathrm{~Hz}=24.6645 \mathrm{ppm}=0.188225 \mathrm{~Hz} / \mathrm{pt}$

processed size: 65536 complex points

LB: 0.300 GF: 0.0000

number of scans: 16

$\mathrm{Hz} / \mathrm{cm}: 199.224 \mathrm{ppm} / \mathrm{cm}: 0.39834$ 


\section{Compound 5ab (NMR in $\left.\mathrm{CDCl}_{3}\right)$}

SpinWorks 4: ISM 97-2 13C
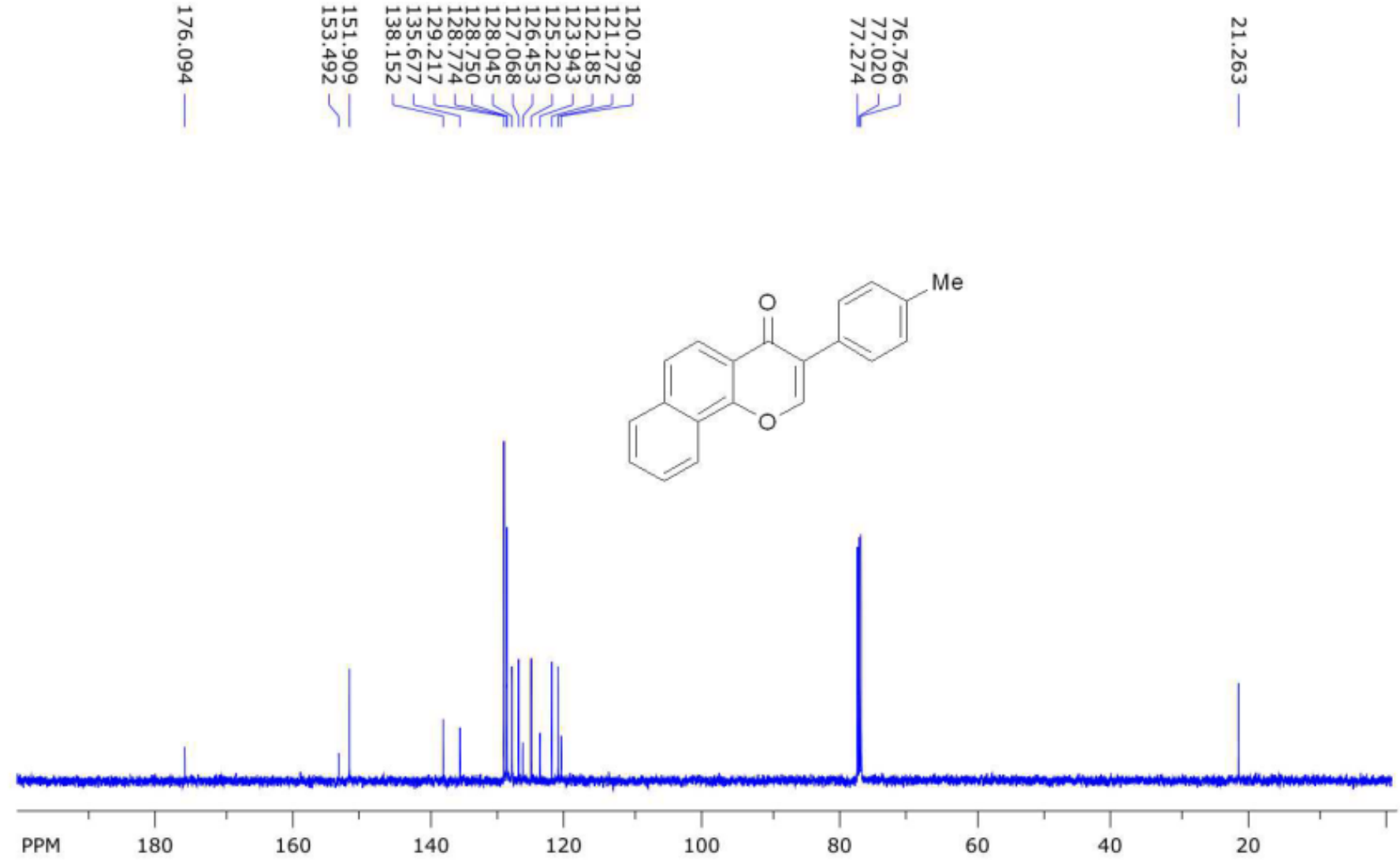

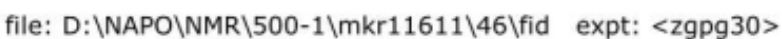
transmitter freq.: $125.772879 \mathrm{MHz}$

freq. of $0 \mathrm{ppm}$ : $125.757798 \mathrm{MHz}$

time domain size: 65536 points

processed size: 32768 complex points

width: $36057.69 \mathrm{~Hz}=286.6889 \mathrm{ppm}=0.550197 \mathrm{~Hz} / \mathrm{pt}$

LB: 2.000 GF: 0.0000

number of scans: 128

$\mathrm{Hz} / \mathrm{cm}: 1016.316 \mathrm{ppm} / \mathrm{cm}: 8.08057$ 


\section{Compound 5ac (NMR in $\mathrm{CDCl}_{3}$ )}

SpinWorks 4: ISM 132-1 $1 \mathrm{H}$

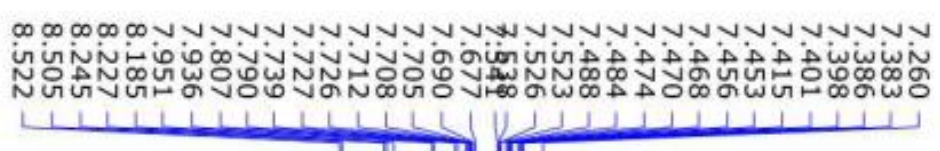

प्राता in
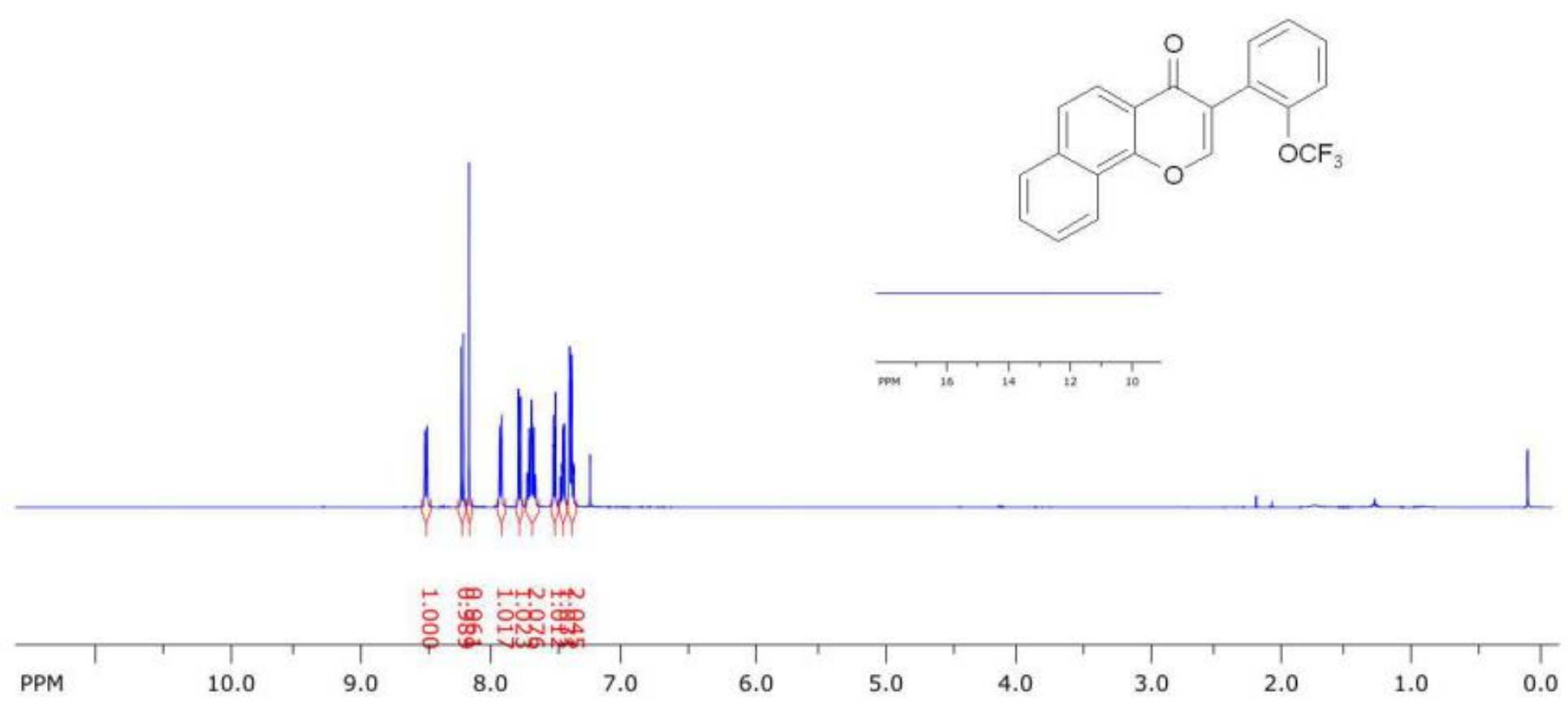

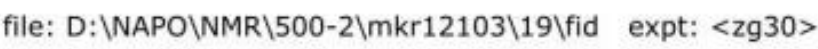
transmitter freq.: $500.133001 \mathrm{MHz}$

time domain size: 65536 points

freq. of $0 \mathrm{ppm}$ : $500.130023 \mathrm{MHz}$

processed size: 65536 complex points

width: $12335.53 \mathrm{~Hz}=24.6645 \mathrm{ppm}=0.188225 \mathrm{~Hz} / \mathrm{pt}$

LB: 0.300 GF: 0.0000

number of scans: 24 


\section{Compound 5ac (NMR in $\left.\mathrm{CDCl}_{3}\right)$}

SpinWorks 4: ISM 132-1 13C
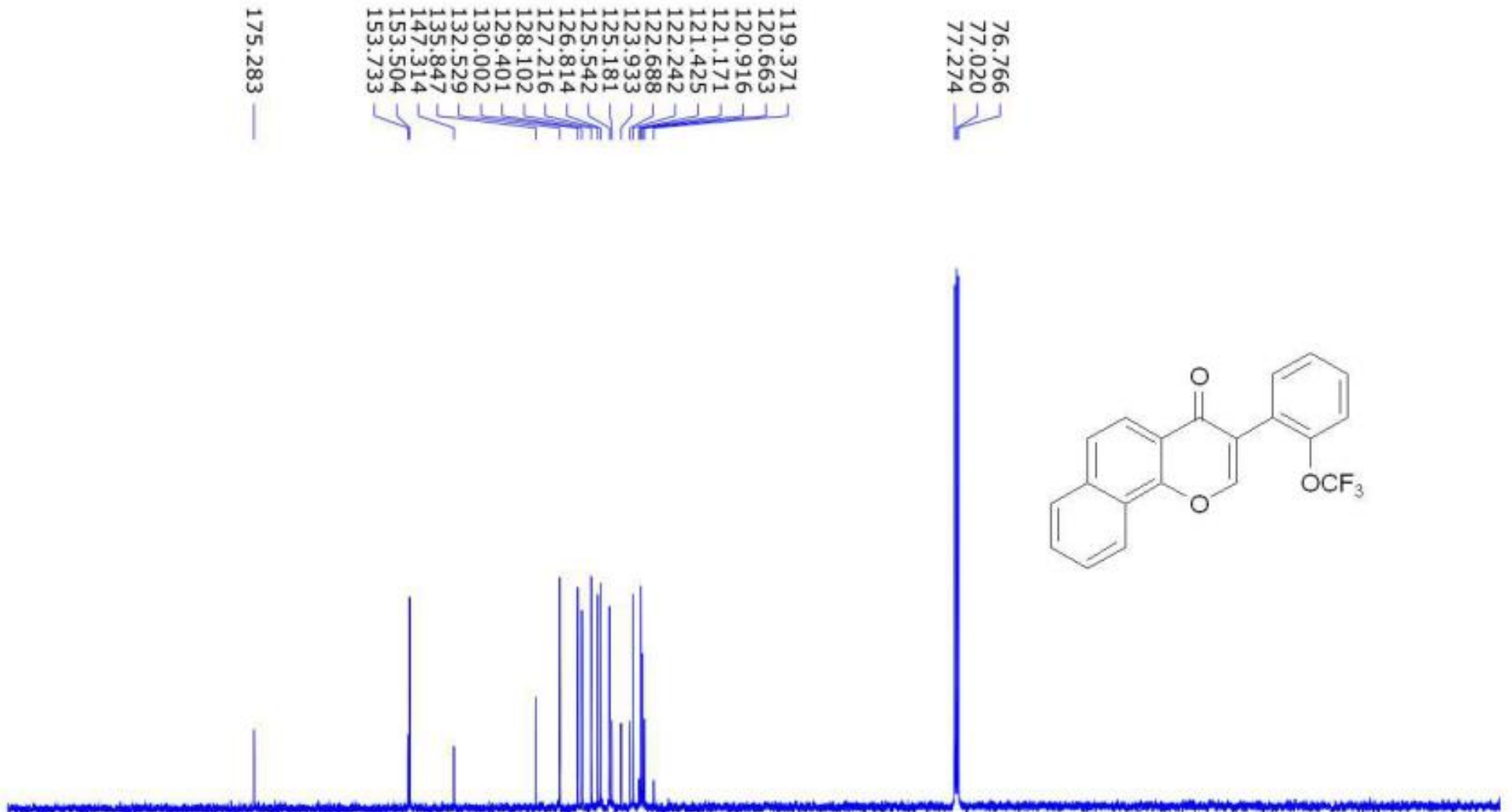

PPM

file: D:\NAPO\NMR $500-2 \backslash \mathrm{mkr} 12103 \backslash 20 \backslash$ fid expt: <zgpg30> transmitter freq.: $125.772879 \mathrm{MHz}$

time domain size: 65536 points

width: $36057.69 \mathrm{~Hz}=286.6889 \mathrm{ppm}=0.550197 \mathrm{~Hz} / \mathrm{pt}$ numher of erane. 51 ? freq. of 0 ppm: $125.757796 \mathrm{MHz}$ processed size: 32768 complex points LB: 2.000 GF: 0.0000

$\mathrm{Hz} / \mathrm{cm}: 1049.821 \mathrm{ppm} / \mathrm{cm}: 8.34696$ 


\section{Compound 5ad (NMR in $\left.\mathrm{CDCl}_{3}\right)$}

SpinWorks 4: ISM 117-2 1H

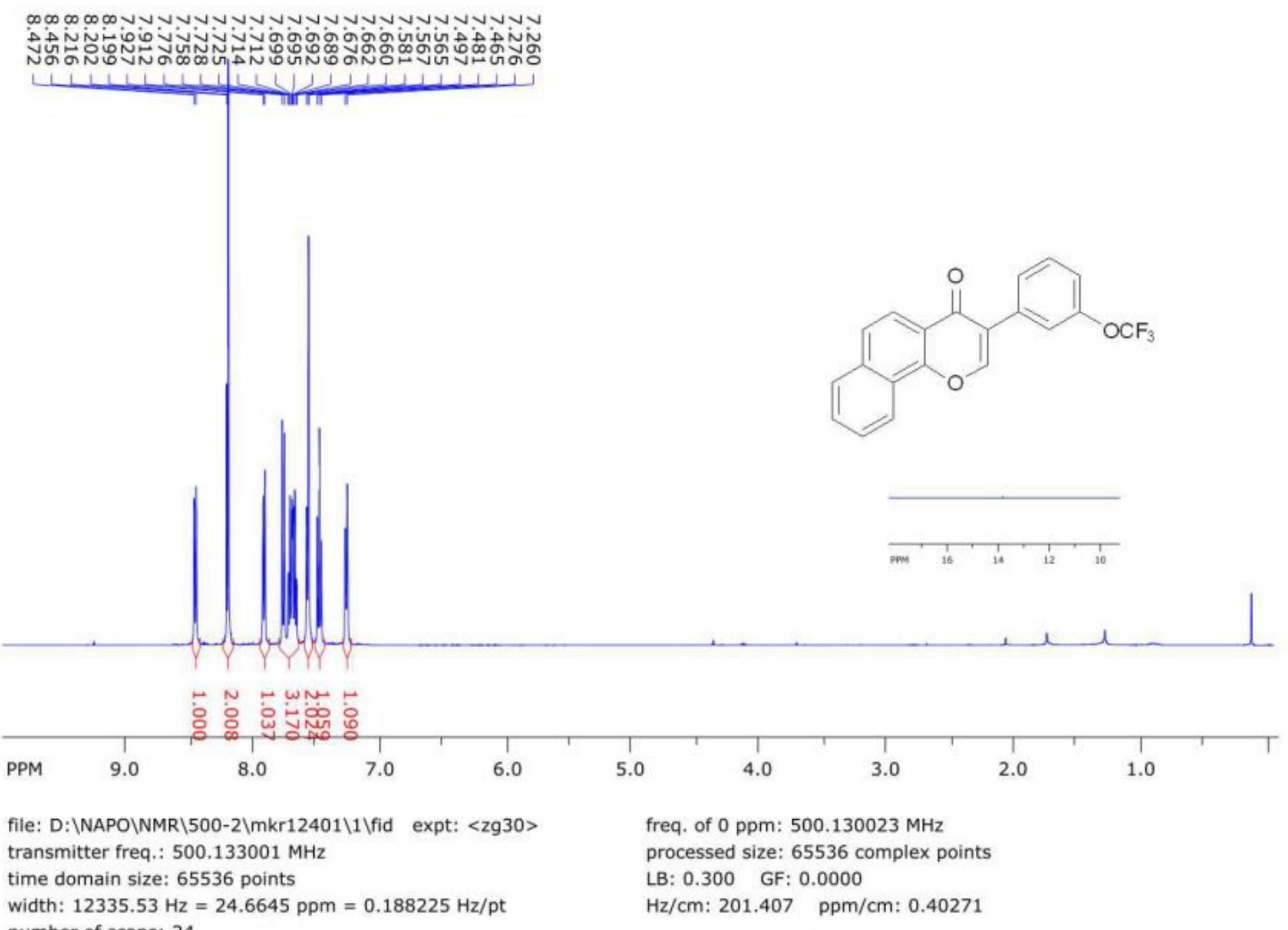




\section{Compound 5ad (NMR in $\left.\mathrm{CDCl}_{3}\right)$}

SpinWorks 4: ISM 117-2 13C
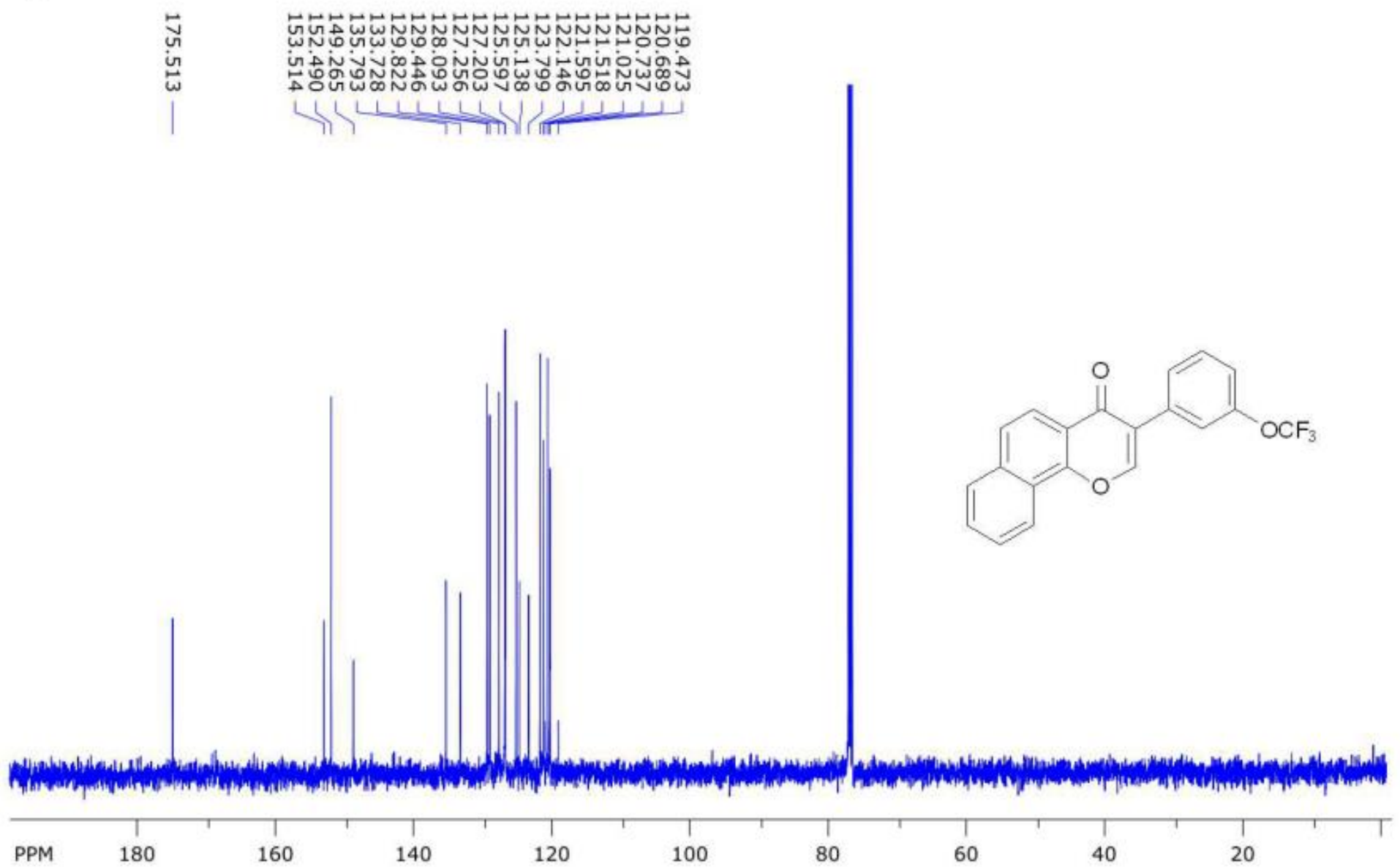

PPM

180

expt: <zgpg30>

file: $D: \backslash N A P O \backslash N M R \backslash 500-2 \backslash m \mathrm{kr} 12401 \backslash$
transmitter freq.: $125.772879 \mathrm{MHz}$

time domain size: 65536 points

freq. of 0 ppm: $125.757797 \mathrm{MHz}$

width: $36057.69 \mathrm{~Hz}=286.6889 \mathrm{ppm}=0.550197 \mathrm{~Hz} / \mathrm{pt}$

processed size: 32768 complex points

number of scans: 128 


\section{Compound 5ae (NMR in $\mathrm{CDCl}_{3}$ )}

SpinWorks 4: ISM 16
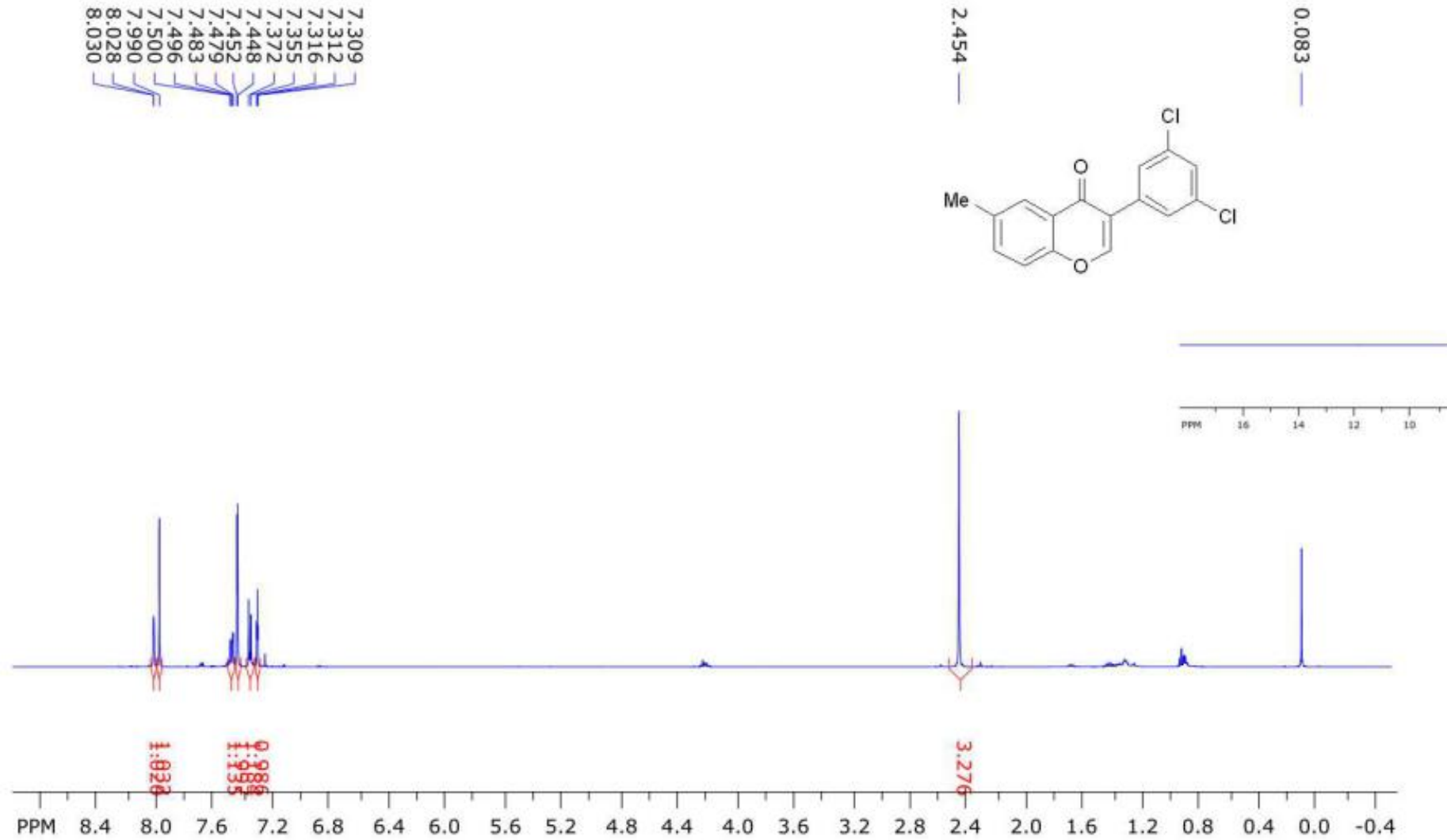

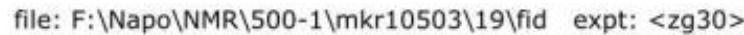
transmitter freq.: $500.133001 \mathrm{MHz}$

time domain size: 65536 points

freq. of 0 ppm: $500.130024 \mathrm{MHz}$

width: $12335.53 \mathrm{~Hz}=24.6645 \mathrm{ppm}=0.188225 \mathrm{~Hz} / \mathrm{pt} \quad \mathrm{Hz} / \mathrm{cm}: 191.091 \mathrm{ppm} / \mathrm{cm}: 0.38208$

number of scans: 24 


\section{Compound 5ae (NMR in $\mathrm{CDCl}_{3}$ )}

SpinWorks 4: ISM 16

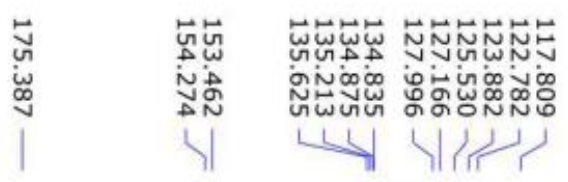

ทีู่

กู่ิ่ปั

H
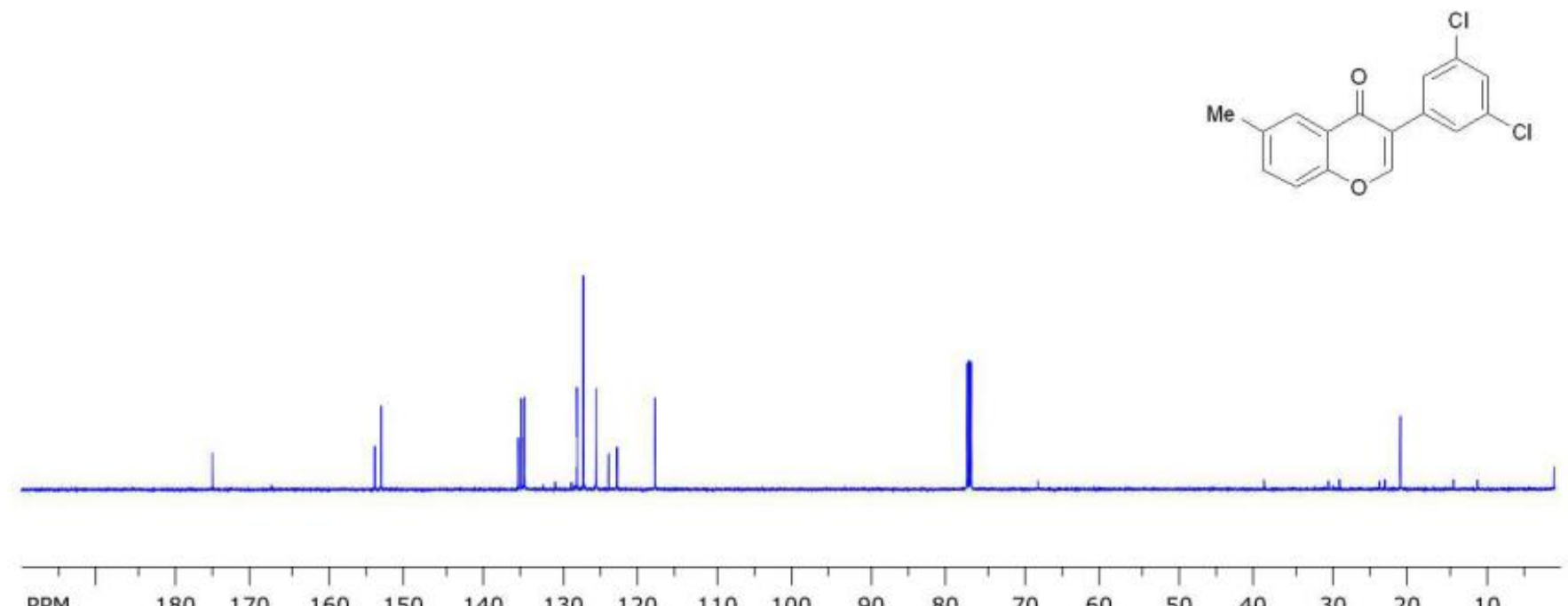

PPM
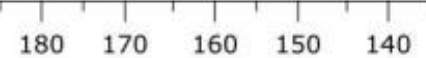

$130 \quad 120$
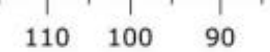

80

60

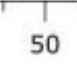

40

3020

10

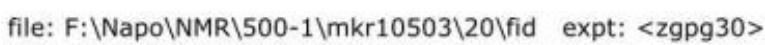
transmitter freq.: $125.772879 \mathrm{MHz}$

time domain size: 65536 points

width: $36057.69 \mathrm{~Hz}=286.6889 \mathrm{ppm}=0.550197 \mathrm{~Hz} / \mathrm{pt}$

number of scans: 512 freq. of $0 \mathrm{ppm}$ : $125.757800 \mathrm{MHz}$

processed size: 32768 complex points

LB: 2.000 GF: 0.0000

$\mathrm{Hz} / \mathrm{cm}$ : $1002.855 \mathrm{ppm} / \mathrm{cm}: 7.97354$ 


\section{Compound 5af (NMR in $\left.\mathrm{CDCl}_{3}\right)$}

SpinWorks 4: ISM 30

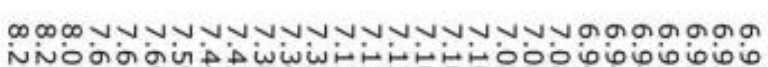
N רา 1 ก<smiles>COc1cccc(-c2coc3ccc(Cl)cc3c2=O)c1</smiles>
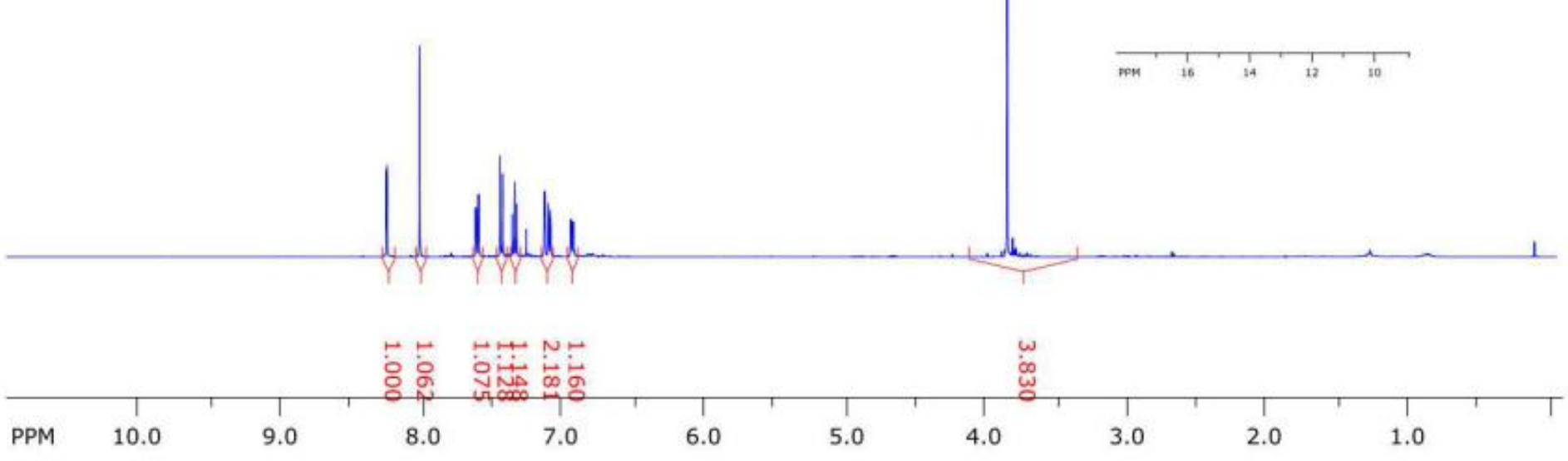

file: $F: \backslash N a p o \backslash N M R \backslash 500-1 \backslash m k r 12703 \backslash 1 \backslash f i d$ expt: $\langle z g 30\rangle$ transmitter freq.: $500.133001 \mathrm{MHz}$

time domain size: 65536 points

freq. of 0 ppm: $500.130024 \mathrm{MHz}$

width: $12335.53 \mathrm{~Hz}=24.6645 \mathrm{ppm}=0.188225 \mathrm{~Hz} / \mathrm{pt}$

processed size: 65536 complex points

LB: 0.300 GF: 0.0000

$\mathrm{Hz} / \mathrm{cm}: 220.828 \mathrm{ppm} / \mathrm{cm}: 0.44154$

number of scans: 24 


\section{Compound 5af (NMR in $\left.\mathrm{CDCl}_{3}\right)$}

SpinWorks 4: ISM 30

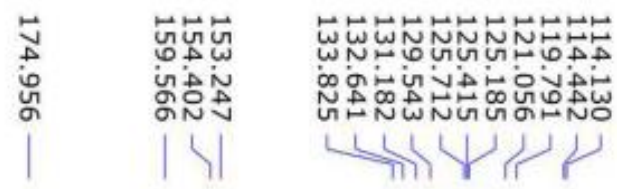

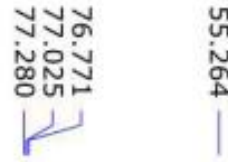
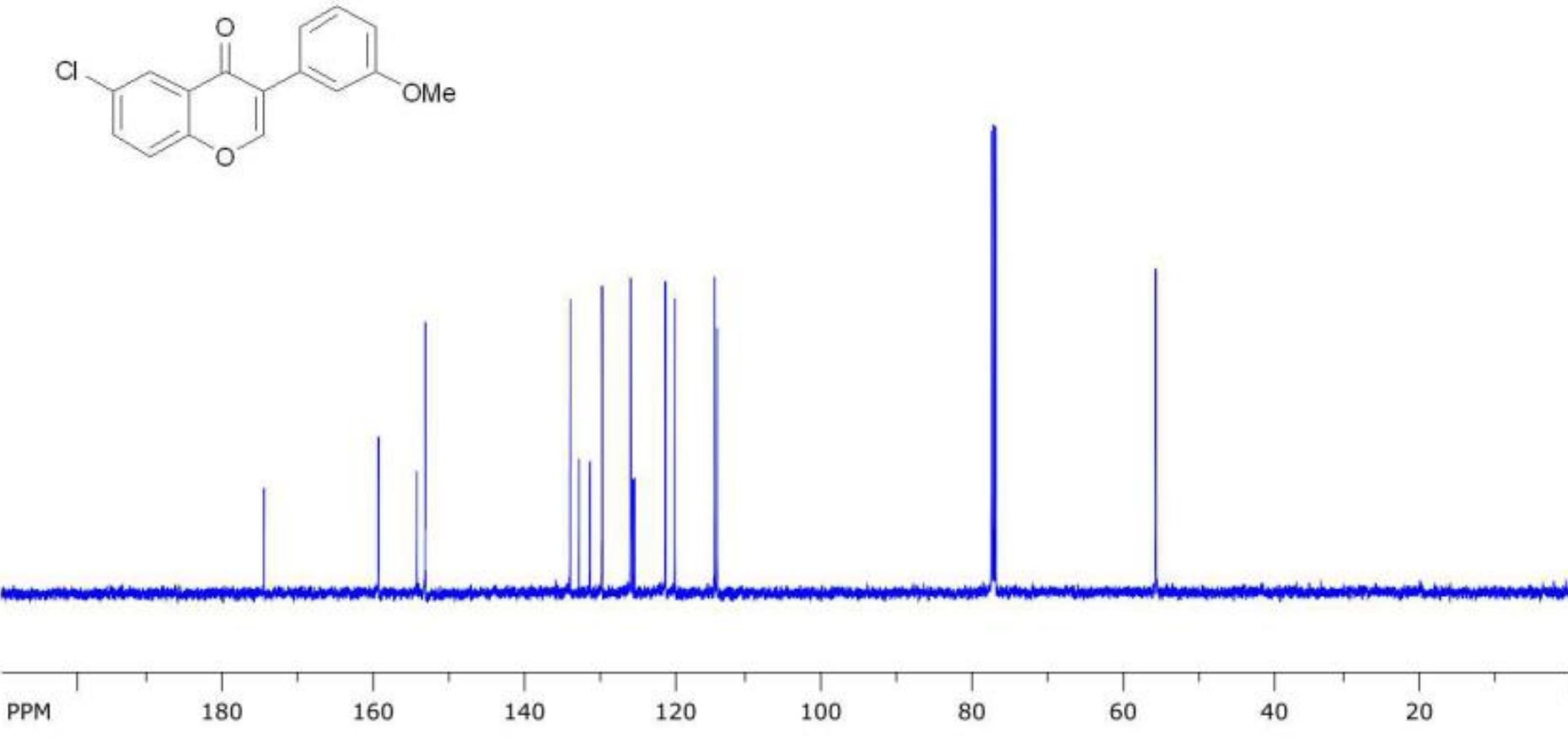

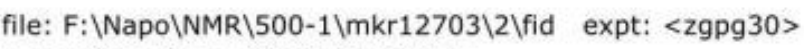
transmitter freq.: $125.772879 \mathrm{MHz}$

time domain size: 65536 points

width: $36057.69 \mathrm{~Hz}=286.6889 \mathrm{ppm}=0.550197 \mathrm{~Hz} / \mathrm{pt}$

number of scans: 256 


\section{Compound 5ag (NMR in $\left.\mathrm{CDCl}_{3}\right)$}

SpinWorks 4: ISM 116 1H
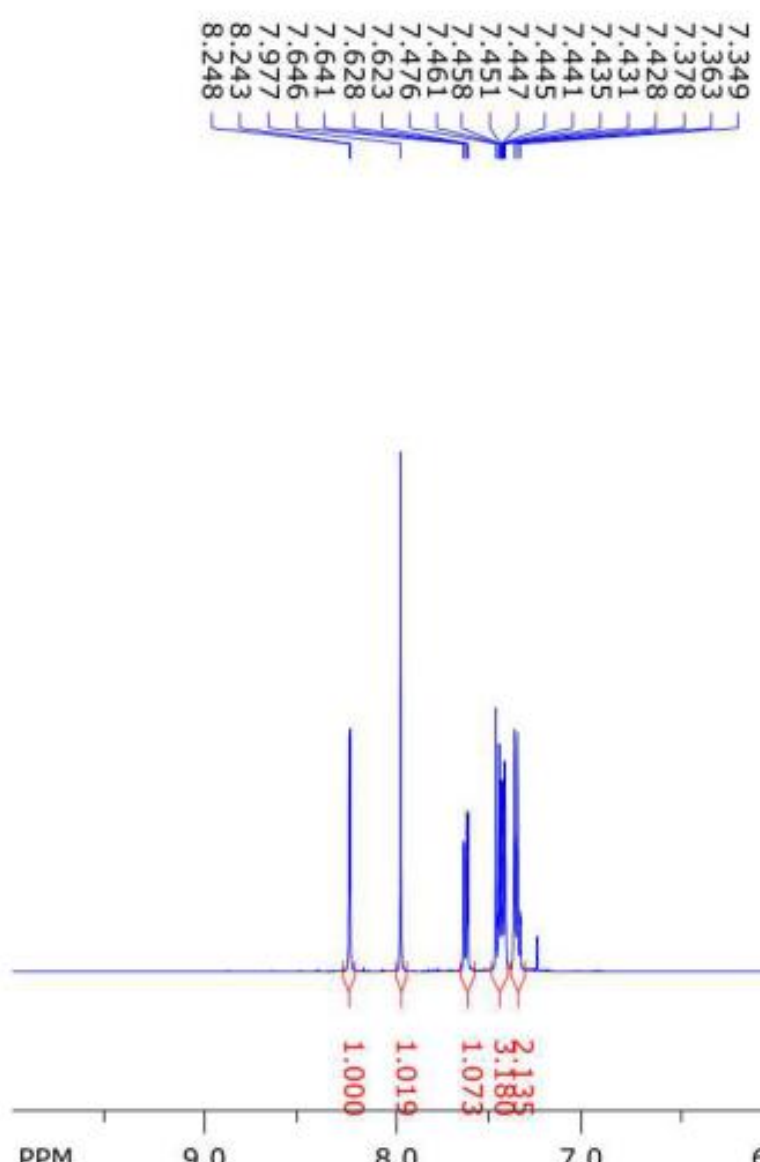

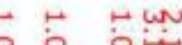

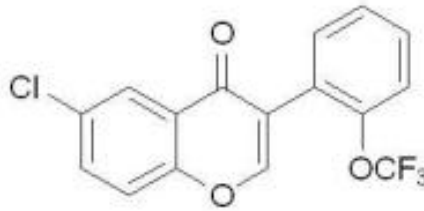

9.0

8.0

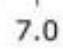

6.0

5.0

4.0

3.0

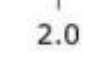

freq. of $0 \mathrm{ppm}$ : $500.130023 \mathrm{MHz}$

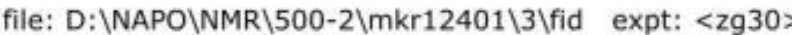
transmitter freq.: $500.133001 \mathrm{MHz}$

time domain size: 65536 points

processed size: 65536 complex points

width: $12335.53 \mathrm{~Hz}=24.6645 \mathrm{ppm}=0.188225 \mathrm{~Hz} / \mathrm{pt}$

LB: 0.300 GF: 0.0000

number of scans: 24 


\section{Compound 5ag (NMR in $\left.\mathrm{CDCl}_{3}\right)$}

SpinWorks 4: ISM $11613 \mathrm{C}$


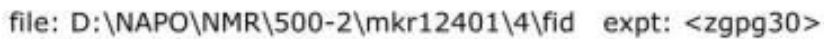

freq. of 0 ppm: $125.757797 \mathrm{MHz}$

transmitter freq.: $125.772879 \mathrm{MHz}$

processed size: 32768 complex points

time domain size: 65536 points

LB: 2.000 GF: 0.0000

width: $36057.69 \mathrm{~Hz}=286.6889 \mathrm{ppm}=0.550197 \mathrm{~Hz} / \mathrm{pt}$

$\mathrm{Hz} / \mathrm{cm}: 1006.744$ ppm/cm: 8.00446

number of scans: 96 


\section{Compound 5ah (NMR in $\left.\mathrm{CDCl}_{3}\right)$}

SpinWorks 4: ISM $101 \mathrm{1H}$
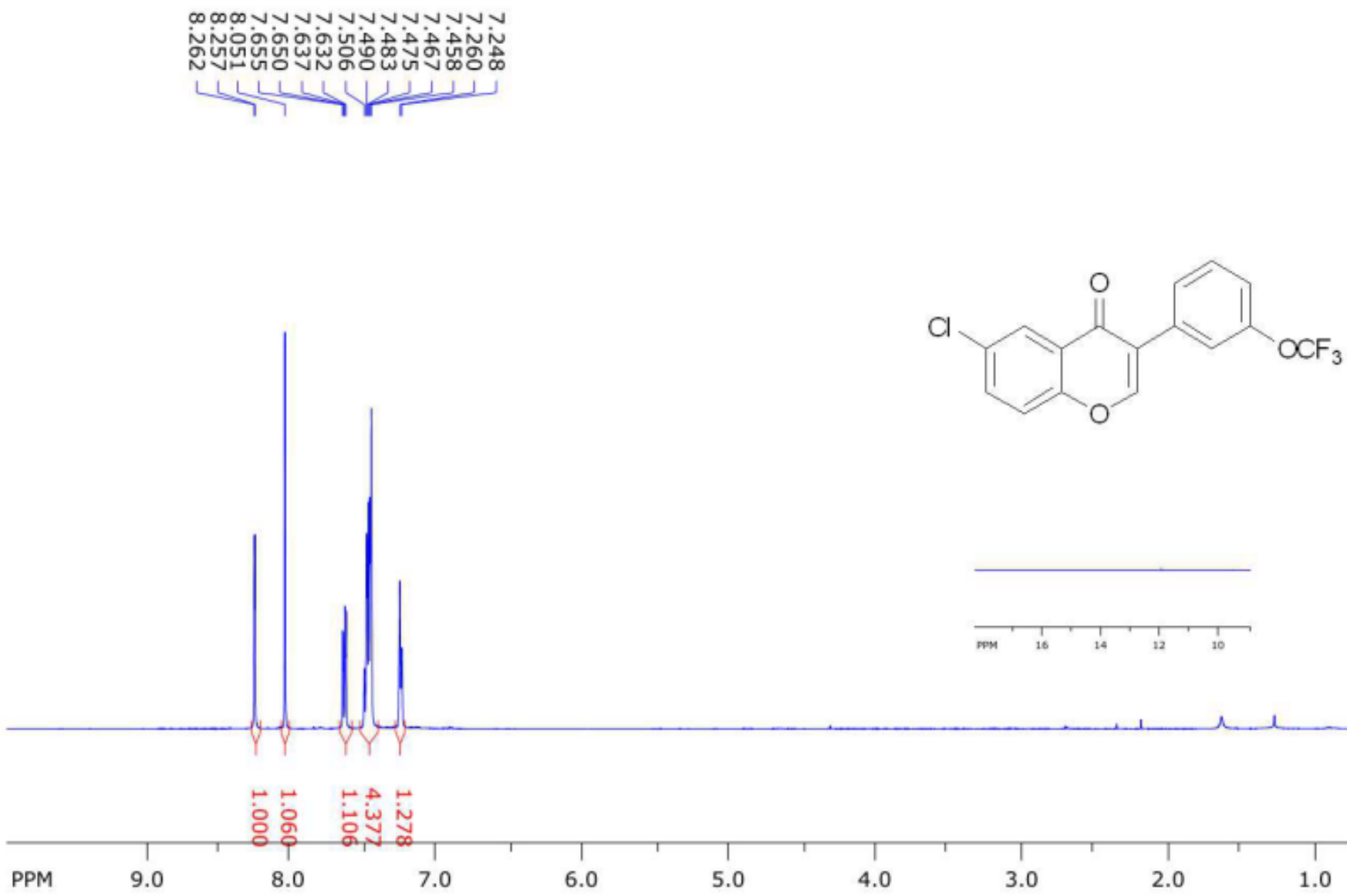

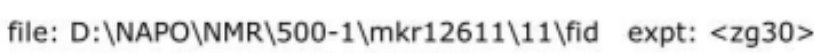
transmitter freq.: $500.133001 \mathrm{MHz}$

time domain size: 65536 points

width: $12335.53 \mathrm{~Hz}=24.6645 \mathrm{ppm}=0.188225 \mathrm{~Hz} / \mathrm{pt}$

number of scans: 24 


\section{Compound 5ah (NMR in $\left.\mathrm{CDCl}_{3}\right)$}

SpinWorks 4: ISM $10113 \mathrm{C}$

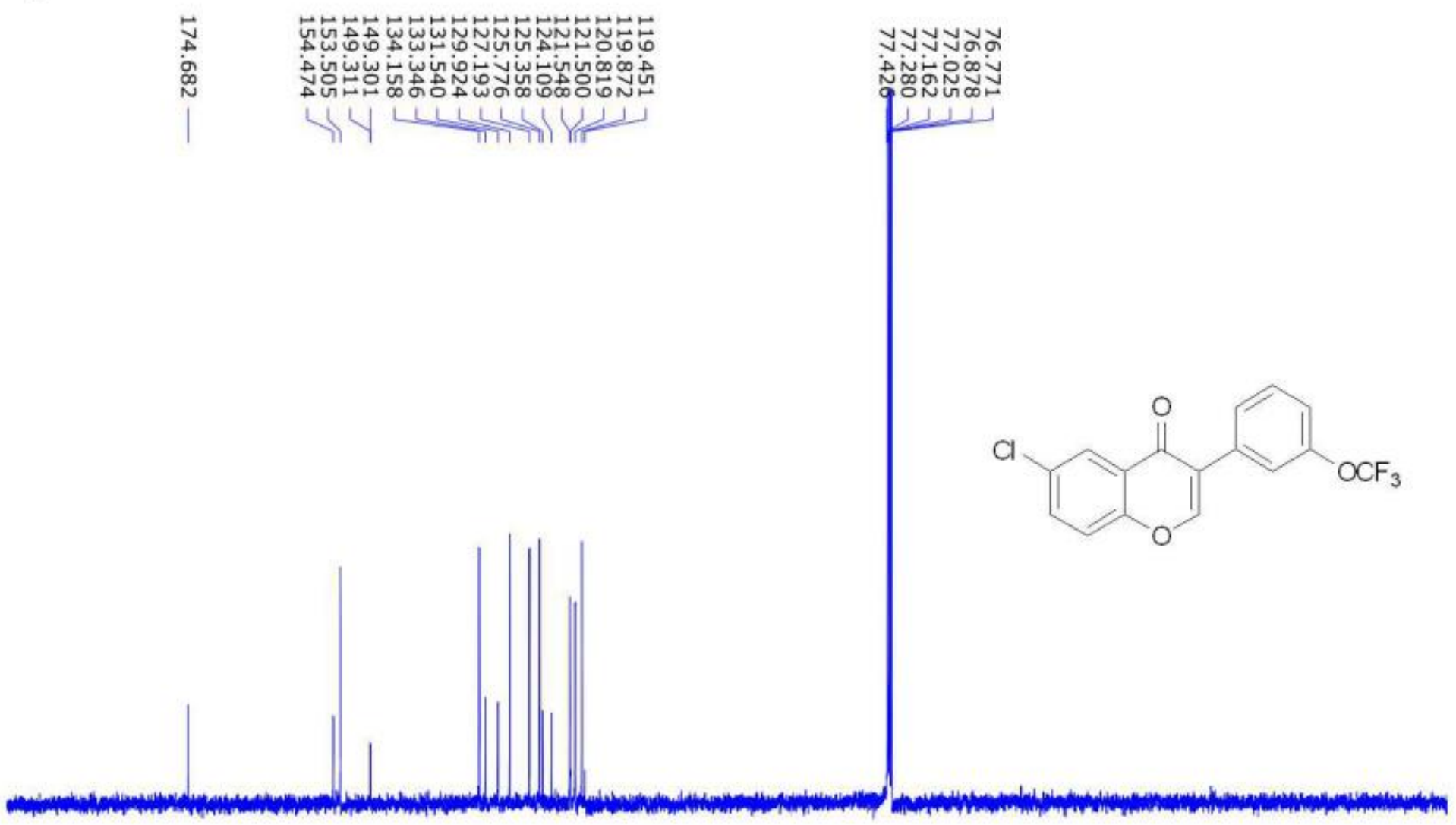

\begin{tabular}{cc|c|c|c|c|c}
\hline & & 1 & & 1 \\
PPM & 180 & 160 & & 140 & 120
\end{tabular}

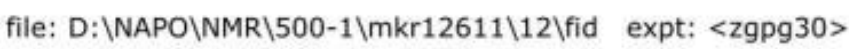

100

80

60

40

20

transmitter freq.: $125.772879 \mathrm{MHz}$

freq. of 0 ppm: $125.757793 \mathrm{MHz}$

time domain size: 65536 points

processed size: 32768 complex points

width: $36057.69 \mathrm{~Hz}=286.6889 \mathrm{ppm}=0.550197 \mathrm{~Hz} / \mathrm{pt}$

LB: 2.000 GF: 0.0000

number of scans: 256 


\section{Compound 5ai (NMR in $\left.\mathrm{CDCl}_{3}\right)$}

SpinWorks 4: ISM $1141 \mathrm{H}$
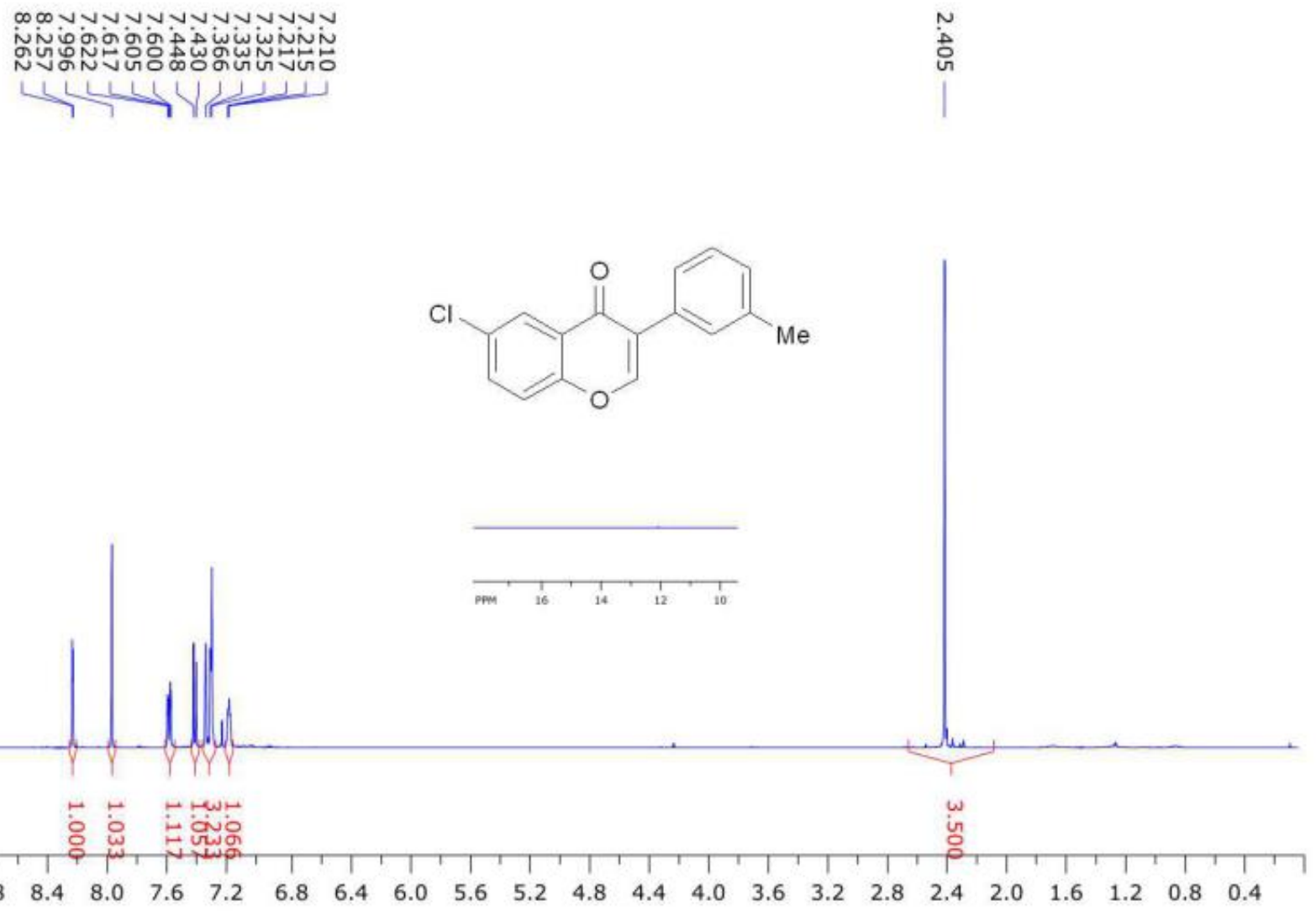

$\begin{array}{llllllllllllllllllllllll}\text { PPM } & 9.2 & 8.8 & 8.4 & 8.0 & 7.6 & 7.2 & 6.8 & 6.4 & 6.0 & 5.6 & 5.2 & 4.8 & 4.4 & 4.0 & 3.6 & 3.2 & 2.8 & 2.4 & 2.0 & 1.6 & 1.2 & 0.8 & 0.4\end{array}$

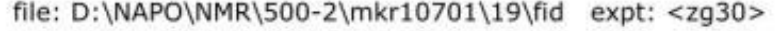
transmitter freq.: $500.133001 \mathrm{MHz}$

time domain size: 65536 points

width: $12335.53 \mathrm{~Hz}=24.6645 \mathrm{ppm}=0.188225 \mathrm{~Hz} / \mathrm{pt}$

number of scans: 24 freq. of 0 ppm: $500.130025 \mathrm{MHz}$

processed size: 65536 complex points

LB: 0.300 GF: 0.0000

$\mathrm{Hz} / \mathrm{cm}: 199.224 \mathrm{ppm} / \mathrm{cm}: 0.39834$ 


\section{Compound 5ai (NMR in $\left.\mathrm{CDCl}_{3}\right)$}

SpinWorks 4: ISM 114 13C
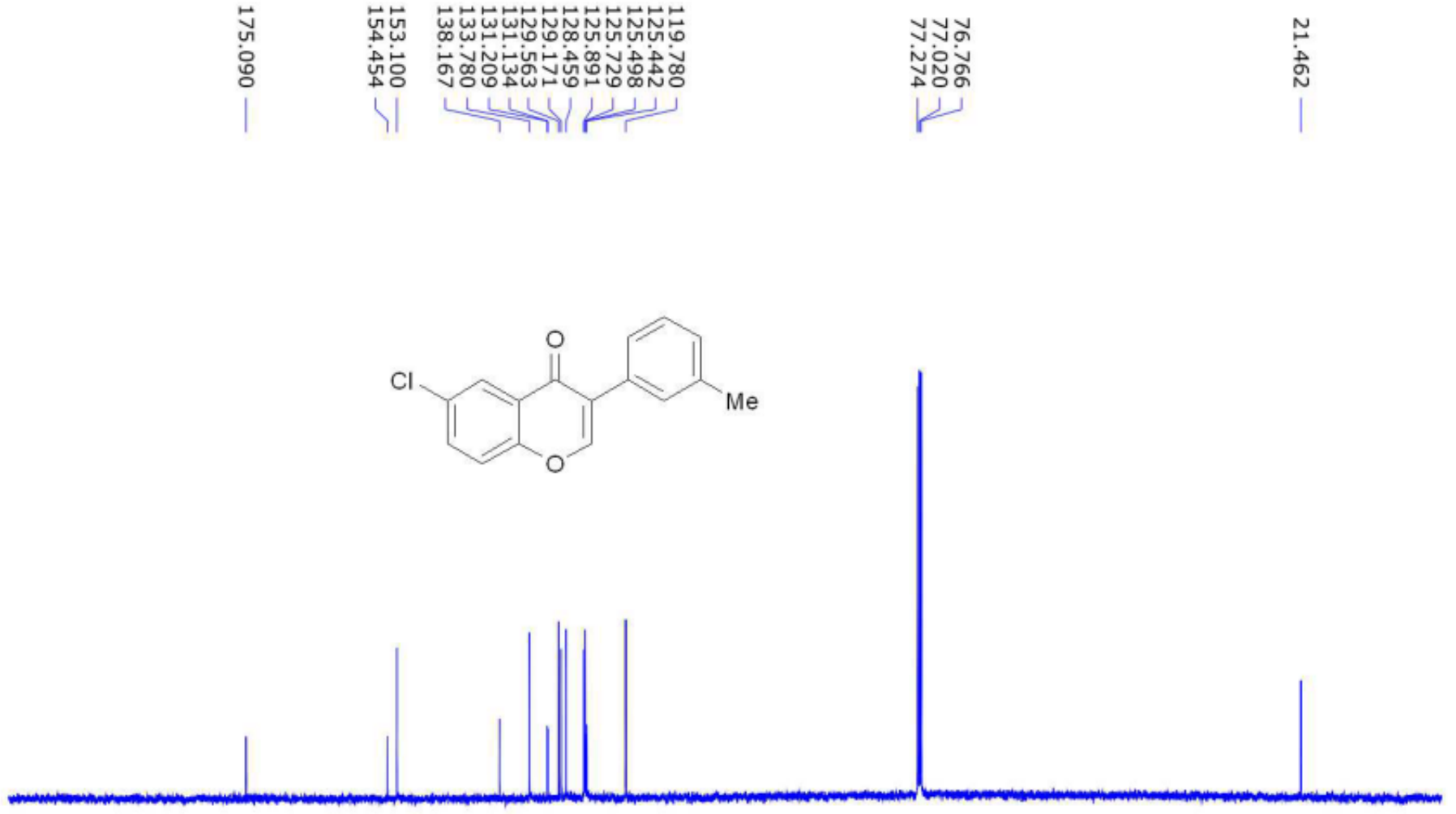

PPM

180

160

140

120

100

80

60

40

20

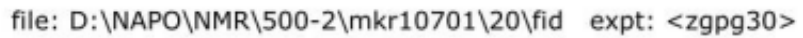
transmitter freq.: $125.772879 \mathrm{MHz}$

time domain size: 65536 points

freq. of $0 \mathrm{ppm}: 125.757798 \mathrm{MHz}$

processed size: 32768 complex points

width: $36057.69 \mathrm{~Hz}=286.6889 \mathrm{ppm}=0.550197 \mathrm{~Hz} / \mathrm{pt}$

number of scans: 512

LB: 2.000 GF: 0.0000

$\mathrm{Hz} / \mathrm{cm}: 1049.821 \quad \mathrm{ppm} / \mathrm{cm}: 8.34696$ 


\section{Compound 5aj (NMR in $\mathrm{CDCl}_{3}$ )}

SpinWorks 4: ISM $1151 \mathrm{H}$

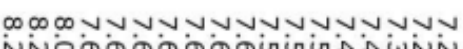

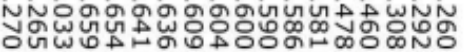

L

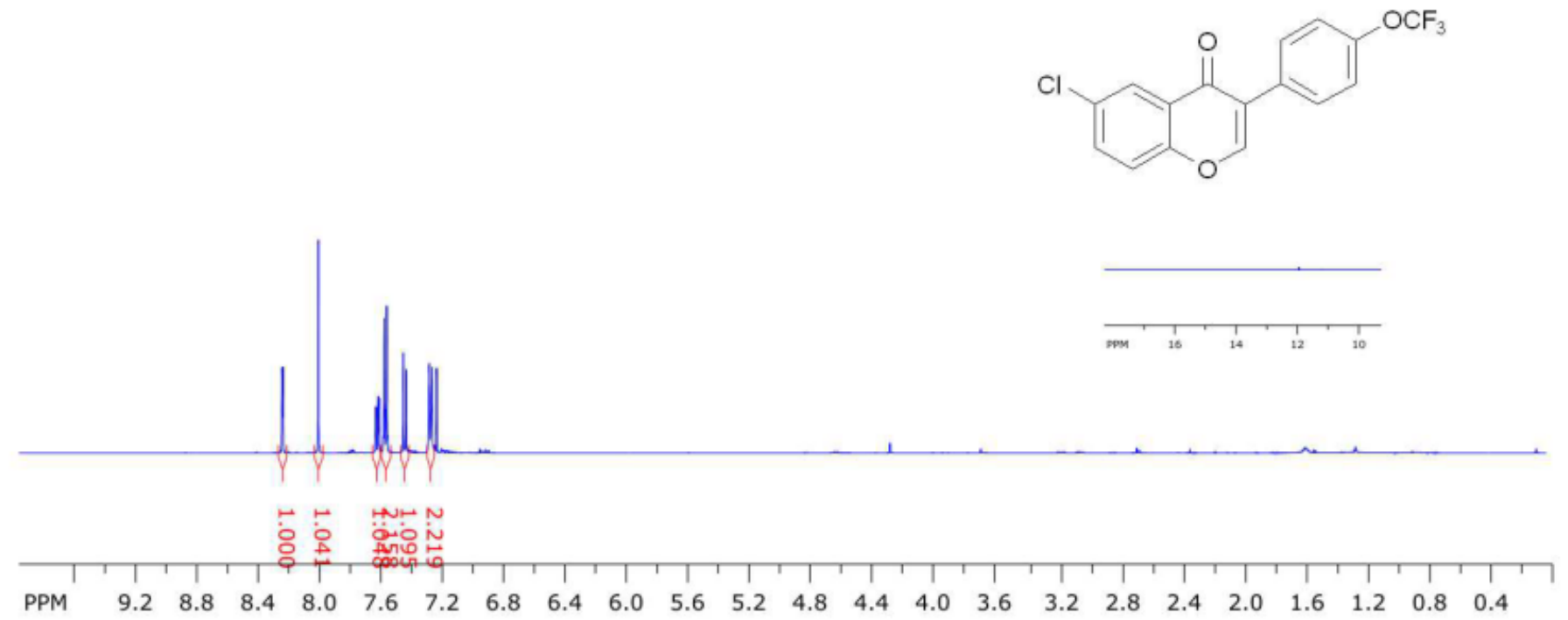

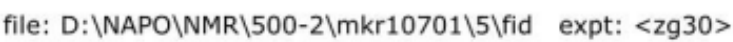
transmitter freq.: $500.133001 \mathrm{MHz}$

time domain size: 65536 points

freq. of 0 ppm: $500.130024 \mathrm{MHz}$

width: $12335.53 \mathrm{~Hz}=24.6645 \mathrm{ppm}=0.188225 \mathrm{~Hz} / \mathrm{pt}$

processed size: 65536 complex points

LB: 0.300 GF: 0.0000

$\mathrm{Hz} / \mathrm{cm}: 199.770 \mathrm{ppm} / \mathrm{cm}: 0.39943$

number of scans: 24 


\section{Compound 5aj (NMR in $\mathrm{CDCl}_{3}$ )}

SpinWorks 4: ISM 115-1 13C
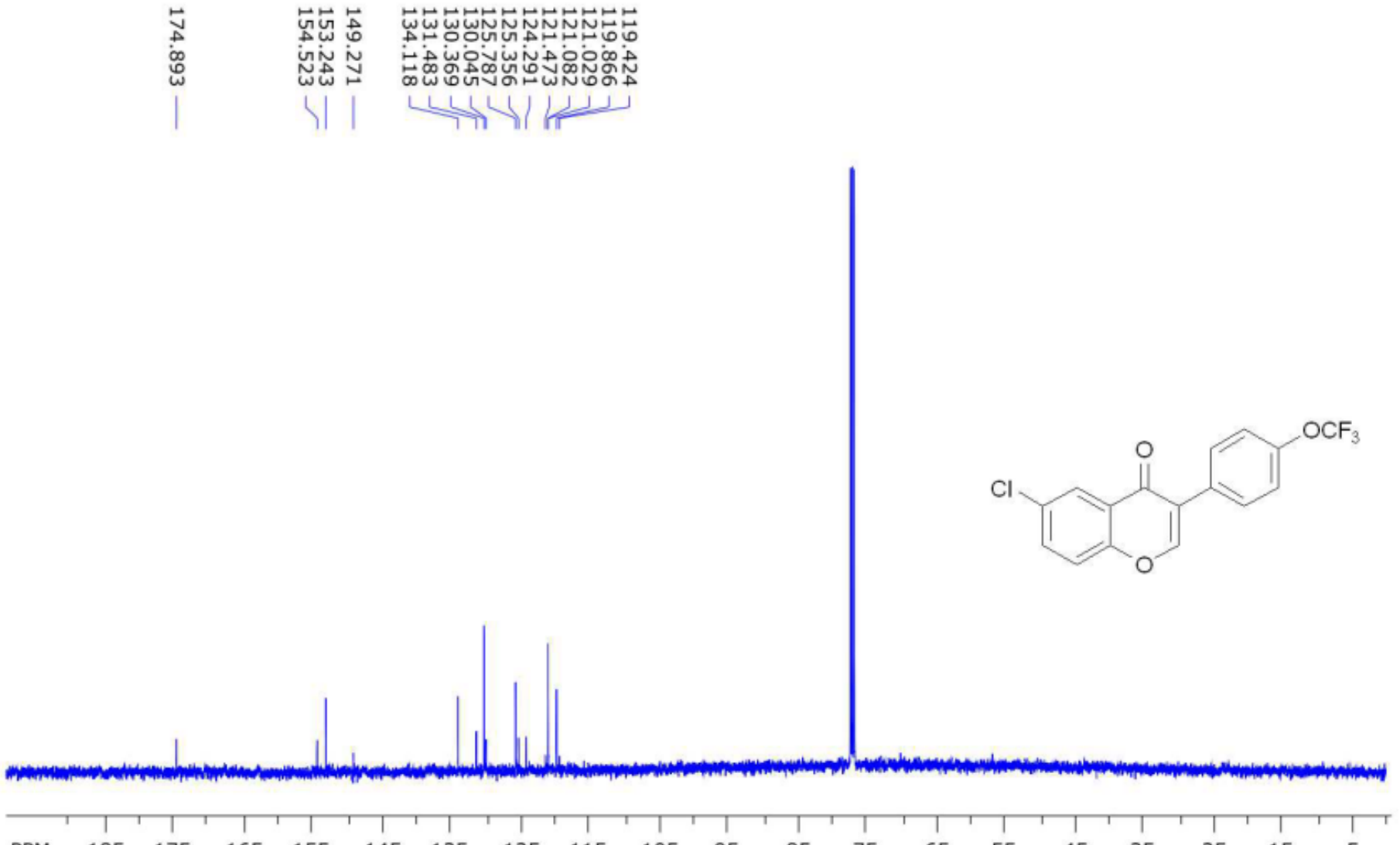

freq. of 0 ppm: $125.757792 \mathrm{MHz}$

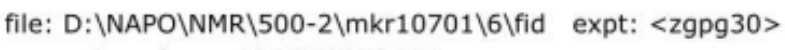
transmitter freq.: $125.772879 \mathrm{MHz}$

processed size: 32768 complex points

time domain size: 65536 points

LB: 2.000 GF: 0.0000

width: $36057.69 \mathrm{~Hz}=286.6889 \mathrm{ppm}=0.550197 \mathrm{~Hz} / \mathrm{pt}$

$\mathrm{Hz} / \mathrm{cm}: 1005.148 \mathrm{ppm} / \mathrm{cm}: 7.99177$ 


\section{Compound 5ak (NMR in $\mathrm{CDCl}_{3}$ )}

SpinWorks 4: ISM $2491 \mathrm{H}$

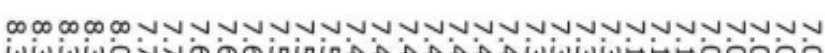

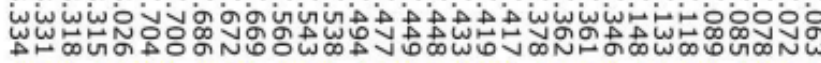

1. ता गा1
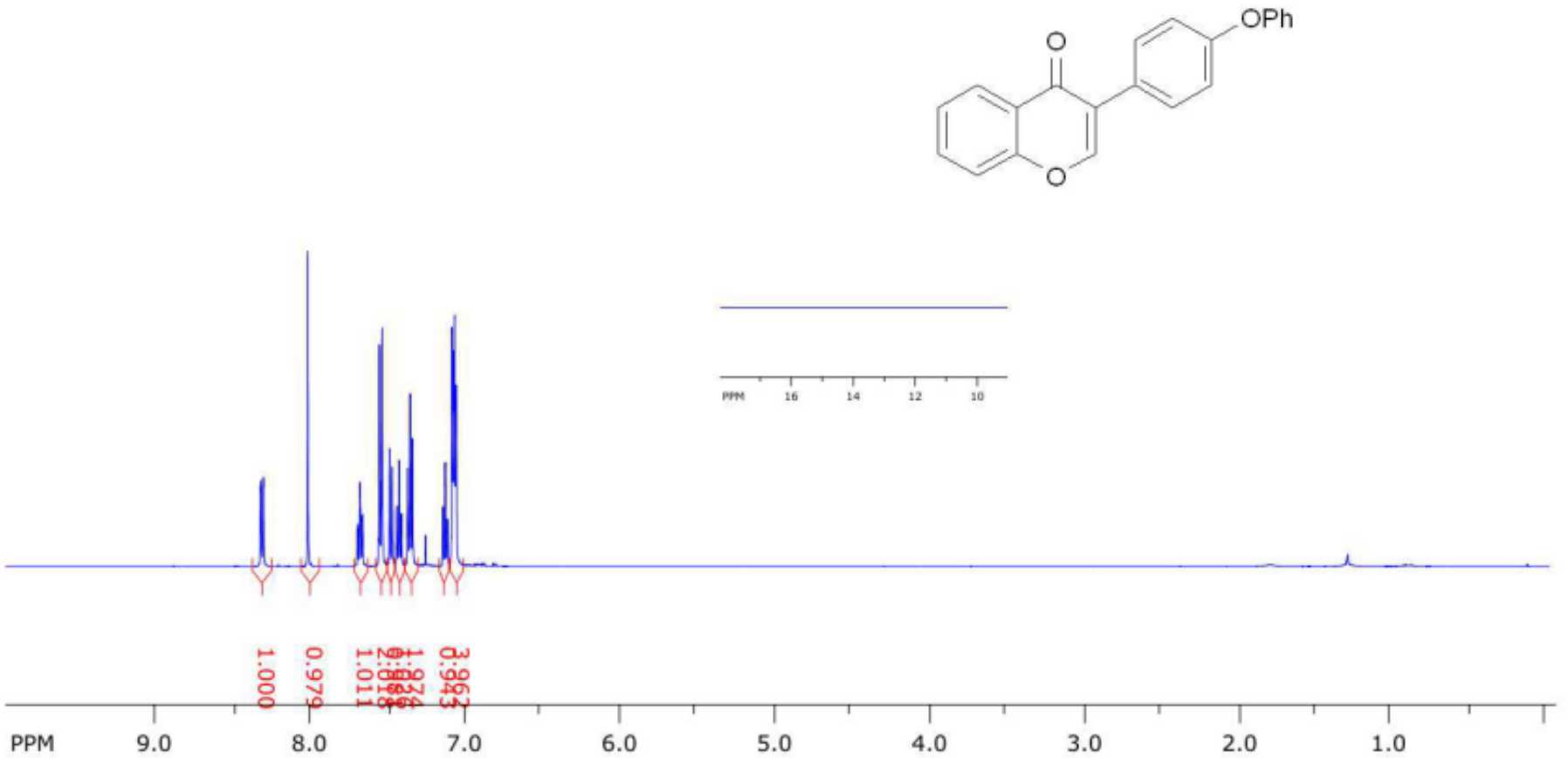

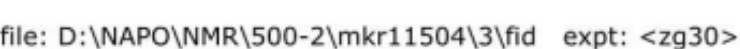

freq. of 0 ppm: $500.130023 \mathrm{MHz}$

transmitter freq.: $500.133001 \mathrm{MHz}$

time domain size: 65536 points

processed size: 65536 complex points

width: $12335.53 \mathrm{~Hz}=24.6645 \mathrm{ppm}=0.188225 \mathrm{~Hz} / \mathrm{pt}$

LB: 0.300 GF: 0.0000

$\mathrm{Hz} / \mathrm{cm}: 200.862 \mathrm{ppm} / \mathrm{cm}: 0.40162$

number of scans: 24 


\section{Compound 5ak (NMR in $\mathrm{CDCl}_{3}$ )}

SpinWorks 4: ISM $24913 \mathrm{C}$

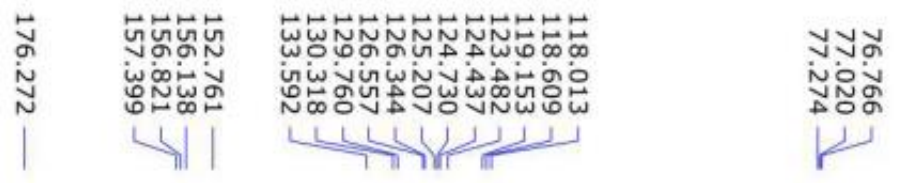

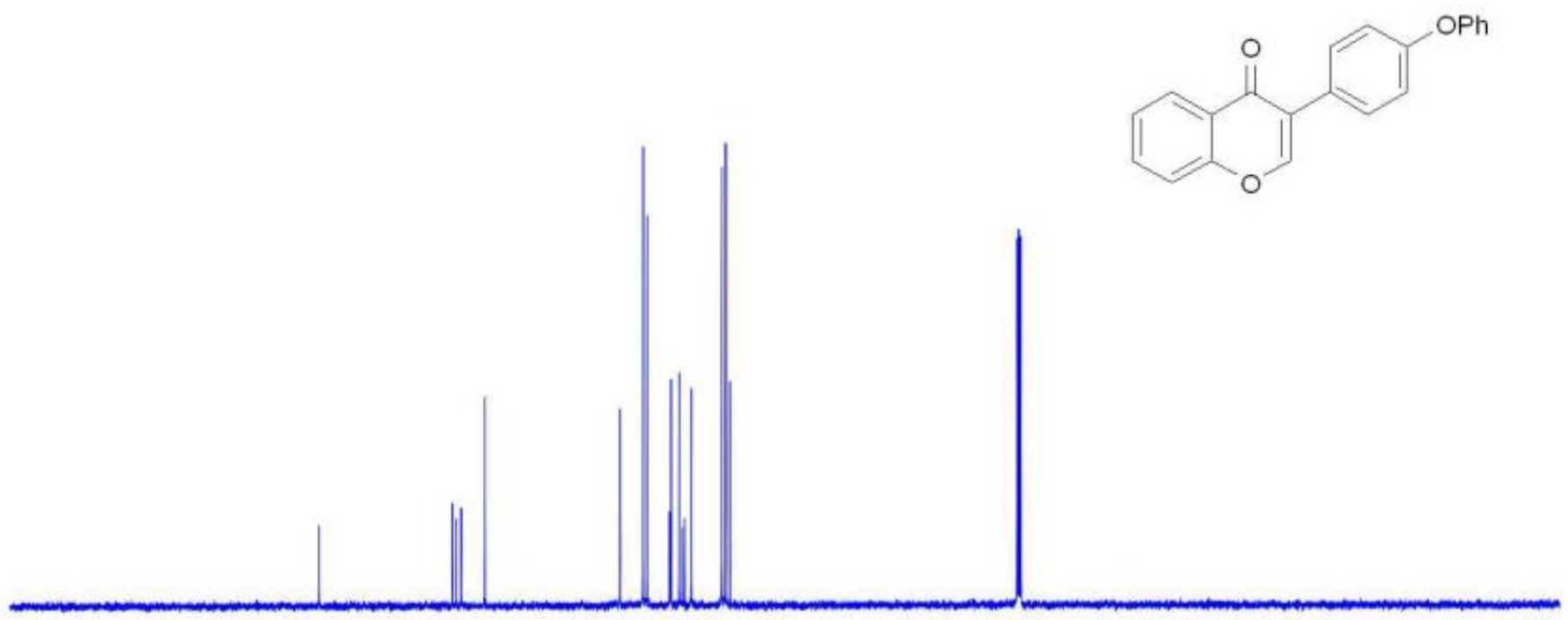

\begin{tabular}{cc|c|c} 
& & & \\
PPM & 200 & 1 \\
& & 180
\end{tabular}

160

140

120

100

80

60

40

20

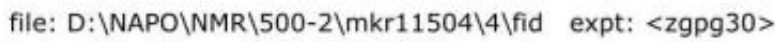

transmitter freq.: $125.772879 \mathrm{MHz}$

freq. of $0 \mathrm{ppm}: 125.757800 \mathrm{MHz}$

time domain size: 65536 points

processed size: 32768 complex points

LB: 2.000 GF: 0.0000

$\mathrm{Hz} / \mathrm{cm}: 1105.663 \mathrm{ppm} / \mathrm{cm}: 8.79095$

number of scans: 512 


\section{Compound 5al (NMR in $\left.\mathrm{CDCl}_{3}\right)$}

SpinWorks 4: ISM 95-1 1H
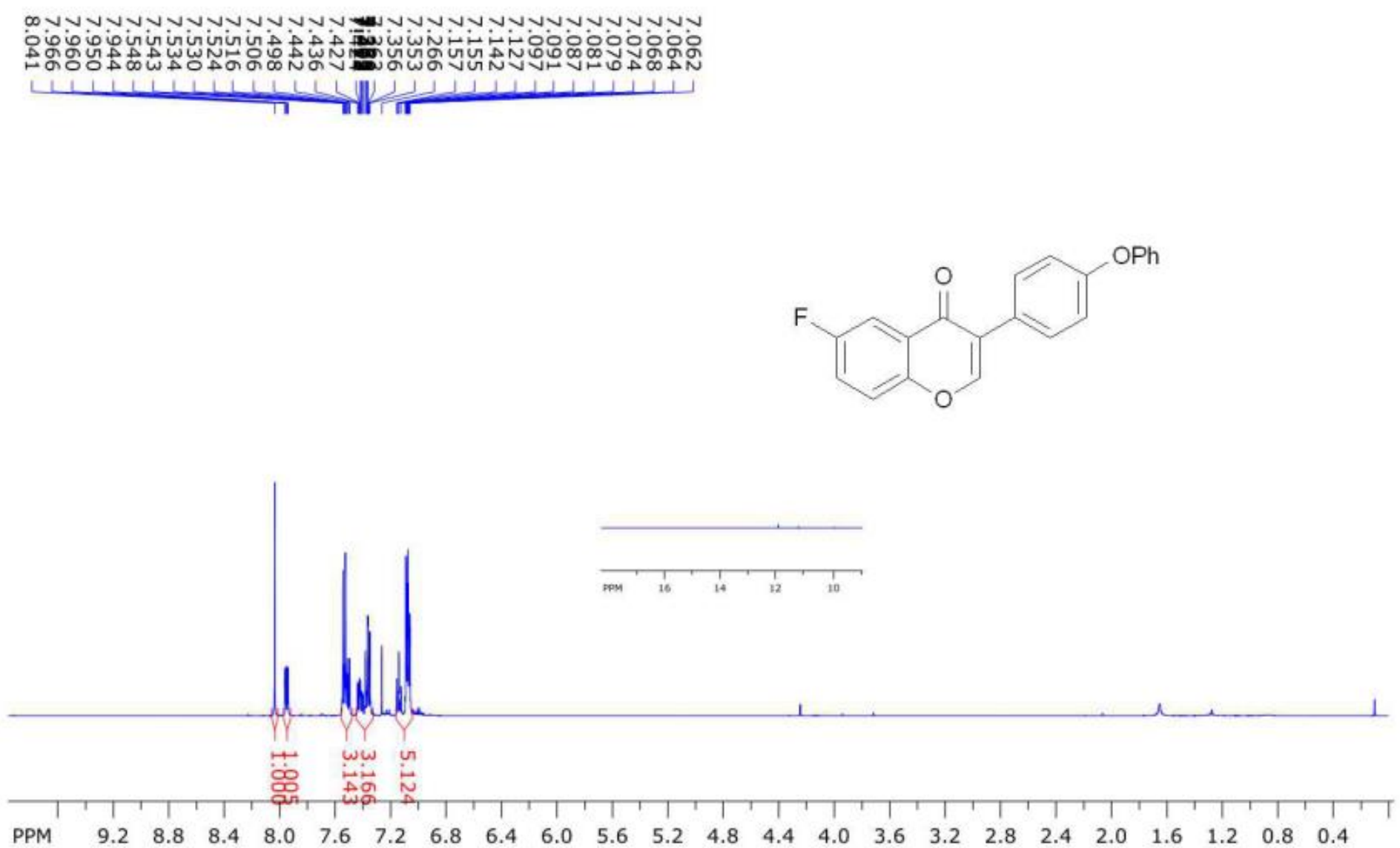

file: D: \NAPO\NMR $500-1 \backslash m k r 11611 \backslash 37 \backslash$ fid expt: <zg30> transmitter freq.: $500.133001 \mathrm{MHz}$

time domain size: 65536 points

width: $12335.53 \mathrm{~Hz}=24.6645 \mathrm{ppm}=0.188225 \mathrm{~Hz} / \mathrm{pt}$

number of scans: 16 freq. of 0 ppm: $500.130020 \mathrm{MHz}$

processed size: 65536 complex points

LB: 0.300 GF: 0.0000

$\mathrm{Hz} / \mathrm{cm}: 199.770 \mathrm{ppm} / \mathrm{cm}: 0.39943$ 


\section{Compound 5al (NMR in $\mathrm{CDCl}_{3}$ )}

SpinWorks 4: ISM 95-1 13C
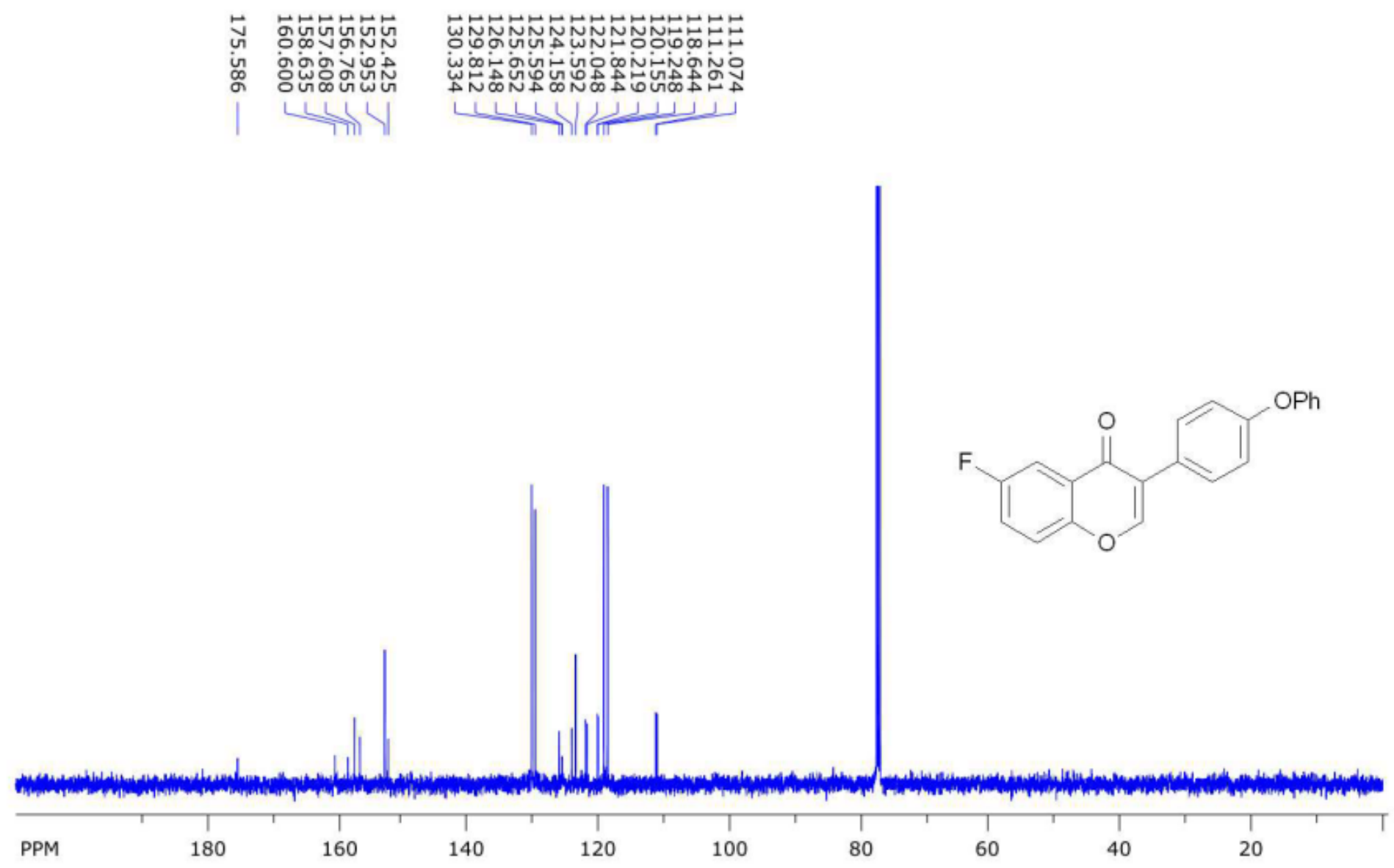

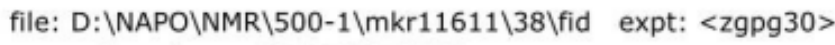
transmitter freq.: $125.772879 \mathrm{MHz}$

time domain size: 65536 points

freq. of 0 ppm: $125.757794 \mathrm{MHz}$

processed size: 32768 complex points

width: $36057.69 \mathrm{~Hz}=286.6889 \mathrm{ppm}=0.550197 \mathrm{~Hz} / \mathrm{pt}$

LB: 2.000 GF: 0.0000

number of scans: 128 


\section{Compound 5am (NMR in $\left.\mathrm{CDCl}_{3}\right)$}

SpinWorks 4: ISM $931 \mathrm{H}$
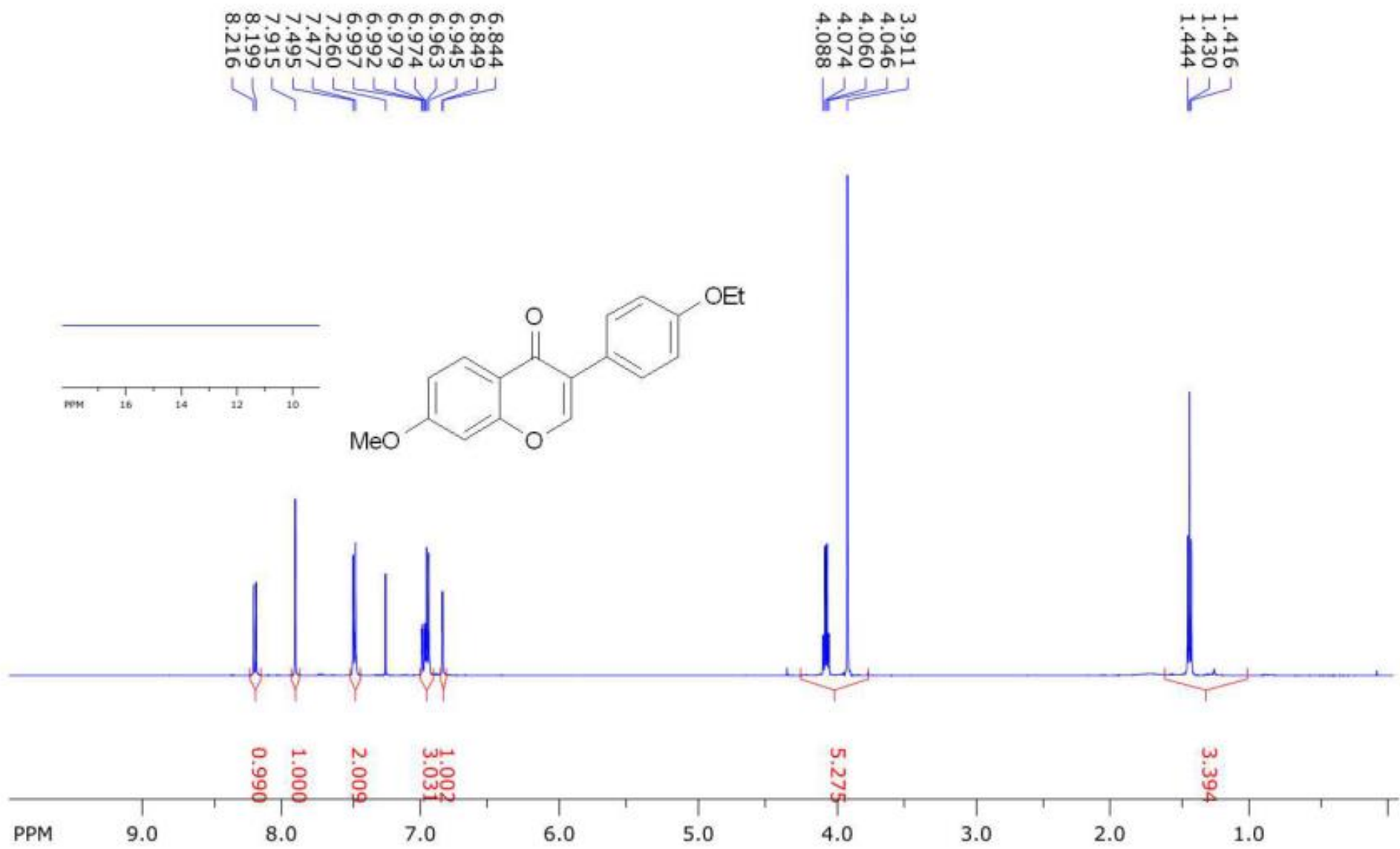

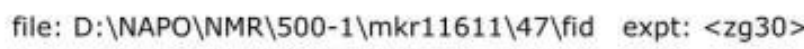
transmitter freq.: $500.133001 \mathrm{MHz}$

time domain size: 65536 points

width: $12335.53 \mathrm{~Hz}=24.6645 \mathrm{ppm}=0.188225 \mathrm{~Hz} / \mathrm{pt}$

freq. of 0 ppm: $500.130023 \mathrm{MHz}$

processed size: 65536 complex points

LB: 0.300 GF: 0.0000

$\mathrm{Hz} / \mathrm{cm}: 200.862 \mathrm{ppm} / \mathrm{cm}: 0.40162$

number of scans: 12 


\section{Compound 5am (NMR in $\left.\mathrm{CDCl}_{3}\right)$}

SpinWorks 4: ISM $9313 \mathrm{C}$
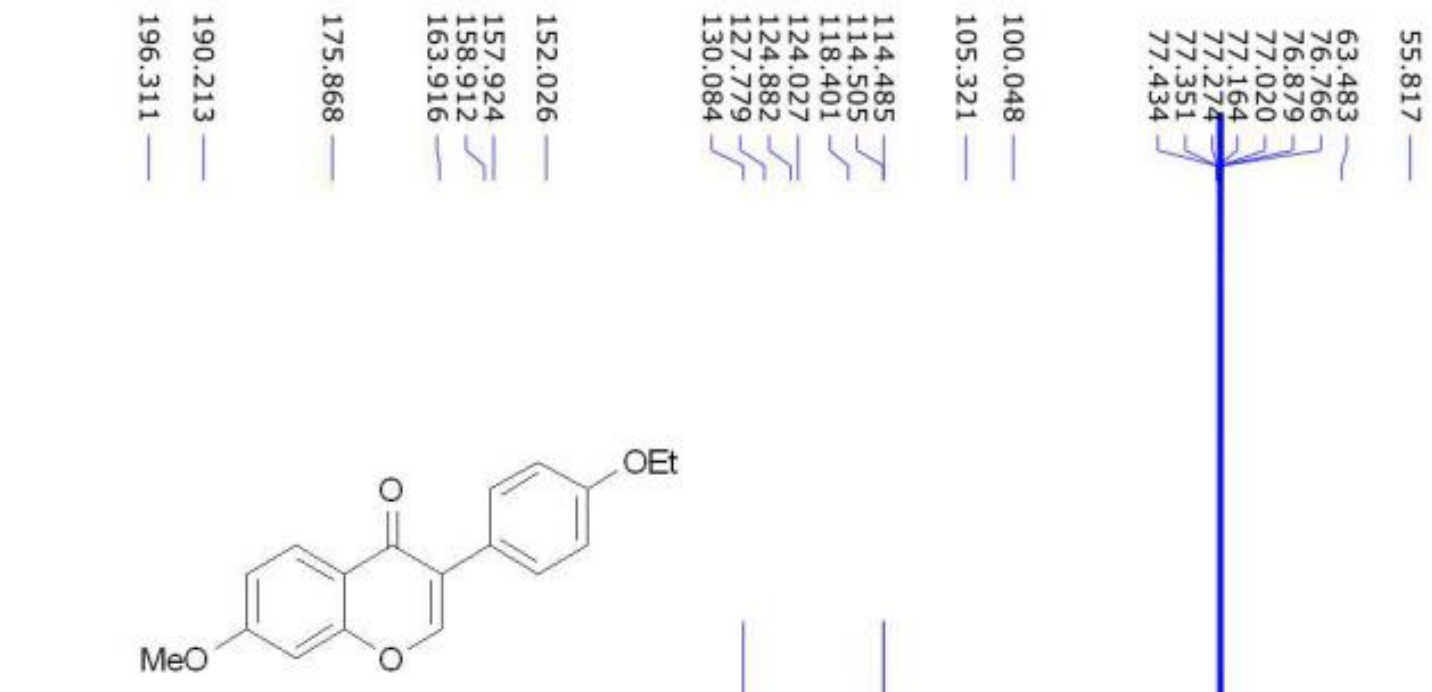

$$
\text { Et }
$$
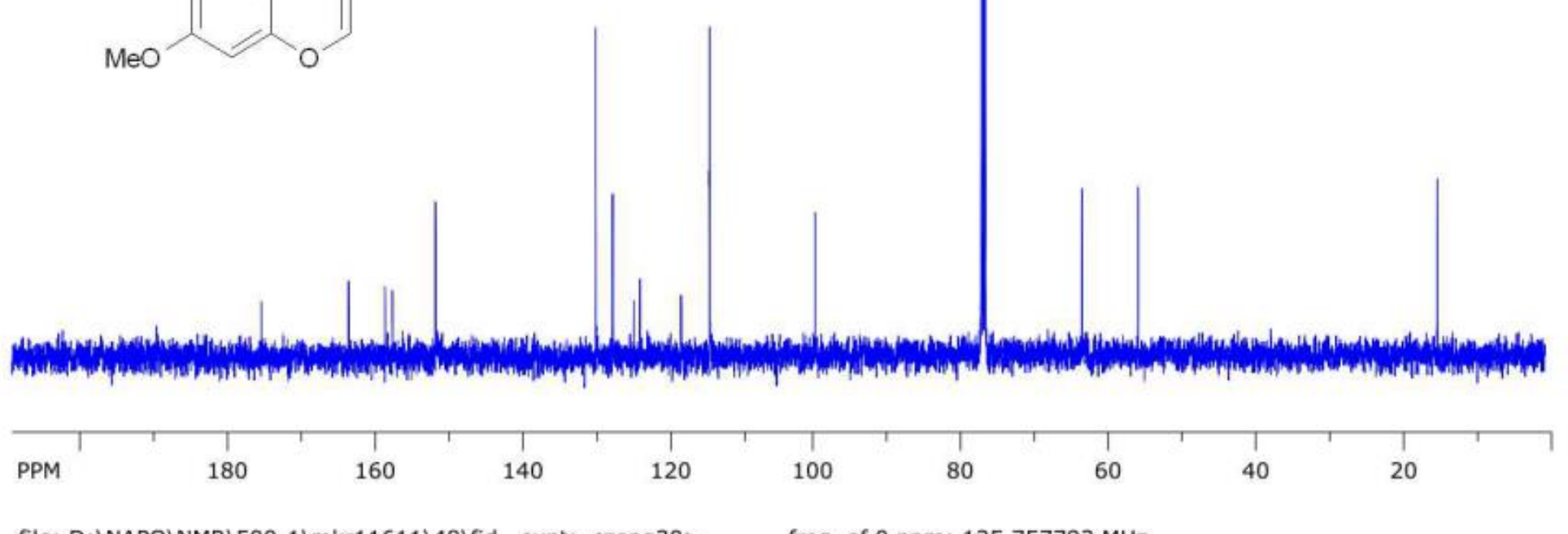

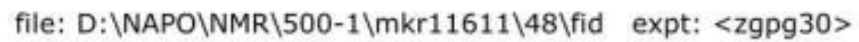
transmitter freq.: $125.772879 \mathrm{MHz}$

time domain size: 65536 points

width: $36057.69 \mathrm{~Hz}=286.6889 \mathrm{ppm}=0.550197 \mathrm{~Hz} / \mathrm{pt}$ number of scans: 128
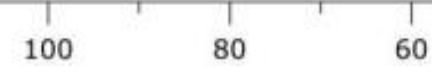

freq. of $0 \mathrm{ppm}: 125.757793 \mathrm{MHz}$ processed size: 32768 complex points LB: 2.000 GF: 0.0000

$\mathrm{Hz} / \mathrm{cm}: 1056.203 \mathrm{ppm} / \mathrm{cm}: 8.39770$ 


\section{Compound 5an (NMR in $\left.\mathrm{CDCl}_{3}\right)$}

SpinWorks 4: ISM 304 1H
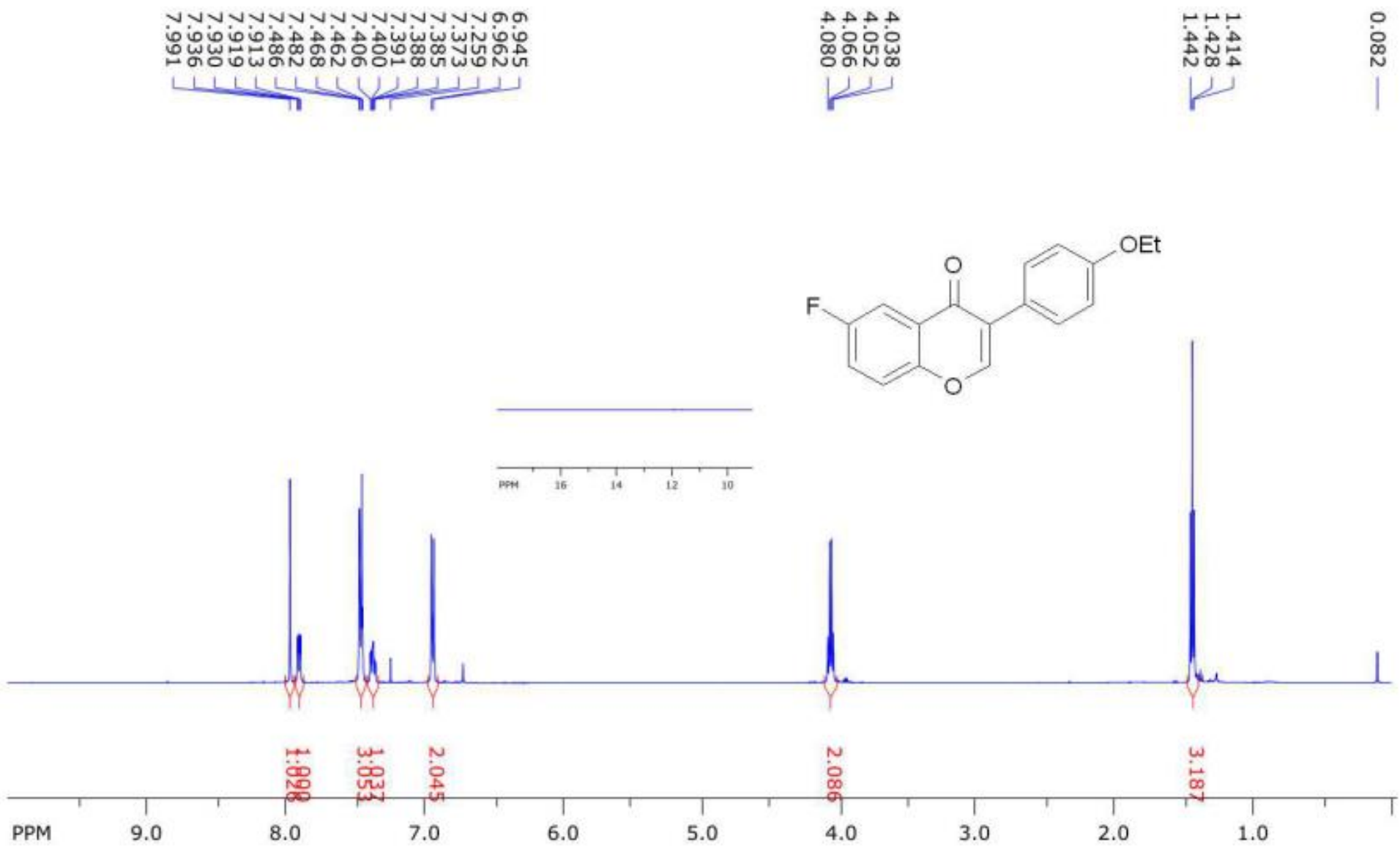

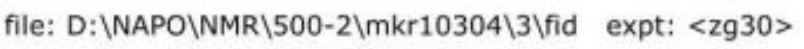
transmitter freq.: $500.133001 \mathrm{MHz}$

time domain size: 65536 points

freq. of 0 ppm: $500.130024 \mathrm{MHz}$

processed size: 65536 complex points

width: $12335.53 \mathrm{~Hz}=24.6645 \mathrm{ppm}=0.188225 \mathrm{~Hz} / \mathrm{pt}$

LB: 0.300 GF: 0.0000

number of scans: 24 


\section{Compound 5an (NMR in $\mathrm{CDCl}_{3}$ )}

SpinWorks 4: ISM 304 13C
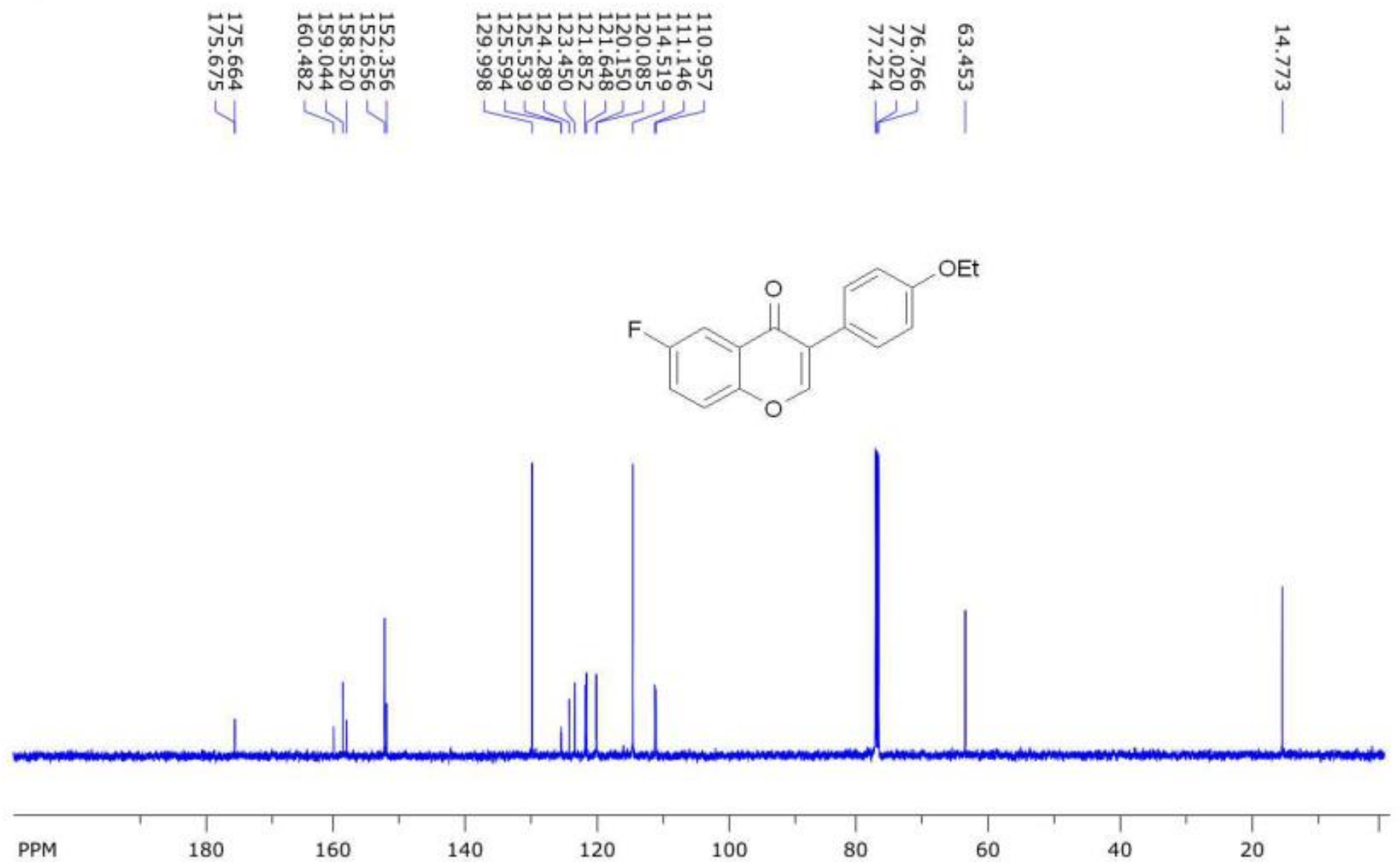

file: D:\NAPO\NMR $500-2 \backslash m k r 10304 \backslash 4 \backslash$ fid expt: <zgpg30> transmitter freq.: $125.772879 \mathrm{MHz}$

time domain size: 65536 points

freq. of $0 \mathrm{ppm}: 125.757799 \mathrm{MHz}$

width: $36057.69 \mathrm{~Hz}=286.6889 \mathrm{ppm}=0.550197 \mathrm{~Hz} / \mathrm{pt}$ processed size: 32768 complex points

LB: 2.000 GF: 0.0000 number of scans: 164

$\mathrm{Hz} / \mathrm{cm}: 1059.394 \mathrm{ppm} / \mathrm{cm}: 8.42307$ 


\section{Compound 5ao (NMR in $\mathrm{CDCl}_{3}$ )}

SpinWorks 4: ISM $1241 \mathrm{H}$

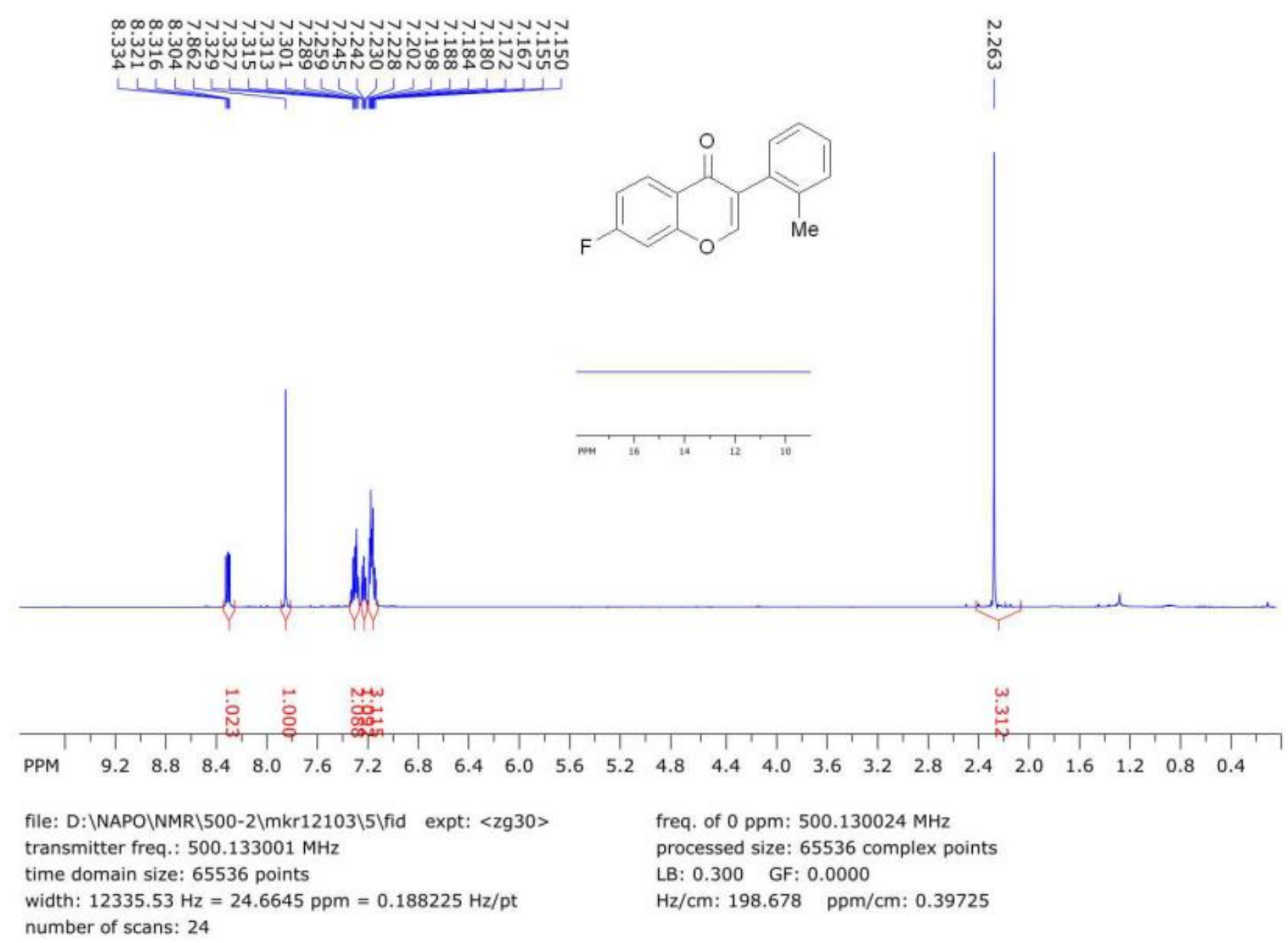




\section{Compound 5ao (NMR in $\mathrm{CDCl}_{3}$ )}

SpinWorks 4: ISM 124 13C

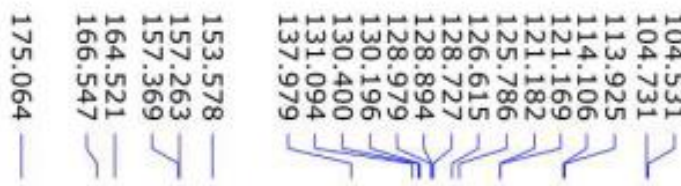

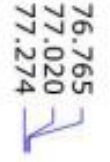
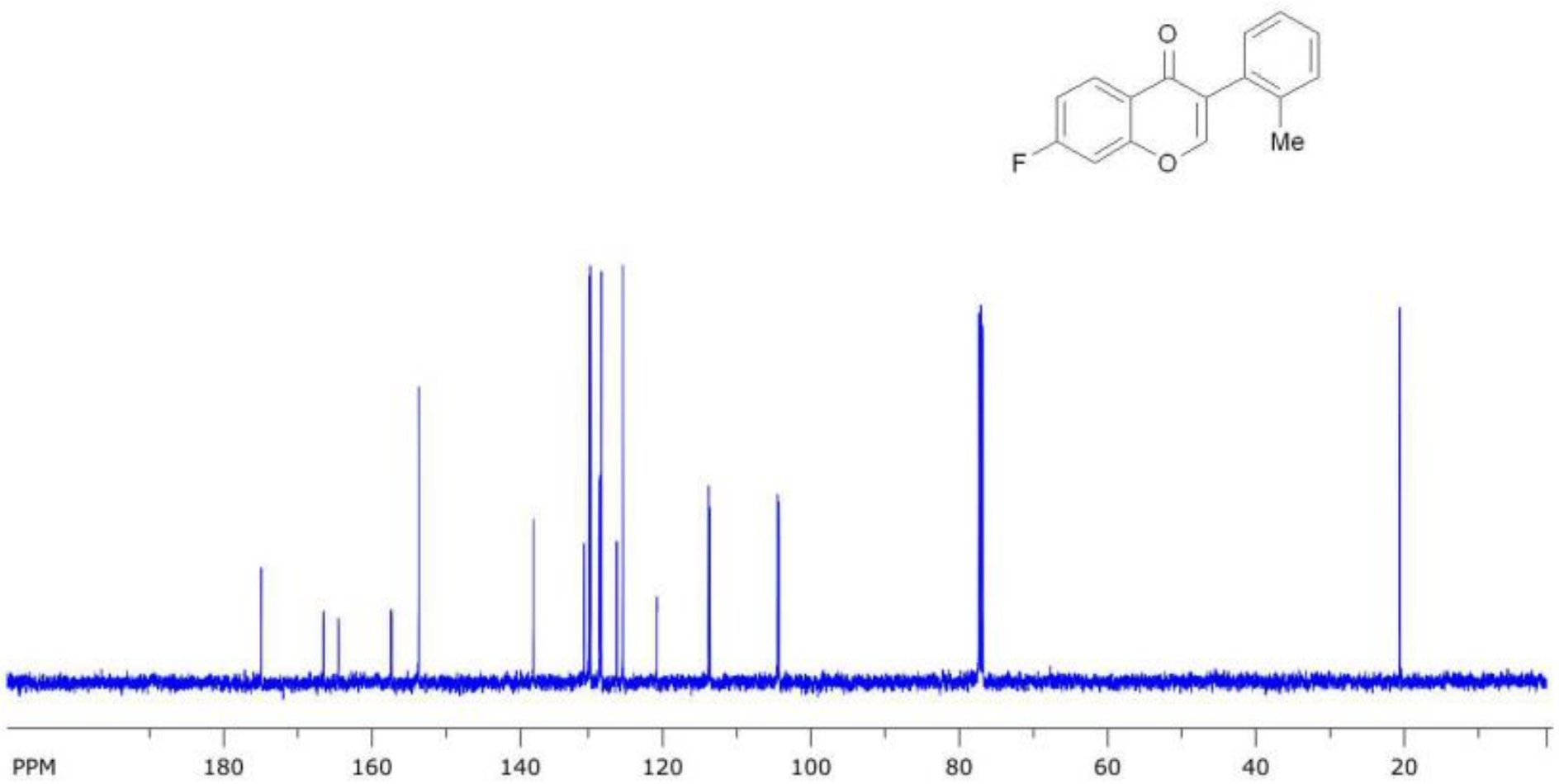

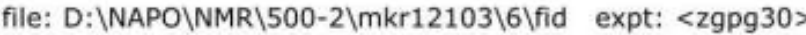
transmitter freq.: $125.772879 \mathrm{MHz}$

freq. of 0 ppm: $125.757802 \mathrm{MHz}$

processed size: 32768 complex points

LB: 2.000 GF: 0.0000

$\mathrm{Hz} / \mathrm{cm}: 1054.608 \quad \mathrm{ppm} / \mathrm{cm}: 8.38502$

width: $36057.69 \mathrm{~Hz}=286.6889 \mathrm{ppm}=0.550197 \mathrm{~Hz} / \mathrm{pt}$

number of scans: 128 


\section{Compound 5ap (NMR in $\mathrm{CDCl}_{3}$ )}

SpinWorks 4: ISM 99-1 1H
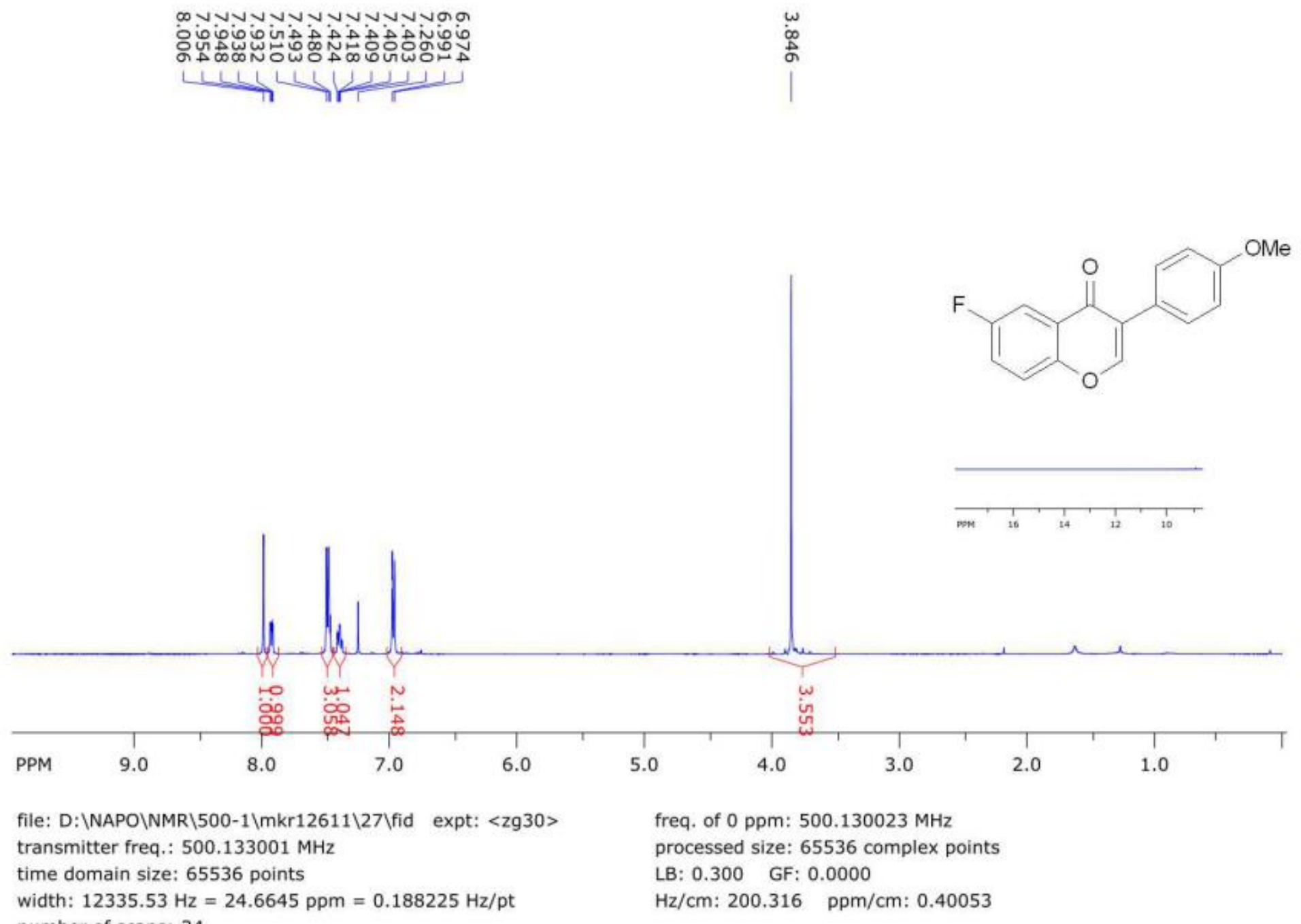

number of scans: 24 


\section{Compound 5ap (NMR in $\left.\mathrm{CDCl}_{3}\right)$}

SpinWorks 4: ISM 99-1 13C

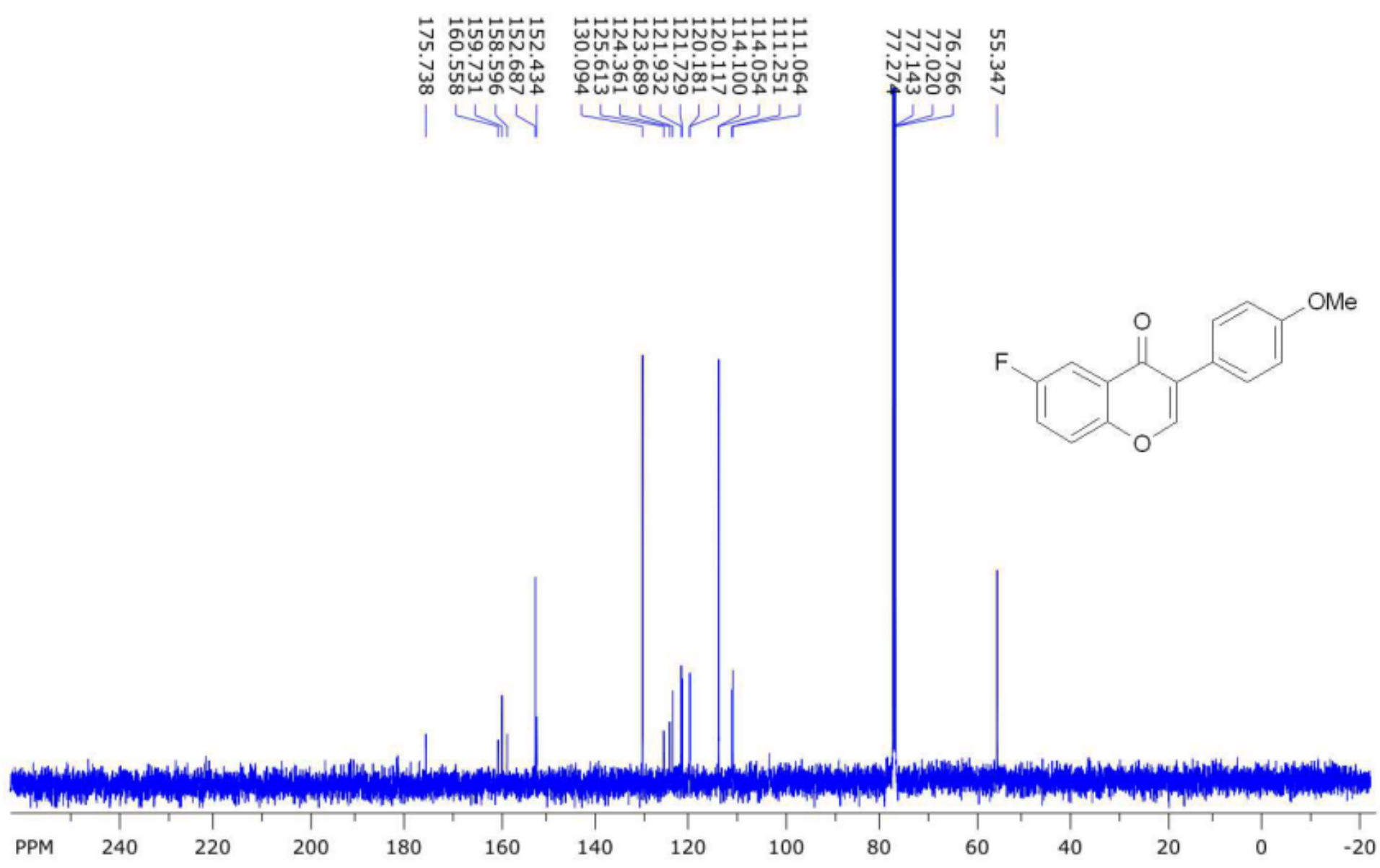

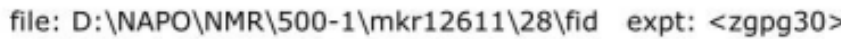
transmitter freq.: $125.772879 \mathrm{MHz}$

time domain size: 65536 points

freq. of $0 \mathrm{ppm}: 125.757793 \mathrm{MHz}$

width: $36057.69 \mathrm{~Hz}=286.6889 \mathrm{ppm}=0.550197 \mathrm{~Hz} / \mathrm{pt}$ processed size: 32768 complex points

LB: 2.000 GF: 0.0000 number of scans: 256

$\mathrm{Hz} / \mathrm{cm}: 1442.308 \mathrm{ppm} / \mathrm{cm}: 11.46756$ 